\title{
BROOKHGUEN
}

NATIONAL LABORATORY

BNL-77973-2007-IR

\section{Report of the US long baseline neutrino experiment study}

\author{
V. Barger, et al.
}

May, 2007

\author{
Physics Department \\ Electronic Detector Group \\ Brookhaven National Laboratory \\ P.O. Box 5000 \\ Upton, NY 11973-5000 \\ www.bnl.gov
}

\begin{abstract}
Notice: This manuscript has been authored by employees of Brookhaven Science Associates, LLC under Contract No. DE-AC02-98CH10886 with the U.S. Department of Energy. The publisher by accepting the manuscript for publication acknowledges that the United States Government retains a non-exclusive, paid-up, irrevocable, world-wide license to publish or reproduce the published form of this manuscript, or allow others to do so, for United States Government purposes.
\end{abstract}




\section{DISCLAIMER}

This report was prepared as an account of work sponsored by an agency of the United States Government. Neither the United States Government nor any agency thereof, nor any of their employees, nor any of their contractors, subcontractors, or their employees, makes any warranty, express or implied, or assumes any legal liability or responsibility for the accuracy, completeness, or any third party's use or the results of such use of any information, apparatus, product, or process disclosed, or represents that its use would not infringe privately owned rights. Reference herein to any specific commercial product, process, or service by trade name, trademark, manufacturer, or otherwise, does not necessarily constitute or imply its endorsement, recommendation, or favoring by the United States Government or any agency thereof or its contractors or subcontractors. The views and opinions of authors expressed herein do not necessarily state or reflect those of the United States Government or any agency thereof. 


\section{Report of the US long baseline neutrino experiment study}

D. Bogert, D. Finley, C. Laughton, S. Pordes, A. Marchionni, R. Rameika, N. Saoulidou, R. Zwaska, ${ }^{1}$ M. Bishai, M. Diwan, M. Dierckxsens, H. Kirk, S. Kahn, N. Simos, W. Marciano, Z. Parsa, B. Viren, ${ }^{2}$ C. Lewis, ${ }^{3}$ C. Bromberg, ${ }^{4}$ B. T. Fleming, A. Curioni, ${ }^{5}$ C. K. Jung, C. Yanagisawa, ${ }^{6}$ F. Dufour, E. Kearns, ${ }^{7}$ T. Kirk, E. D. Zimmerman, ${ }^{8}$ R. Wilson, ${ }^{9}$ K. McDonald, ${ }^{10}$ P. Huber, V. Barger, ${ }^{11}$ W. Winter, ${ }^{12}$ K. Whisnant, ${ }^{13}$ D. Marfatia, ${ }^{14}$ K. Lande, A. K. Mann, R. Van Berg, ${ }^{15}$ K. Lesko, W. Y. Lee, ${ }^{16}$ M. Messier, ${ }^{17}$ K. Whisnant, ${ }^{13}$ W. Pariseau, ${ }^{18}$ P. Litchfield, M. Marshak, ${ }^{19}$ and R. Potenza ${ }^{20}$

${ }^{1}$ Fermi National Accelerator Laboratory

${ }^{2}$ Brookhaven National Laboratory

${ }^{3}$ Columbia University

${ }^{4}$ Michigan State University

${ }^{5}$ Yale University

${ }^{6}$ State University of New York, Stonybrook

${ }^{7}$ Boston University

${ }^{8}$ University of Colorado

${ }^{9}$ Colorado State University

${ }^{10}$ Princeton University

${ }^{11}$ University of Wisconsin

${ }^{12}$ University of Würzburg

${ }^{13}$ Iowa State University

${ }^{14}$ University of Kansas

${ }^{15}$ University of Pennsylvania

${ }^{16}$ Lawrence Berkeley National Laboratory

${ }^{17}$ Indiana University

${ }^{18}$ University of Utah

${ }^{19}$ University of Minnesota

${ }^{20}$ University Di Catania

(Dated: May 4, 2007) 


\begin{abstract}
This report provides the results of an extensive and important study of the potential for a U.S. scientific program that will extend our knowledge of neutrino oscillations well beyond what can be anticipated from ongoing and planned experiments worldwide. The program examined here has the potential to provide the U.S. particle physics community with world leading experimental capability in this intensely interesting and active field of fundamental research. Furthermore, this capability is not likely to be challenged anywhere else in the world for at least two decades into the future. The present study was initially commissioned in April 2006 by top research officers of Brookhaven National Laboratory and Fermilab and, as the study evolved, it also provides responses to questions formulated and addressed to the study group by the Neutrino Scientific Advisory Committee (NuSAG) of the U.S. DOE and NSF. The participants in the study, its Charge and history, plus the study results and conclusions are provided in this report and its appendices. A summary of the conclusions is provided in the Executive Summary.
\end{abstract}




\section{Contents}

1. Executive Summary $\quad 5$

$\begin{array}{lr}\text { 2. Introduction } & 8\end{array}$

3. Physics goals of a Phase-II program 9

4. Strategies for the Phase-II program using a conventional beam 12

5. Accelerator Requirements 17

6. Target and horn development 22

7. Neutrino beam-lines $\quad 23$

7.1. NuMI 23

7.2. Beam towards DUSEL 24

8. Event rate calculations $\quad 25$

8.1. NuMI off-axis locations 25

8.2. Wide band beam towards DUSEL 26

9. Detector Requirements 29

9.1. Off-axis 30

9.2. Detectors at DUSEL $\quad 32$

10. Status of detector simulations

10.1. Water Cherenkov Detector 33

10.2. Liquid Argon Time Projection Chamber 40

11. Status of detector design and technology 41

11.1. Water Cherenkov conceptual Design 41

11.2. Liquid Argon TPC Conceptual Design 43

12. Overburden and shielding 45

13. Analysis of sensitivity to oscillation parameters 47

13.1. Sensitivity of a FNAL to DUSEL based program 48

13.1.1. Water Cherenkov Detector $\quad 49$

13.1.2. Liquid Argon Detector 
13.2. Sensitivity of a NuMI based off axis program $\quad 61$

13.3. Comparison of sensitivity estimates $\quad 63$

14. Sensitivity to non-accelerator physics $\quad 72$

14.1. Improved Search for Nucleon Decay 72

14.2. Observation of Natural Sources of Neutrinos $\quad 74$

14.3. Depth requirements for non-accelerator physics 77

$\begin{array}{ll}\text { 15. Results and Conclusions } & 78\end{array}$

15.1. Brief comparison of experimental approaches 80

15.2. Project timescales $\quad 81$

16. Acknowledgments $\quad 84$

A. Answers to questions raised by NUSAG

B. NuSAG Charge $\quad 93$

C. Charge to this working group 96

$\begin{array}{lr}\text { D. Study group membership } & 98\end{array}$

E. Relevant resources and URLs for the study group 99

F. Schedule of meetings and report preparation 100

$\begin{array}{lr}\text { References } & 101\end{array}$ 


\section{EXECUTIVE SUMMARY}

This report provides the results of an extensive and important study of the potential for a U.S. scientific program that will extend our knowledge of neutrino oscillations well beyond what can be anticipated from ongoing and planned experiments worldwide. The program examined here has the potential to provide the U.S. particle physics community with world leading experimental capability in this intensely interesting and active field of fundamental research. Furthermore, this capability is not likely to be challenged anywhere else in the world for at least two decades into the future. The present study was initially commissioned in April 2006 by top research officers of Brookhaven National Laboratory and Fermilab and, as the study evolved, it also provides responses to questions formulated and addressed to the study group by the Neutrino Scientific Advisory Committee (NuSAG) of the U.S. DOE and NSF. The participants in the study, its Charge and history, plus the study results and conclusions are provided in this report and its appendices. A summary of the conclusions is provided in this Executive Summary.

The study of neutrino oscillations has grown continuously as its key impact on particle physics and various aspects of cosmology have become increasingly clear. The importance of this fundamental physics was recognized by the National Research Council[1] and the Office of Science and Technology Policy[2], and its national budget priority has been established in a joint OSTPOMB policy memorandum in 2005[3]. In fact, as the present study confirms, it is now possible to design practical experiments that are capable of measuring all the parameters that characterize 3-generation neutrino oscillations, including the demonstration of CP-violation for a significant range of parameter values beyond present limits. Also, one of the experimental approaches, in which the detector (regardless of technology) is deployed deep underground, considered in this study has the potential to contribute, to a significant improvement of our knowledge about nucleon decay and natural sources of neutrinos.

The two experimental approaches studied here are complex in their detailed technical realization, comprising several detector technologies, various specific neutrino beam designs and different measurement strategies. They have in common, however, the exploitation of experimental baselines of $\sim 1000 \mathrm{~km}$ (a key advantage of a U.S. based program) and both approaches make effective use of existing Fermilab accelerator infrastructure with modest upgrades. The experimental detectors required are very massive (in the several hundred kiloton range) because the interaction rates are small. The designs for such detectors vary from already-demonstrated at a scale of 50 kTon (Super Kamiokande) to somewhat speculative (large liquid Argon). In both cases, significant $R \& D$ is still needed to demonstrate feasibility and obtain a reliable cost estimate for the scale needed here. The study has shown however, that it will be feasible and practical to carry out the desired program of important neutrino physics, perhaps together with improved nucleon decay and 
natural neutrino investigations in the same neutrino detector.

The output of the present study is twofold: 1) technical results and conclusions that report the results of the study and address the charge letter; 2) answers to the 15 questions posed to the study group by the NuSAG Committee. These two outputs comprise more than 50 pages of detailed commentary and they are provided in full in the body of the report and Appendix A. Here, we attempt to provide a somewhat condensed version of the study results and conclusions while urging the reader to consult the full text of the report on any points that may appear to be questionable or unclear. The summary results and conclusions were discussed and agreed to at the September 17, 2006 meeting of the study group.

Results and Conclusions:

- Very massive detectors with efficient fiducial mass of $>100$ kTon are needed for the accelerator long baseline neutrino program of the future. We define efficient fiducial mass as fiducial mass multiplied by the signal efficiency. For accelerator based neutrino physics, this could correspond to several hundred kTon if the detector is a water Cherenkov detector and $>100 \mathrm{kTon}$ if it is liquid argon TPC with high expected efficiency. These detectors could be key shared research facilities for the future particle, nuclear and astrophysics research programs. Such a detector(s) could be used with a long baseline neutrino beam from an accelerator laboratory to determine (or bound) leptonic $\mathrm{CP}$ violation and measure all parameters of neutrino oscillations. At the same time, if located in a low background underground environment, it would have additional physics capabilities for proton decay and continuous observation of natural sources of neutrinos such as supernova or other astrophysical sources of neutrinos.

- The Phase-II program will need considerable upgrade to the current accelerator intensity from FNAL. Main Injector accelerator intensity upgrade to $\sim 700 \mathrm{~kW}$ is already planned for Phase-I of the program (NOvA). A further upgrade to $1.2 \mathrm{MW}$ is under design and discussion as described briefly in this report. The phase-II program could be carried out with these planned upgrades. Any further improvements, perhaps with a new intense source of protons, will obviously increase the statistical sensitivity and measurement precision.

- A water Cherenkov detector of multi-100kTon size is needed to obtain sufficient statistical power to reach good sensitivity to $\mathrm{CP}$ violation. This requirement is independent of whether one uses the off-axis technique or the broadband technique in which the detector is housed in one of the DUSEL sites.

- High signal efficiency at high energies and excellent background reduction in a liquid argon TPC allows the size of such a detector to be smaller by a factor of 3 compared to a water 
Cherenkov detector for equal sensitivity. Such a detector is still quite large.

- The water Cherenkov technology is well established. The issues of signal extraction and background reduction were discussed and documented at length in this study. The needed background reduction is achievable and well understood for the broadband beam discussed in this report, but not yet fully optimized. Key issues for scaling up the current generation of water Cherenkov detectors (Super-Kamiokande, SNO, etc.) and locating such detectors in underground locations in DUSEL are well understood. The cost and schedule for such a detector could be created with high degree of confidence. A first approximation for this was reported to the workshop.

- For a very large liquid argon time projection detector key technical issues have been identified for the building of the detector. A possible development path includes understanding argon purity in large industrial tanks, mechanical and electronics issues associated with long wires, and construction of at least one prototype in the mass range of $1 \mathrm{kTon}$.

- In the course of this study, we have examined the surface operation of the proposed massive detectors for accelerator neutrino physics. Water Cherenkov detectors are suitable for deep underground locations only. Surface or near-surface operation of liquid argon TPCs is possible but requires that adequate rejection of cosmic rays be demonstrated. Surface or near-surface operation capability is essential for the off-axis program based on the existing NuMI beam-line because of the geographic area through which the beam travels.

- Additional detailed technical conclusions of the study are noted in the Results and Conclusions section of this report. These results could influence the detailed design of the specific program selected. 


\section{INTRODUCTION}

This report details the activities and the results of a several month long study on long baseline neutrinos. This workshop (named the US joint study on long baseline neutrinos) was sponsored by both Fermi National Accelerator Laboratory and Brookhaven National Laboratory.

Charge: This study grew out of two parallel efforts. An earlier attempt to create a joint FNAL/BNL task force on long baseline neutrinos was initiated by the management of these two laboratories. Later the need arose to provide input to the neutrino scientific advisory committee (NuSAG) which was asked to address the APS study's recommendation for a next generation neutrino beam and detector configuration. The NuSAG charge is in Appendix B. The APS study report can be obtained from http://www.aps.org/neutrino/. The study principals created a charge with specific scenarios for an accelerator based program. The charge from the chairs of the study is in Appendix C.

Membership: Although the study group was asked to mainly focus on a next generation program within the US, participation from the world wide community of particle physicists was sought. In particular, physicists engaged in a European equivalent of this study (the International Scoping Study: http://www.hep.ph.ic.ac.uk/iss/) were kept abreast of our progress. The list of physicists who participated in this study by either contributing written material, presentations, or discussion is in at http://nwg.phy.bnl.gov/fnal-bnl/.

The membership was divided into several subgroups. The accelerator subgroup studied and summarized the proton intensities available mainly from FNAL. The neutrino beam subgroup summarized the neutrino beam intensities and event rates for various possibilities. The water Cherenkov subgroup summarized the current understanding of the conceptual design of such a detector as well as the state of the art in simulating and reconstructing events in such a detector. The liquid argon detector subgroup studied the capabilities of such a detector as well as the feasibility of building a detector large enough to collect sufficient numbers of events. The results from each of these groups is either in presentations, technical documents prepared in the near past, or in technical documents prepared specifically for this study.

Scope of the work: As specified in Appendix C, the scope of our work was limited to conventional horn focused accelerator neutrino beams from US accelerator laboratories. It was asked that we study a next generation program by placing massive detectors either off-axis on the surface for the NuMI beam-line at FNAL, or by building a new intense beam-line aimed towards a new deep underground science laboratory (DUSEL) in the western US. The detector technology to be considered was either a water Cherenkov detector or a liquid Argon time projection chamber. The international scoping study (ISS) on the other hand focused on new technology ideas such as beta-beams and muon storage ring based neutrino factories. 
- In the following we will refer to the NOvA program using the NuMI off axis beam as Phase-I. We will not study or comment on this phase extensively since it has been previously reviewed extensively, but it will be necessary for us to use the extensive existing material for this phase to study the next two items.

- An upgraded off-axis program with multiple detectors, including a massive liquid argon detector, as Phase-II(option A). There could be various versions of Phase-II(option A), with or without a liquid argon detector, with a water Cherenkov detector, and/or detectors at various locations off axis. We will attempt to elaborate on all of these.

- A program using a new beam-line towards DUSEL, housing a massive multipurpose detector, either a water Cherenkov or a liquid argon detector, will be called Phase-II(option B). We will provide information on the DUSEL candidate sites as well as the two options for a multipurpose detector.

Schedule: The study followed the schedule outlined in section F. The first meeting of the FNAL and BNL management that led to the study was held at BNL on November 14, 2005. The charge of the workshop which defined the scope of the work was finalized after the meeting on March 6-7, 2006. It was decided at this meeting that since the time for the report was short, it was best to create small subgroups to work on individual papers for the study. These papers would be distributed to the study group as well as the NuSAG committee as they were prepared.

A set of presentations were made to the NuSAG committee on May 20, 2006. Results from ongoing work was reviewed at this meeting. We selected July 15, 2006 as a deadline for preparation of the individual papers. Many, but not all, papers were prepared by July 15, and were distributed by web-site (http://nwg.phy.bnl.gov/fnal-bnl).

After discussion within the working group a summary report (this report) was commissioned. The contents of this report were reviewed by the study group on September 16-17, 2006. The deadline for delivering this report to NuSAG is October, 2006.

\section{PHYSICS GOALS OF A PHASE-II PROGRAM}

There is now an abundance of evidence that neutrinos oscillate among the three known flavors $v_{e}, v_{\mu}$ and $v_{\tau}$, thus indicating that they have masses and mix with one another. Indeed, modulo an anomaly in the LSND experiment, all observed neutrino oscillation phenomena are well described by the 3 generation mixing 


$$
\begin{aligned}
& \left(\begin{array}{l}
\mid v_{e}> \\
\mid v_{\mu}> \\
\mid v_{\tau}>
\end{array}\right)=\mathbf{U}\left(\begin{array}{l}
\mid v_{1}> \\
\mid v_{\mathbf{2}}> \\
\mid v_{\mathbf{3}}>
\end{array}\right) \\
& U=\left(\begin{array}{ccc}
\mathbf{c}_{12} \mathbf{c}_{13} & \mathbf{s}_{12} \mathbf{c}_{13} & \mathbf{s}_{13} \mathbf{e}^{-\mathbf{i} \delta} \\
-\mathbf{s}_{12} \mathbf{c}_{23}-\mathbf{c}_{12} \mathbf{s}_{23} \mathbf{s}_{13} \mathbf{e}^{\mathrm{i} \delta} & \mathbf{c}_{12} \mathbf{c}_{23}-\mathbf{s}_{12} \mathbf{s}_{23} \mathbf{s}_{13} \mathbf{e}^{\mathbf{i} \delta} & \mathbf{s}_{23} \mathbf{c}_{13} \\
\mathbf{s}_{12} \mathbf{s}_{23}-\mathbf{c}_{12} \mathbf{c}_{23} \mathbf{s}_{13} \mathbf{e}^{\mathrm{i} \delta} & -\mathbf{c}_{12} \mathbf{s}_{23}-\mathbf{s}_{12} \mathbf{c}_{23} \mathbf{s}_{13} \mathbf{e}^{\mathrm{i} \delta} & \mathbf{c}_{23} \mathbf{c}_{13}
\end{array}\right) \\
& c_{i j}=\cos \theta_{i j} \quad, \quad s_{i j}=\sin \theta_{i j}, \quad i, j=1,2,3
\end{aligned}
$$

with $\mid v_{i}>, i=1,2,3$, the neutrino mass eigenstates.

Atmospheric neutrino oscillations are governed by a mass squared difference $\Delta m_{32}^{2}=m_{3}^{2}-m_{2}^{2}=$ $\pm 2.5 \times 10^{-3} \mathrm{eV}^{2}$ and mixing angle $\theta_{23} \simeq 45^{\circ}$; findings that have been confirmed by accelerator generated neutrino beam studies at Super-Kamiokande and MINOS.

As yet, the sign of $\Delta m_{32}^{2}$ is undetermined. The so-called normal mass hierarchy, $m_{3}>m_{2}$, suggests a positive sign which is also preferred by theoretical models. However, a negative value (or inverted hierarchy) can certainly be accommodated and if that is the case, the predicted rates for neutrino-less double beta decay will likely be larger and more easily accessible experimentally. Resolving the sign of the mass hierarchy is an extremely important issue. In addition, the fact that $\theta_{23}$ is large and near maximal is also significant for model building. Measuring that parameter with precision is highly desirable.

In the case of solar and reactor neutrino oscillations, one finds $\Delta m_{21}^{2}=m_{2}^{2}-m_{1}^{2} \simeq 8 \times 10^{-5} \mathrm{eV}^{2}$ and $\theta_{12} \simeq 32^{\circ}$. Again, the mixing angle is relatively large (relative to the analogous Cabbibo angle $\simeq 13^{\circ}$ of the quark sector). In addition, $\Delta m_{21}^{2}$ is large enough, compared, to $\Delta m_{32}^{2}$, to make long baseline neutrino oscillation searches for $\mathrm{CP}$ violation feasible and could yield positive results, i.e. the stage is set for a future major discovery ( $\mathrm{CP}$ violation in the lepton sector).

Currently, we know nothing about the value of the CP violating phase $\delta \quad\left(0<\delta<360^{\circ}\right)$ and only have an upper bound on the as yet unknown mixing angle $\theta_{13}\left(\theta_{13}<13^{\circ}\right)$

$$
\sin ^{2} 2 \theta_{13} \leq 0.2
$$

The value of $\theta_{13}$ is likely to be determined by the coming generation of reactor $\bar{v}_{e}$ disappearance and accelerator based $v_{\mu} \rightarrow v_{e}$ appearance experiments if $\sin ^{2} 2 \theta_{13} \geq 0.01$. Knowledge of $\theta_{13}$ and $\delta$ would complete our determination of the 3 generation lepton mixing matrix and provide a measure of leptonic CP violation via the Jarlskog invariant. 


$$
J_{C P} \equiv \frac{1}{8} \sin 2 \theta_{12} \sin 2 \theta_{13} \sin 2 \theta_{23} \cos \theta_{13} \sin \delta .
$$

If we use the above limit for $\theta_{13}$ then $J_{C P}^{\text {Leptonic }}<0.05 \times \sin \delta$, which could easily turn out to be much larger than the analogous quark degree of CP violation $J_{C P}^{\text {Quarks }} \simeq 3 \times 10^{-5}$.

Based on our current knowledge and future goals, a phase II neutrino program should include:

- Completing the measurement of the leptonic mixing matrix,

- Study of CP violation,

- Determining the values of all parameters with high precision including $J_{C P}$ as well as the sign of $\Delta m_{32}^{2}$

- Searching for exotic effects perhaps due to sterile neutrino mixing, extra dimensions, dark energy etc.

Of the above future neutrino physics goals, the search for and study of $\mathrm{CP}$ violation is of primary importance and should be our main objective for several reasons which we briefly outline.

$\mathrm{CP}$ violation has so far only been observed in the quark sector of the Standard Model. Its discovery in the leptonic sector should shed additional light on the role of $\mathrm{CP}$ violation in Nature. Is it merely an arbitrary consequence of inevitable phases in mixing matrices or something deeper? Perhaps, most important, unveiling leptonic CP violation is particularly compelling because of its potential connection with the observed matter-antimatter asymmetry of our Universe, a fundamental problem at the heart of our existence. The leading explanation is currently a leptogenesis scenario in which decays of very heavy right-hand neutrinos created in the early universe give rise to a lepton number asymmetry which later becomes a baryon-antibaryon asymmetry via the B-L conserving 't Hooft mechanism of the Standard Model at weak scale temperatures.

Leptogenesis offers an elegant, natural explanation for the matter-antimatter asymmetry; but it requires some experimental confirmation of its various components before it can be accepted. Those include the existence of very heavy right-handed neutrinos as well as lepton number and $\mathrm{CP}$ violation in their decays.

Direct detection of those phenomena is highly unlikely; however, indirect connections may be established by studying lepton number violation in neutrinoless double beta decay and CP violation in ordinary neutrino oscillations. Indeed, such discoveries will go far in establishing leptogenesis as a credible, even likely scenario. For that reason, neutrinoless double beta decay and leptonic $\mathrm{CP}$ violation in neutrino oscillations are given very high priorities by the particle and nuclear physics communities. 
Designing for $\mathrm{CP}$ violation studies in next generation neutrino programs has other important benefits. First, the degree of difficulty needed to establish CP violation and determine $J_{C P}^{\text {leptonic }}$ is very demanding but doable. It requires an intense proton beam of about 1-2 MW and a very large detector $(250 \sim 500$ kton Water Cherenkov or a liquid argon detector of size $\sim 100$ kTon which could be equivalent in sensitivity due to its better performance). Such an ambitious infrastructure will allow very precise measurements of all neutrino oscillation parameters as well as the sign of $\Delta m_{32}^{2}$ via $v_{\mu} \rightarrow v_{\mu}$ disappearance and $v_{\mu} \rightarrow v_{e}$ appearance studies. It will also provide a sensitive probe of "New Physics" deviations from 3 generation oscillations, perhaps due to sterile neutrinos, extra dimensions, dark energy or other exotic effects.

A well instrumented very large detector, in addition to its accelerator based neutrino program, could be sensitive to proton decay which is one of the top priorities in fundamental science. Assuming that it is located underground and shielded from cosmic rays, it can push the limits on proton decay into modes such as $p \rightarrow e^{+} \pi^{0}$ to $10^{35} \mathrm{yr}$ sensitivity or beyond, a level suggested by gauge boson mediated proton decay in super-symmetric GUTS. Indeed, there is such a natural marriage between the requirements to discover leptonic CP violation and / or see proton decay (i.e. an approximately 500 kTon water Cherenkov detector) that it could be hard to imagine undertaking either effort without being able to do the other.

Such a large detector would also have additional physics capabilities. It could study atmospheric neutrino oscillations with very high statistics and look for the predicted relic supernova neutrinos left over from earlier epochs in the history of the Universe, a potential source of cosmological information. Also, if a supernova should occur in our galaxy (expected about every 30 years), such a detector would see about 100,000 neutrino events. In addition, it could be used to look for signals of $n-{ }^{-} n$ oscillations in nuclei and highly penetrating GUT magnetic monopoles which would leave behind a trail of monopole catalyzed proton decays.

The physics potential of a very large underground detector is extremely rich. The fact that it can also be used to determine (or bound) leptonic $\mathrm{CP}$ violation and measure all facets of neutrino oscillations gives such a facility outstanding discovery potential. It would be an exciting, central component of the world's particle physics program for many decades. On the other hand, a staged approach using existing beam facilities should also be explored to determine an optimum strategy.

\section{STRATEGIES FOR THE PHASE-II PROGRAM USING A CONVENTIONAL BEAM}

In this section we will describe the essential features of an off-axis narrow band beam versus an on-axis broad band beam. We will then briefly summarize how these features can be used to extract the CP violation effect as well as all the other parameters of importance in neutrino oscillations.

Throughout this report we are concerned with conventional horn focused beams in the US: 
the existing NuMI beam at FNAL or a new super neutrino beam that could be optimized for a detector at a new deep underground national laboratory (DUSEL) with a possible large detector (either underground or on the surface). The measurement of most interest is always the appearance measurement, $v_{\mu} \rightarrow v_{e}$, for which the horn focused beam has a limitation from the irreducible background of $v_{e}$ contamination in the beam. The level of contamination depends on neutrino energy and also the beam design and the off-axis angle, but it is in the range of $\sim 0.5-1 \%$ for most practical beams. This contamination comes from decays of muons and kaons in the beam. These cannot be completely eliminated. The second source of background is neutral current events that mimic electron showers. This background is considered reducible by detector design. In particular, a fine grained detector such as a liquid argon TPC detector will be capable of reducing such background to very small levels. Most of the remaining report will be concerned with the best strategy for obtaining sufficient signal events while reducing these backgrounds. In this section we will not discuss the issues of backgrounds in detail, but give a guide to the signal spectra, event rates and comment on the implications.

Figures 1, 2, 3, and 4 show the spectra of concern. Care is required in comparing these plots because they are plotted on a logarithmic energy scale. The normalization is per GeV of neutrino energy per kTon of detector mass per $M W \times 10^{7} \mathrm{sec}$ protons of the appropriate energy on target.

These spectra were obtained by detailed simulations using the GNuMI computer program[4]. For these figures a simple recipe was used to obtain charged current event rate [5]: a cross section of $0.8 \times 10^{-38} \mathrm{~cm}^{2} / \mathrm{GeV}\left(0.35 \times 10^{-38} \mathrm{~cm}^{2} / \mathrm{GeV}\right.$ for anti-neutrinos $)$ was used above $0.5 \mathrm{GeV}$ and the quasi-elastic cross section was used below $0.5 \mathrm{GeV}$. There could be small differences due to the detector target type (water, argon, etc.), but this is a good approximation [6]. For figures 1 and 2 we have used the low energy (LE) setting of the NuMI beam configuration which gives a better flux at the $40 \mathrm{~km}$ site. Reference [5] contains spectra for other choices. For all the off-axis spectra $120 \mathrm{GeV}$ protons were used and the normalization is for $M W \times 10^{7} \mathrm{sec}$ protons; for 120 $\mathrm{GeV}$ protons this corresponds to $5.2 \times 10^{20}$ protons.

For Figures 3 and 4, the GNuMI program was modified for a wide band low energy (WBLE) design for the horns as well as a new decay tunnel with $4 \mathrm{~m}$ diameter and $400 \mathrm{~m}$ length; these are described in detail in [7]. For the WBLE beam, there is a choice of running with protons from $40 \mathrm{GeV}$ to $120 \mathrm{GeV}$. For these plots we have chosen $60 \mathrm{GeV}$ protons. The normalization is for $M W \times 10^{7} \mathrm{sec}$ protons of $60 \mathrm{GeV}$. The spectra shown here should not be considered optimum. After thorough design and optimization there could be modest improvements, but at this point we are confident that these numbers are sufficiently good for this review.

For Figures 1 to 4 we have superimposed the expected probability of $v_{\mu} \rightarrow v_{e}$ conversion for the appropriate distance and for the following oscillation parameters: $\Delta m_{32}^{2}=0.0025 \mathrm{eV}^{2}, \Delta m_{21}^{2}=8 \times$ $10^{-5} \mathrm{eV}^{2}, \sin ^{2} 2 \theta_{12}=0.86, \sin ^{2} 2 \theta_{23}=1.0$, and $\sin ^{2} 2 \theta_{13}=0.04$; the curves are for several choices 
of the CP phase and the left and right hand side plots are for the two different mass orderings. These parameters will be used for many plots and tables in this study.

In Table I we have calculated the rate of electron appearance events for various scenarios by integrating the spectrum together with the appearance probability. This event rate is for all charged current events; no detector efficiency factors are applied. A detector with efficient fiducial mass of 100 kTon is assumed with $\sim 10^{7} \mathrm{sec}$ of running time with $1 \mathrm{MW}$ of proton beam. No consideration for backgrounds, energy thresholds, or resolution effects are in this table.

Also note that Figures 1 to 4 do not show the event rates from anti-neutrinos. These can be obtained from the study web-site [8]. We include anti-neutrino rates and spectra in later sections with more detail. We have included anti-neutrino event rate in Table I.

After considering the figures and the table we make the following observations:

- For simplicity we look at the electron neutrino event rate at $\delta_{C P}=0$ and compare it to $\delta_{C P}=90^{\circ}$. In the limit that one has resolved the mass hierarchy using the anti-neutrino data, the modulation of the neutrino rate with $\delta_{C P}$ will give us the CP parameter measurement that we seek. One can immediately see that the size of the CP effect for the maximum $\mathrm{CP}\left(90^{\circ}\right)$ is approximately $3 \sigma$. To achieve this within a year of running (with no consideration for efficiencies, backgrounds, etc.) the efficient fiducial mass of the detector must be 100kTon range if the accelerator power is limited to be $\sim 1 \mathrm{MW}$. This conclusion is regardless of the eventual choice for the beam-line.

- The size of the $\mathrm{CP}$ effect (for the maximum $90^{\circ}$ ) increases modestly from $\sim 3 \sigma$ for the off axis $(810 \mathrm{~km}) 12 \mathrm{~km}$ option to about $4.8 \sigma$ for $2500 \mathrm{~km}$. Much of this increase can be traced to the large CP effect at the higher oscillation nodes that become available for the larger distances. The loss of statistics due to distance (as $1 / L^{2}$ ) is largely compensated by the increase in the strength of the CP related signal $[9,10]$. By combining the $40 \mathrm{~km}$ off-axis rates with the $12 \mathrm{~km}$ there is also a modest improvement in the overall $\mathrm{CP}$ measurement. Nevertheless, for the choice of spectra in this report, the baseline length related effects for a $\mathrm{CP}$ measurement are not dramatic for the range of choices in this study.

- Remarkably, it should also be noticed that the size of the CP effect in the number of sigma is approximately the same for the different values of $\sin ^{2} 2 \theta_{13}$. It has been pointed out, therefore, that for $\sin ^{2} 2 \theta_{13} \geq 0.003$, which is the range accessible for conventional accelerator beams, the size of the exposure (efficient fiducial mass multiplied by the total incident beam power) needed to obtain a good measurement of the CP parameter is independent of $\theta_{13}$ $[9,11,12]$. This is explained by the following argument. The asymmetry defined by

$$
A \equiv \frac{P\left(v_{\mu} \rightarrow v_{e}\right)-P\left(\bar{v}_{\mu} \rightarrow \bar{v}_{e}\right)}{P\left(v_{\mu} \rightarrow v_{e}\right)+P\left(\bar{v}_{\mu} \rightarrow \bar{v}_{e}\right)}
$$


is proportional to $J_{C P}^{\text {leptonic }}$ and therefore grows linearly with $\sin \theta_{13}$, but $P\left(v_{\mu} \rightarrow v_{e}\right)$ is to leading order proportional to $\sin ^{2} 2 \theta_{13}$ and therefore the statistical figure of merit, the error on the asymmetry $A$ should have little dependence on $\theta_{13}$.

- The size of the matter effect (the difference between the event rate for the two choices of mass ordering) is approximately $3 \sigma$ for the $12 \mathrm{~km}$ off axis location for $\sin ^{2} 2 \theta_{13}=0.02$ for neutrino rates alone. It is a much larger effect for longer baselines. The probability curves show that the effect is large for the first oscillation node in all cases. This effect will clearly compete with the $\mathrm{CP}$ effect and must be determined along with the $\mathrm{CP}$ effect for clarity. The matter effect clearly is much stronger for larger value of $\theta_{13}$, and therefore for a larger value of $\theta_{13}$, it will be easier to determine the mass hierarchy.

- Examination of the probability curves in figure 1 shows that the $12 \mathrm{~km}$ off axis spectrum is sensitive mainly to the first oscillation node. The probability is affected not only by the CP phase, but also by the value of $\theta_{13}$, the mass ordering, the uncertain values of other parameters such as $\Delta m_{32}^{2}$ and $\theta_{23}$. Also note that the probability curves at any particular energy have degeneracies in the $\mathrm{CP}$ phase. These degeneracies have been discussed in the literature [13-15]. Therefore, to make a clean determination of CP violation, one either needs very good energy resolution (to exploit the small energy dependence within the first node) with good statistics, or one needs to perform another measurement at the high oscillation node by placing another detector further off-axis. This is one of the options to be examined in this report.

- Examination of the probability curves in figures 3 and 4 shows that the energy dependence of the probability can be measured in a single detector by creating a beam spectrum that matches the first few nodes over the $>1000 \mathrm{~km}$ long baseline. Obviously, in such a scenario the neutrino energy must be measured in the detector with sufficient resolution while suppressing backgrounds [10]. This is also an option to be considered in this report. An illustration of how the various degeneracies affect the measurement is shown in figure 5 . The figure illustrates the energy dependence for neutrino running only. It is clear that narrow band running will have additional ambiguities. How these can be broken with additional anti-neutrino running or with high statistics and resolution will be discussed later.

- The neutrino event rate is roughly proportional to the total proton beam power; the exact numbers and deviations from this rule will be discussed below. The total power that can be obtained from FNAL Main Injector after upgrades increases with the output proton energy, and therefore it is important to maintain the highest possible proton energy for either the off-axis or on-axis scenarios. For the off-axis experiment the preferred running is at the 

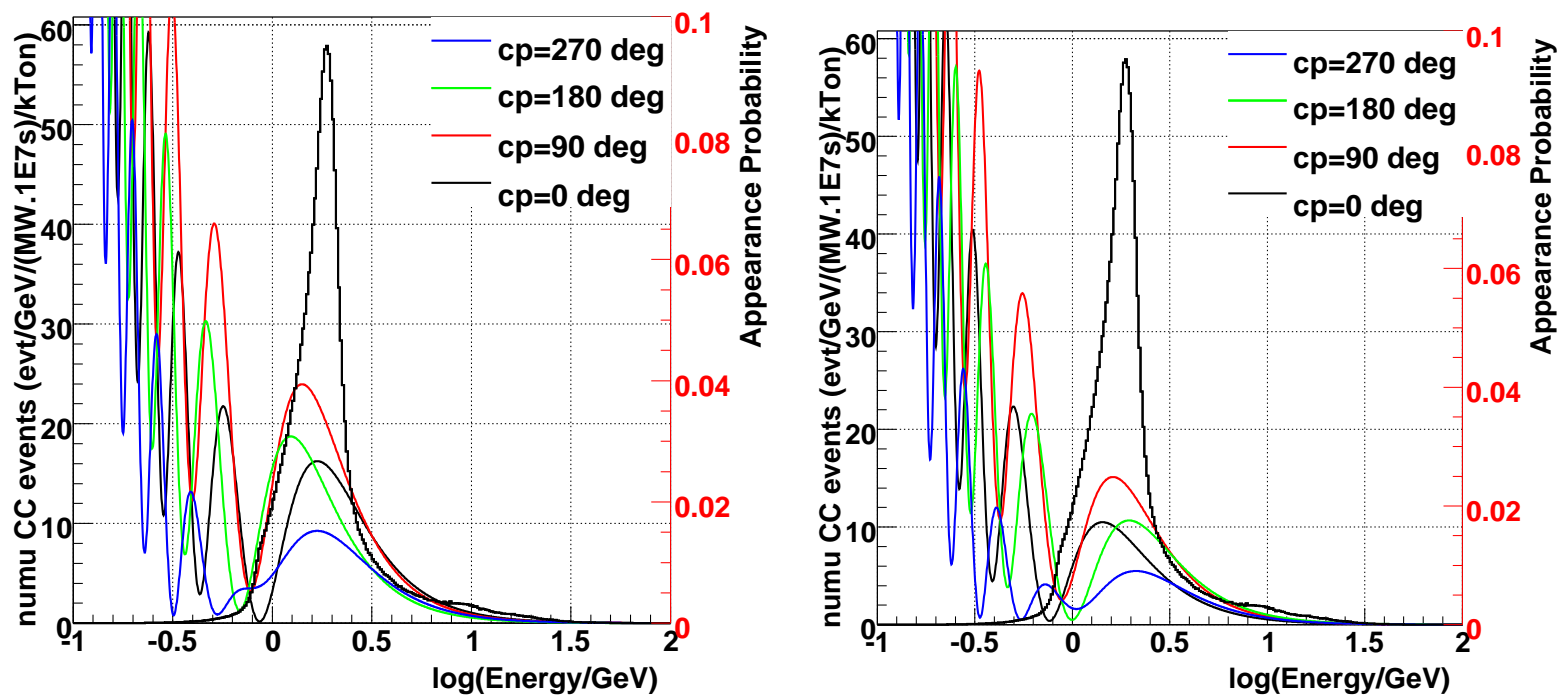

FIG. 1: (in color) Spectrum of charged current $v_{\mu}$ events at a $12 \mathrm{~km}$ off-axis location at $810 \mathrm{~km}$ on the NuMI beam-line. The spectrum is normalized per $\mathrm{GeV}$ per $M W \times 10^{7} \mathrm{sec}$ protons of $120 \mathrm{GeV}$. The low energy (LE) setting of the NuMI beam-line is used for this plot. Overlayed is the probability of $v_{\mu} \rightarrow v_{e}$ conversion for $\sin ^{2} 2 \theta_{13}=0.04$ with rest of the oscillation parameters as described in the text. The left plot is for regular mass ordering and right hand side is for reversed mass ordering. Figure includes no detector effects such as efficiencies, resolution, or backgrounds.

highest, $120 \mathrm{GeV}$, proton energy. For the FNAL-to-DUSEL option, there could be significant advantage at running with lower proton energy. This will reduce the long high energy tail $>5 \mathrm{GeV}$ of the neutrino spectrum. This tail is outside the interesting oscillation region and may contribute increased background in the form of neutral current events that reconstruct to have lower neutrino energy. The event rates given in table I for WBLE assume running with $1 \mathrm{MW}$ of power at $60 \mathrm{GeV}$. In the following we will comment on how $1 \mathrm{MW}$ power can be obtain while maintaining the a flux with low high energy neutrino tail. The easiest way, of course, is by having a small off-axis angle. The flux that could be obtained with a $0.5^{\circ}$ off-axis angle to DUSEL at $1300 \mathrm{~km}$ is shown in figure 6.

We will now explore the above observations in further detail including the feasibility of beams and detectors, current best knowledge on the performance of detectors, and requirements for other physics related applications of these very large detector facilities. 
LE, numu CC, sin2theta13=0.04, $810 \mathrm{~km} / 40 \mathrm{~km}$

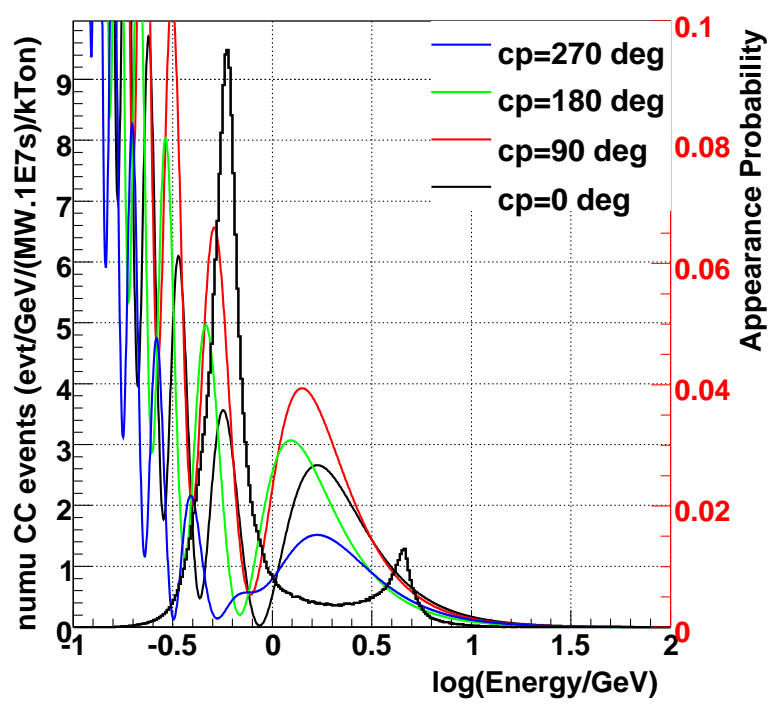

LE, numu CC, sin2theta13=0.04, $810 \mathrm{~km} / 40 \mathrm{~km}$

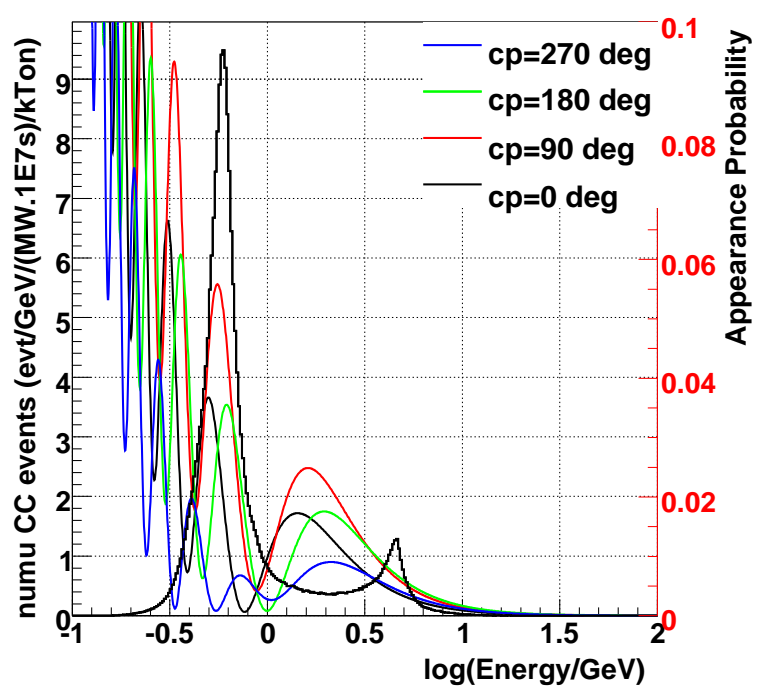

FIG. 2: (color) Spectrum of charged current $v_{\mu}$ events at a $40 \mathrm{~km}$ off-axis location at $810 \mathrm{~km}$ on the NuMI beam-line. The spectrum is normalized per $\mathrm{GeV}$ per $M W \times 10^{7} \mathrm{sec}$ protons of $120 \mathrm{GeV}$. The low energy (LE) setting of the NuMI beam-line is used for this plot. Overlayed is the probability of $v_{\mu} \rightarrow v_{e}$ conversion for $\sin ^{2} 2 \theta_{13}=0.04$ with rest of the oscillation parameters as described in the text. The left plot is for regular mass ordering and right hand side is for reversed mass ordering. Figure includes no detector effects such as efficiencies, resolution, or backgrounds.

\section{ACCELERATOR REQUIREMENTS}

All phases of the envisioned US neutrino accelerator program, Phase-I, Phase-II(option A), or Phase-II(option B), require upgrades to the existing proton accelerator infrastructure in the US. Phase-I upgrades, already planned at FNAL, will increase the Main Injector extracted beam power to $0.7 \mathrm{MW}$ at $120 \mathrm{GeV}$ (this is called "proton plan-2"). The plan to further upgrade the Main Injector to 1.2 MW is called "the SNuMI Project" [16]. Phase-II will benefit from these upgrades.

We have used beam power in the range of $\sim 0.5$ to $2 \mathrm{MW}$ for high energy protons $(>30 \mathrm{GeV})$ in our calculations because this level of beam power is now considered the next frontier for current accelerator technology $[17,22]$ and also necessary to obtain sufficient event rate to perform the next stage of neutrino oscillation physics. The technical limitations arise from the need to control radiation losses, limit the radiation exposure of ground water and other materials, and the feasibility of constructing a target and horn system that can survive the mechanical and radiation damage due to high intensity proton pulses [21].

We quote event rates either in units of $M W \times 10^{7} \mathrm{sec}$ or number of protons on target (POT). A 

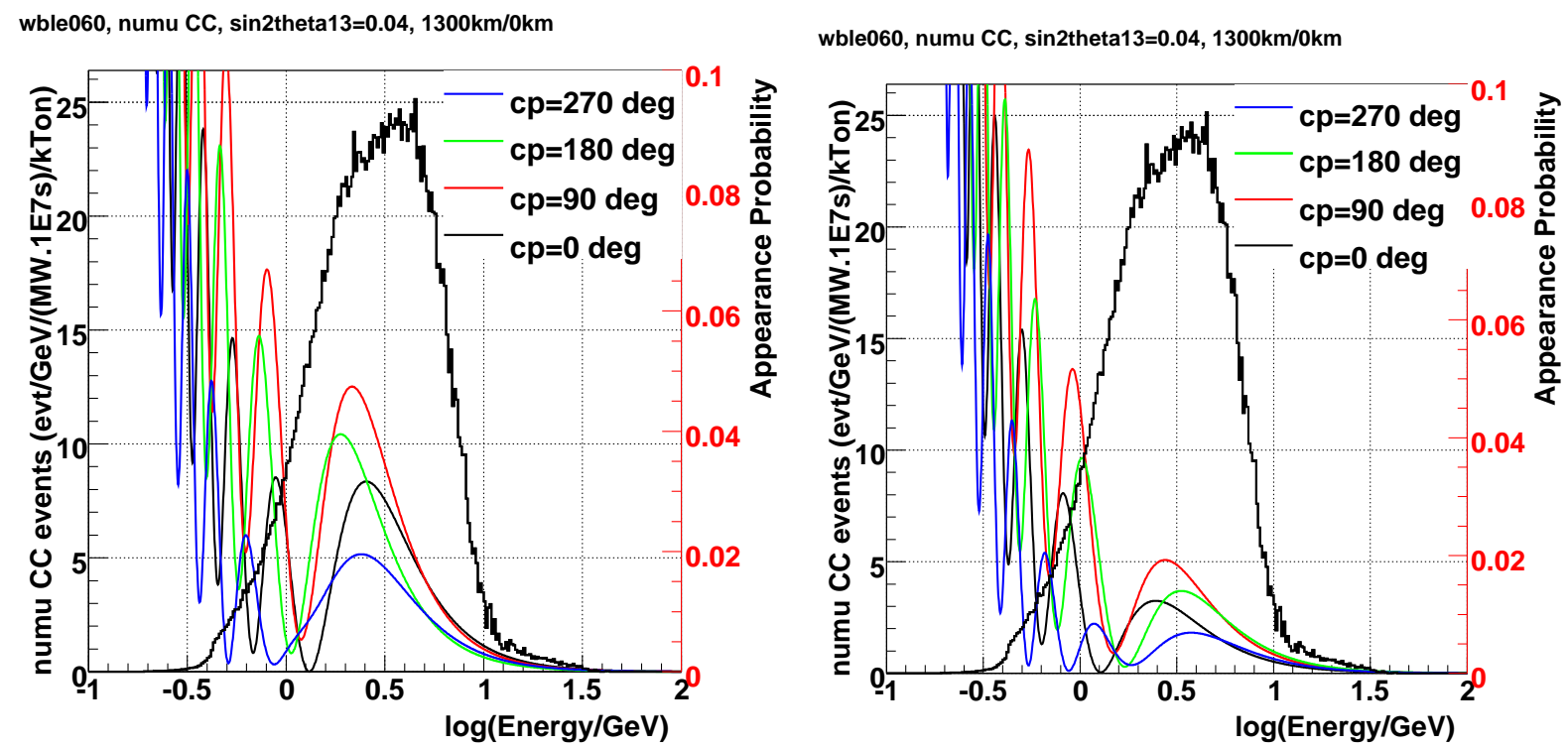

FIG. 3: (color) Spectrum of charged current $v_{\mu}$ events using a new wide band beam from FNAL to a location at $1300 \mathrm{~km}$. The spectrum is normalized per $\mathrm{GeV}$ per $M W \times 10^{7} \mathrm{sec}$ protons of $60 \mathrm{GeV}$. Overlayed is the probability of $v_{\mu} \rightarrow v_{e}$ conversion for $\sin ^{2} 2 \theta_{13}=0.04$ with rest of the oscillation parameters as described in the text. The left plot is for regular mass ordering and right hand side is for reversed mass ordering. Figure includes no detector effects such as efficiencies, resolution, or backgrounds.

convenient formula for conversion is below.

$$
\operatorname{POT}\left(10^{20}\right)=\frac{1000 \times \text { BeamPower }(\mathrm{MW}) \times T\left(10^{7} \mathrm{~s}\right)}{1.602 \times E_{p}(\mathrm{GeV})}
$$

where $T$ is the amount of exposure time in units of $10^{7} s$ and $E_{p}$ is the proton energy. We now briefly summarize the understanding of high energy proton beam power at the two US accelerator laboratories where high intensity proton synchrotrons are operational, Fermilab and Brookhaven.

FNAL Main injector (MI): Discussion is currently underway to increase the total power from the $120 \mathrm{GeV}$ Main Injector (MI) complex after the Tevatron program ends [16, 17]. In this scheme protons from the $8 \mathrm{GeV}$ booster, operating at $15 \mathrm{~Hz}$, will be stored in the recycler (which becomes available after the shutdown of the Tevatron program) while the MI completes its acceleration cycle, which is shortened from the current $2.2 \mathrm{sec}$ to $1.33 \mathrm{sec}$. In a further upgrade the techniques of momentum stacking using the antiproton accumulator, and slip-stacking using the recycler will raise the total intensity in the MI to $\sim 1.2 \mathrm{MW}$ at $120 \mathrm{GeV}$ [18]. In the rest of this report this will be called the SNuMI plan. In the ideal case, the length of the acceleration cycle is proportional to the proton energy, making the average beam power proportional to the final proton energy. However, fixed time intervals in the beginning and the end of the acceleration cycle are required for stable operation. These become important at low energies and reduce the performance below the ideal. 

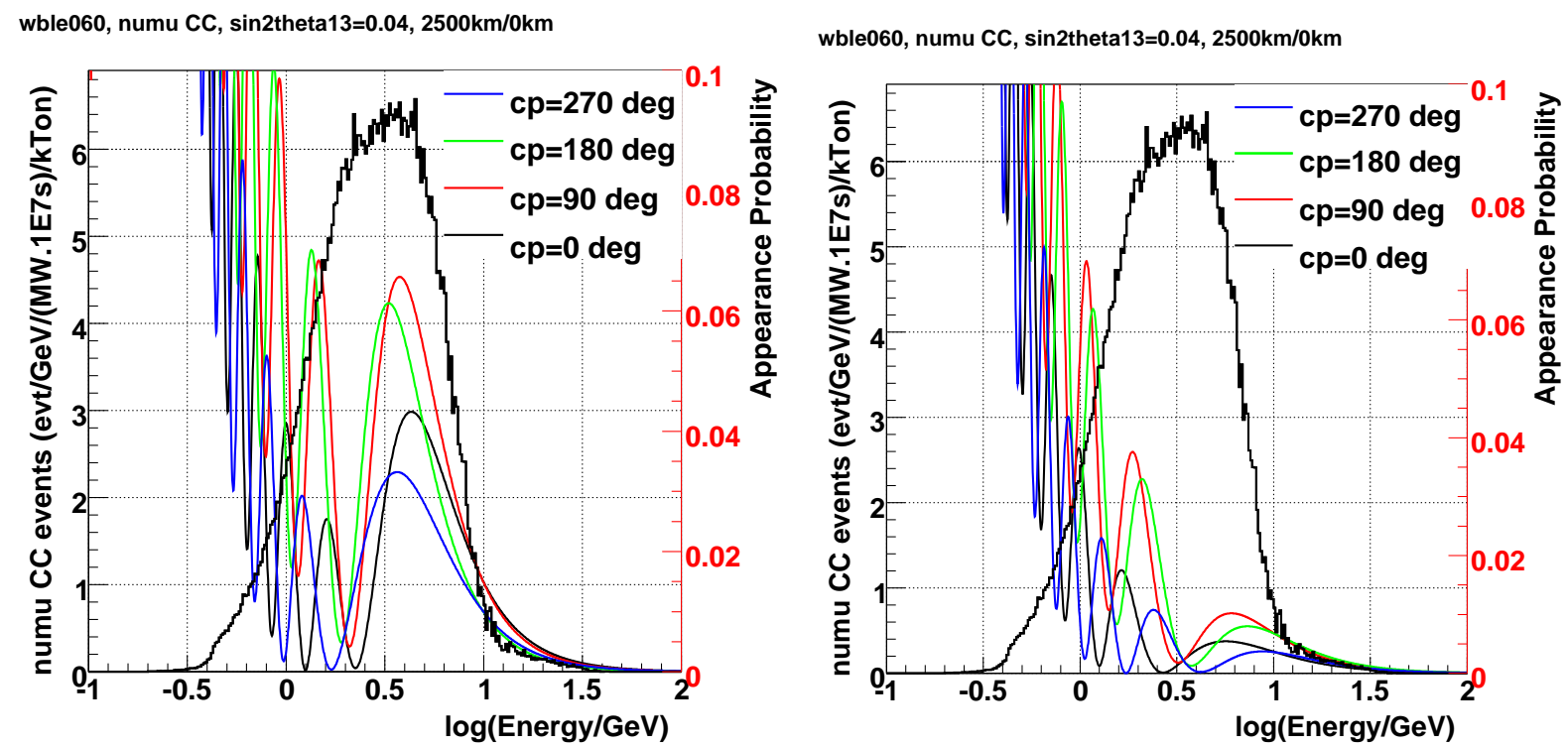

FIG. 4: (color) Spectrum of charged current $v_{\mu}$ events using a new wide band beam from FNAL to location at $2500 \mathrm{~km}$. The spectrum is normalized per $\mathrm{GeV}$ per $M W \times 10^{7} \mathrm{sec}$ protons of $60 \mathrm{GeV}$. Overlayed is the probability of $v_{\mu} \rightarrow v_{e}$ conversion for $\sin ^{2} 2 \theta_{13}=0.04$ with rest of the oscillation parameters as described in the text. The left plot is for regular mass ordering and right hand side is for reversed mass ordering. Figure includes no detector effects such as efficiencies, resolution, or backgrounds.

Current projections suggest that $\sim 0.5 \mathrm{MW}$ operation between $40-60 \mathrm{GeV}$ and $\gtrsim 1 \mathrm{MW}$ operation at $120 \mathrm{GeV}$ is possible.

More ambitious plans at FNAL call for replacing the $8 \mathrm{GeV}$ booster with a new superconducting LINAC that can provide $1.5 \times 10^{14} \mathrm{H}^{-}$ions at $10 \mathrm{~Hz}$ corresponding to $2 \mathrm{MW}$ of total beam power [19]. Some of the $8 \mathrm{GeV}$ ions could be injected into the MI to provide high proton beam power at any energy between 30 and $120 \mathrm{GeV}$; e.g., $40 \mathrm{GeV}$ at $\sim 2 \mathrm{~Hz}$ or $120 \mathrm{GeV}$ at $\sim 0.67 \mathrm{~Hz}$. Such a plan allows for flexibility in the choice of proton energy for neutrino production. This plan will be called the high intensity neutrino source upgrade (HINS).

The projected proton intensity from the main injector for the successive upgrades at FNAL is shown in figure 7[20]. A reviewed base cost estimate (without overhead or contingency factors) for the $700 \mathrm{MW}$ (proton plan-2) upgrade is $\$ 33.4 \mathrm{M}$. The cost of the complete SNuMI plan (to $1.2 \mathrm{MW}$ ) is at the moment only preliminary at $\sim \$ 54 M$. The HINS upgrade is estimated to be approximately $>\$ 300 \mathrm{M}$.

BNL AGS: The BNL Alternating Gradient Synchrotron (AGS) operating at $28 \mathrm{GeV}$ currently can provide about $1 / 6 \mathrm{MW}$ of beam power. This corresponds to an intensity of about $7 \times 10^{13}$ protons in a 2.5 microsecond pulse every 2 seconds. The AGS complex can be upgraded to provide a total proton beam power of $1 \mathrm{MW}$ [22]. The main components of the accelerator upgrade at BNL 

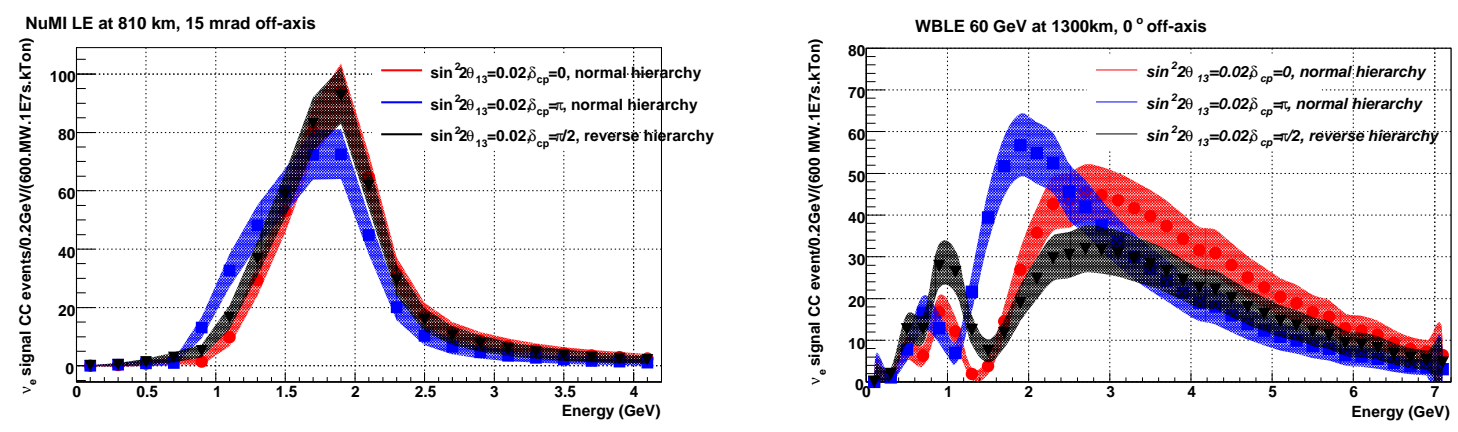

FIG. 5: (in color) Spectrum of charged current $v_{\mu} \rightarrow v_{e}$ events using the LE beam tune at $12 \mathrm{~km}$ off-axis 810 $\mathrm{km}$ location (left) and with a new wide band beam from FNAL (using $60 \mathrm{GeV}$ protons) to a location at 1300 $\mathrm{km}$. The spectra are normalized for $600 \mathrm{MW} \times 10^{7} \mathrm{sec}$ and the width of the band indicates the statistical error. The parameters used for oscillations are shown in the figure, the remaining parameters are as described in the text. Figure includes no detector effects such as efficiencies, resolution, or backgrounds.

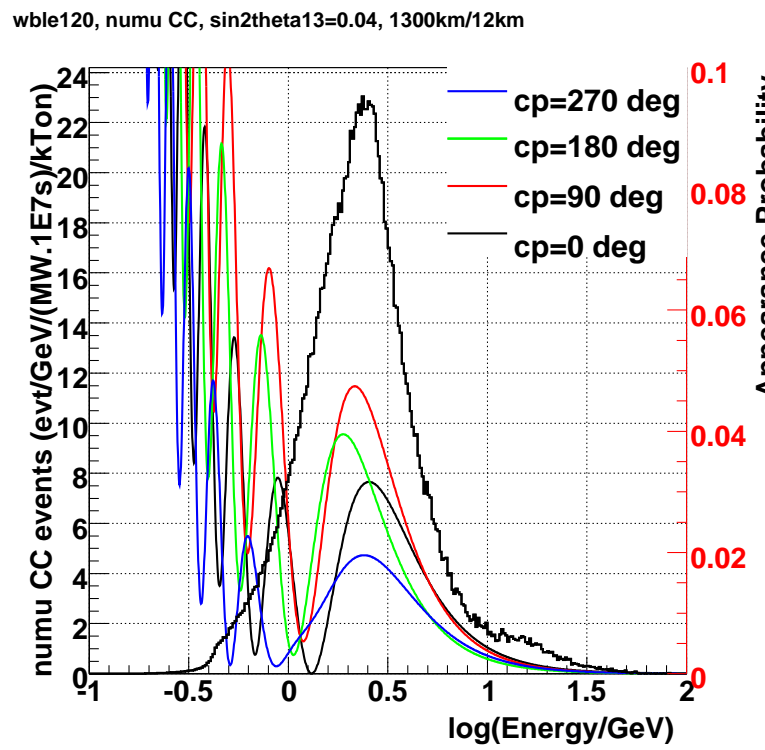

wble120, numu CC, sin2theta13=0.04, 1300km $/ 12 \mathrm{~km}$

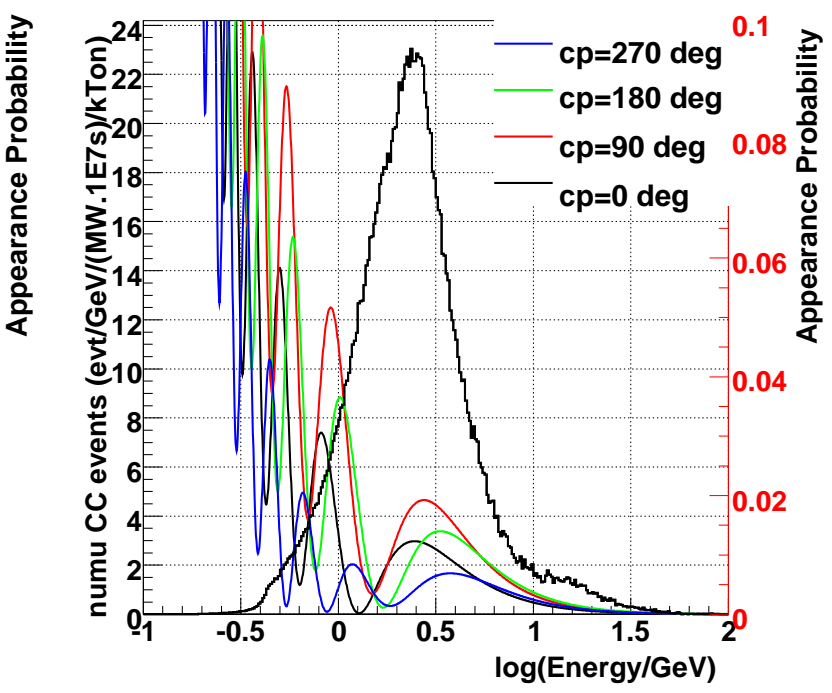

FIG. 6: (in color) Spectrum of charged current $v_{\mu}$ events using a new wide band beam from FNAL to location at $1300 \mathrm{~km}$ with slightly off axis location $(12 \mathrm{~km})$ to reduce the high energy tail. The spectrum is normalized per $\mathrm{GeV}$ per $M W \times 10^{7} \mathrm{sec}$ protons of $120 \mathrm{GeV}$. Overlayed is the probability of $v_{\mu} \rightarrow v_{e}$ conversion for $\sin ^{2} 2 \theta_{13}=0.04$ with rest of the oscillation parameters as described in the text. The left plot is for regular mass ordering and right hand side is for reversed mass ordering. Figure includes no detector effects such as efficiencies, resolution, or backgrounds. 


\begin{tabular}{|c|c|c|c|c|c|c|c|c|c|}
\hline \multirow{3}{*}{ Beam (mass ordering) } & \multirow{3}{*}{$\sin ^{2} 2 \theta_{13}$} & \multicolumn{4}{|c|}{ Neutrino Rates } & \multicolumn{4}{|c|}{ Anti Neutrino Rates } \\
\hline & & \multicolumn{8}{|c|}{$\delta_{C P} \operatorname{deg}$} \\
\hline & & $0^{\circ}$ & $-90^{\circ}$ & $180^{\circ}$ & $+90^{\circ}$ & $0^{\circ}$ & $-90^{\circ}$ & $180^{\circ}$ & $+90^{\circ}$ \\
\hline NuMI LE $12 \mathrm{~km}$ offaxs (+) & 0.02 & 76 & 108 & 69 & 36 & 20 & 7.7 & 17 & 30 \\
\hline NuMI LE 12 km offaxs (-) & 0.02 & 46 & 77 & 52 & 21 & 28 & 14 & 28 & 42 \\
\hline NuMI LE $12 \mathrm{~km}$ offaxs (+) & 0.1 & 336 & 408 & 320 & 248 & 86 & 57 & 78 & 106 \\
\hline NuMI LE 12 km offaxs (-) & 0.1 & 210 & 280 & 224 & 153 & $\mid 125$ & 95 & 126 & 157 \\
\hline NuMI LE $40 \mathrm{~km}$ offaxs (+) & 0.02 & 5.7 & 8.8 & 5.1 & 2.2 & 2.5 & 1.6 & 0.7 & 3.3 \\
\hline NuMI LE 40 km offaxs (-) & 0.02 & 4.2 & 8.0 & 5.7 & 2.0 & 2.3 & 2.2 & 0.8 & 3.6 \\
\hline NuMI LE $40 \mathrm{~km}$ offaxs (+) & 0.1 & 17 & 24 & 15 & 9.4 & 6.7 & 2.8 & 4.6 & 8.5 \\
\hline NuMI LE 40 km offaxs (-) & 0.1 & 12 & 21 & 16 & 7.7 & 6.6 & 3.4 & 6.4 & 9.6 \\
\hline WBLE 1300 km (+) & 0.02 & 141 & 192 & 128 & 77 & 19 & 11 & 18 & 36 \\
\hline WBLE 1300 km (-) & 0.02 & 58 & 111 & 88 & 35 & 45 & 25 & 45 & 64 \\
\hline WBLE $1300 \mathrm{~km}(+)$ & 0.1 & 607 & 720 & 579 & 467 & 106 & 67 & 83 & 122 \\
\hline WBLE 1300 km (-) & 0.1 & 269 & 388 & 335 & 216 & 196 & 154 & 196 & 240 \\
\hline WBLE $2500 \mathrm{~km}(+)$ & 0.02 & 61 & 103 & 88 & 46 & 11 & 4.6 & 4.7 & 11 \\
\hline WBLE 2500 km (-) & 0.02 & 16 & 36 & 33 & 13 & 28 & 15 & 18 & 31 \\
\hline WBLE $2500 \mathrm{~km}(+)$ & 0.1 & 270 & 361 & 328 & 238 & 27 & 13 & 13 & 28 \\
\hline WBLE 2500 km (-) & 0.1 & 47 & 92 & 85 & 39 & 103 & 74 & 80 & 109 \\
\hline
\end{tabular}

TABLE I: This table contains signal event rates after $v_{\mu} \rightarrow v_{e}$ (also for anti-neutrinos) conversion for the various scenarios described. The event rates here have no detector model or backgrounds. The units are charged current events per 100 kTon of detector mass for $1 \mathrm{MW}$ of beam for $10^{7} \mathrm{sec}$ of operation. For NuMI running we assume $120 \mathrm{GeV}$ protons in the LE tune and for WBLE we have assumed $60 \mathrm{GeV}$ protons. The charged current cross sections applied as well as the oscillation parameters used are described in the text.

are a new 1.2 GeV Super-conducting LINAC to provide protons to the existing AGS, and new magnet power supplies to increase the ramp rate of the AGS magnetic field from about $0.5 \mathrm{~Hz}$ to $2.5 \mathrm{~Hz}$. For $1 \mathrm{MW}$ operation $28 \mathrm{GeV}$ protons from the accelerator will be delivered in pulses of $9 \times 10^{13}$ protons at $2.5 \mathrm{~Hz}$. It has been determined that $2 \mathrm{MW}$ operation of the AGS is also possible by further upgrading the synchrotron to $5 \mathrm{~Hz}$ repetition rate and with further modifications to the LINAC and the RF systems. The AGS 1 MW upgrade is estimated to cost \$343M (TEC) including contingency and overhead costs. This cost has been reviewed internally at BNL. 


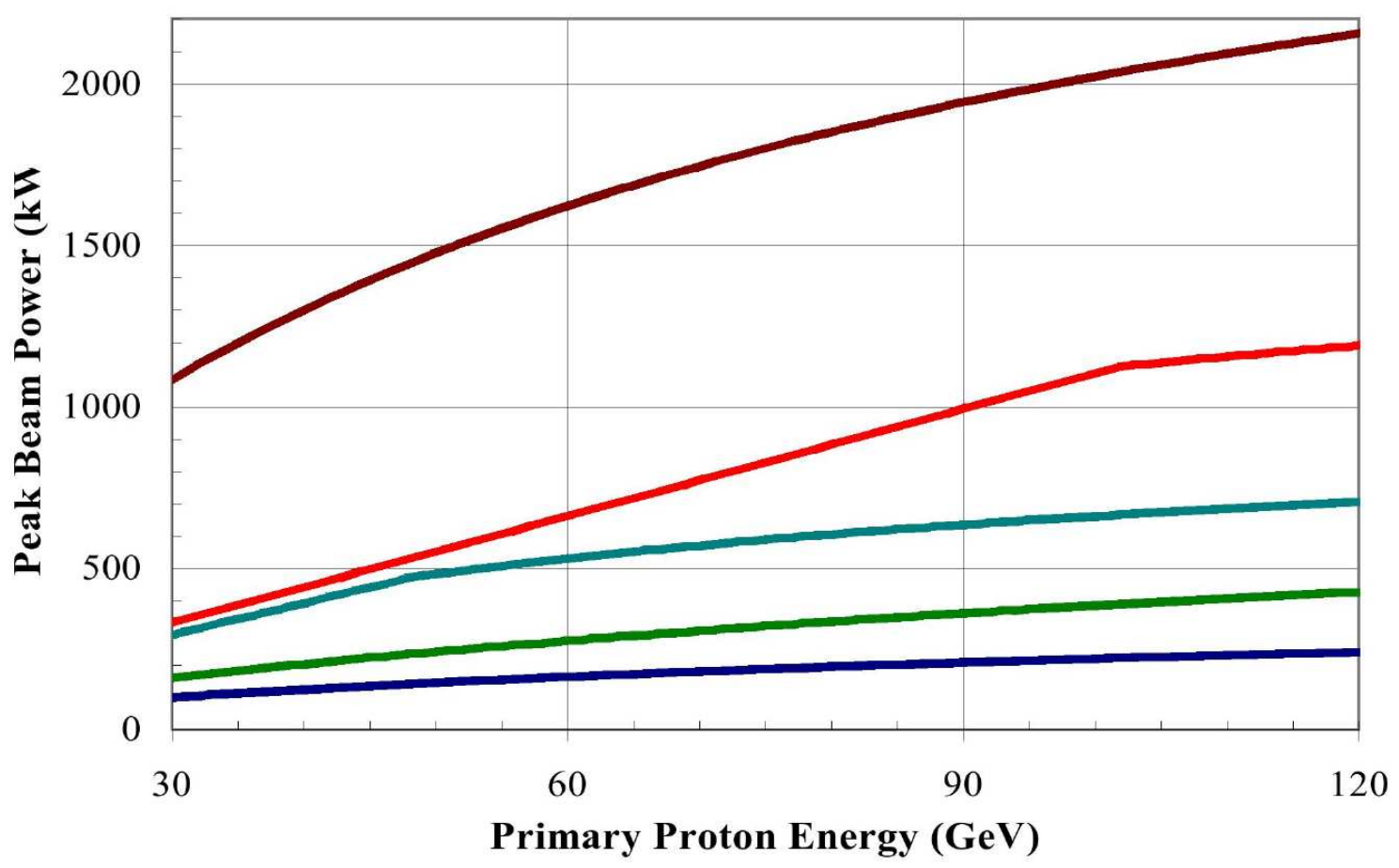

FIG. 7: Proton beam power from the Fermilab main injector as a function proton energy for various scenarios. Lowest (blue) curve is for the current complex running concurrently with the Tevatron. Second (green) curve is for the proton plan upgrades, third (light blue) curve is for SNuMI recycler stage which will take place after the termination of the Tevatron program, fourth (red) is for the accumulator stage upgrades, the last (brown) is for the HINS upgrade which calls for a new $8 \mathrm{GeV}$ LINAC injector.

\section{TARGET AND HORN DEVELOPMENT}

All phases of the envisioned US neutrino accelerator program, Phase-I, Phase-II(option A), or Phase-II(option B), require substantial development for a new target capable of operating at high proton intensities and perhaps new focusing horn optics.

Current understanding of targets, and $R \& D$ in progress is summarized in [21, 23]. The neutrino event rate is approximately proportional to the total proton beam power (energy times current) incident on the target. The parameters for target design to be considered for a given power level are proton energy, pulse duration, and repetition rate. In addition to these the shape and size of the beam spot on the target, and the angle of incidence could also be varied. Studies over the last few years have come to the acceptance that with optimal choice of the above variables the upper limit for a solid target operation is $\sim 2 \mathrm{MW}$. For a given accelerator facility these parameters tend to be correlated and constrained, and therefore a practical limit for a solid target with current technology is probably between 1 and $2 \mathrm{MW}$. Nevertheless, considerable work is needed to achieve a practical design for such a high power solid target and its integration into a focusing horn system. Above 
$2 \mathrm{MW}$, liquid targets are likely the better choice, but these devices will require considerable R\&D and testing before they can be considered practical.

Target R\&D which includes understanding of materials as well as engineering issues of integration is a critical item for the physics program considered in this report.

\section{NEUTRINO BEAM-LINES}

There is currently good experience in building and operating high intensity neutrino beam-lines in the US. The study group has concluded that it is possible to use an existing or build a new super neutrino beam-line based on current technology or extensions of current technology and operate it for the physics program described in this report.

In the following we summarize the status of US high energy accelerator neutrino beam-lines. There are two additional accelerator neutrino beam-lines in the world with comparable technical requirements: the CERN to Gran Sasso neutrino beam which is now operating, and the JPARC to Super-Kamiokande neutrino beam which will start operation in a few years. We will not report on these in this report, but this study included presentations from these facilities. It is clear that there is plenty of communication and shared technical information between these centers and the US.

\subsection{NuMI}

The design and operation of the NuMI beam-line was reported in [23]. In the NuMI beam 120 $\mathrm{GeV}$ protons from the Main Injector, in a single turn extraction of $\sim 10 \mu \mathrm{s}$ duration every $\sim 2 \mathrm{sec}$, are targeted onto a $\sim 94 \mathrm{~cm}$ long graphite target. A conventional 2 horn system is used to charge select and focus the meson beam into a $675 \mathrm{~m}$ long, 2 meter diameter evacuated decay tunnel. The NuMI beam-line is built starting at a depth of $\sim 50 \mathrm{~m}$ and is aimed at a downwards angle of 3.3 deg towards the MINOS detector in Minnesota at a distance of $735 \mathrm{~km}$ from the production target. The flux of the resulting neutrino beam is well known and will be described in a separate section below.

NuMI beam transport, target, horns, and shielding were designed for operation with $4 \times$ $10^{13}$ protons / pulse with a beam power of $400 \mathrm{~kW}$. The goal is to average $3.7 \times 10^{20}$ protons $/$ year . The first year run of NuMI achieved typical beam intensities of $2.5 \times 10^{13}$ protons / pulse or 200 $\mathrm{kW}$. The total integrated exposure was $1.4 \times 10^{20}$ protons on target for the period from March 2005 to March 2006. A number of technical problems were encountered and solved during this time: at the start of the run the cooling water line to the target failed, one of the horns had a ground fault, and most notably a detailed study of the tritium production from the beam-line had to be carried out. Various monitoring systems as well systems to collect tritiated water were installed to elimi- 
nate the amount of tritium going into cooling water and the environment. The experience gained from NuMI operations is indeed invaluable for future operation of neutrino beams.

NuMI beam-line was built at a total cost of \$109M (TEC). The construction time was approximately $\sim 5$ years. The beam-line became operational in March of 2005. Upgrade and operation of the NuMI beam-line for higher intensity for Phase-I in included in the new SNuMI conceptual design report at FNAL[16, 20]. It is anticipated that for operation at $1 \mathrm{MW}$, the primary proton beam-line, the target and horns, and cooling systems in the target hall will require upgrades. New He bags and upgrades to the high radiation work areas will also be installed. The total preliminary cost of this upgrade is estimated to be $\$ 8.7 \mathrm{M}$, which is included in the cost of the proton plan-2 upgrade described in Section 5.

\subsection{Beam towards DUSEL}

Members of this study group [20] have examined the possible siting and construction of a new beam-line towards one of the site candidates for DUSEL, either Henderson mine in Colorado or Homestake mine in South Dakota. The study group has concluded that there are no technical limitations to building such a beam-line on the Fermilab site using the same extraction line from the main injector as the NuMI beam-line. The study group has found significant advantage for lower energy neutrino flux in making the diameter of the decay tunnel for the new beam-line up to 4 meters in diameter.

The new beam-line at FNAL would use the same extraction from the the Main Injector into the NuMI line; a new tunnel would pick up the proton beam from the present tunnel and transport it in the western direction with the same radius of curvature as the Main Injector so that up to 120 $\mathrm{GeV}$ protons can be used with conventional magnets. There is adequate space on the Fermilab site to allow a new target hall with $45 \mathrm{~m}$ length and a decay tunnel of length 400 meter and diameter of 4 meters. This will allow the location of a near detector with $\sim 300$ meters of length from the end of the decay pipe. The new decay pipe would point downwards at an angle of $5.84^{\circ}$ to Homestake (1289km from FNAL) or $6.66^{\circ}$ to Henderson (1495 km from FNAL). The diameter of the decay tunnel is a crucial parameter for both the the neutrino beam intensity and the cost and feasibility of the beam-line; it will require detailed optimization. With our present understanding, construction of a 4 meter diameter decay tunnel with adequate shielding for eventually $2 \mathrm{MW}$ of operation is possible. If the additional concrete shielding is found to be inadequate then the decay pipe would have to be reduced to 3 meter diameter because of the maximum possible span of excavation in the rock under FNAL[24]. The thickness of the shielding has been scaled from the NuMI experience, but the implications of the wider diameter for radiation issues (in particular, tritium production) will need careful study. After careful optimization, the cost of such a project 
can be reliably estimated from the known cost of the NuMI project.

A new beam-line from BNL-AGS to either Homestake $(2540 \mathrm{~km})$ or Henderson $(2770 \mathrm{~km})$ has also been examined in a BNL report[22]. They have made the choice of building the beam-line on a specially constructed hill where the shielded target station is located on top of the hill and the meson decay tunnel is on the downward slope of the hill pointing towards DUSEL at an angle of $11.7^{\circ}$ (Homestake) or $13.0^{\circ}$ (Henderson). Due to the limitations on the height of the hill, the decay tunnel length is restricted to be $\sim 200$ meters with a diameter of 4 meters. The cost of such a beam-line including construction of the hill and proton transport to the top of the hill was estimated to be \$64M (TEC) including contingency and overhead; this cost has been reviewed internally at BNL. Further work on this option has not been part of this study.

\section{EVENT RATE CALCULATIONS}

The neutrino flux and the numbers of expected events with and without oscillations were calculated for both the NuMI off-axis beam and a new broadband beam towards DUSEL. This calculation assumes no detector resolution model or background rejection capability. Both calculations were performed using the same GEANT based GNuMI code. This code has been extensively tested as part of the MINOS collaboration. It has been verified against recent data in the MINOS near detector. The code and associated cross section model is known to produce agreement with the MINOS near detector event rate per proton to about $10 \%$ at the peak of the spectrum and of the order of $20-30 \%$ in the tails of the spectrum with no adjustments. We have also calculated anti-neutrino event rates. The accuracy here is worse simply because of the lack of data from the NuMI beam-line. The anti-neutrino spectra have disagreements between various production codes of $\sim 30 \%$. We believe this is sufficient accuracy for the purposes of this study.

It is very likely that neither the specific off-axis configuration nor the broad-band configuration is highly optimized for the physics under consideration. Such optimization could result in modest gains, especially at low energies. At this stage there is good confidence that the possible improvements will not change the overall picture and sensitivity outlined in this report.

\subsection{NuMI off-axis locations}

We have calculated the neutrino flux and event rates at various off-axis distances from the NuMI beam-line. NuMI was assumed to be configured in the medium energy (ME) or low energy (LE) beam configuration for the results quoted here. The low energy configuration provides better event rate at the $40 \mathrm{~km}$ off-axis location in the low energy peak. There is, however, event rate loss at the $12 \mathrm{~km}$ location. 
The details of the calculation, as well as the spectra are in [5]. Tables II for neutrino running and III for antineutrino running summarizes these event rates. The normalization is per $M W \times 10^{7} \mathrm{sec}$ protons of $120 \mathrm{GeV}$ and for $1 \mathrm{kTon}$ of efficient detector mass. There are no corrections for the type of target nucleus in the detector. There are no efficiencies for reconstruction or fiducial cuts in this calculation.

We have used tabulated cross sections to calculated the event rates in the various columns. The column labeled " $v_{\mu} \mathrm{CC}$ " is the total charged current muon neutrino event rate. " $v_{\mu} \mathrm{CC}$ osc" is the charged current muon neutrino event rate after oscillations. " $v_{e} \mathrm{CC}$ beam" is the charged current rate of electron neutrino contamination in the beam. " $v_{e} \mathrm{QE}$ beam" is the charged current quasielastic event rate of electron neutrino contamination in the beam. "NC- $1 \pi^{0}$ " is the rate of neutral current single pion production integrated over the noted energy range; no detector related rejection is assumed in this table. " $v_{\mu} \rightarrow v_{e} \mathrm{CC}$ " is the charged current event rate of electron neutrinos after oscillations using the oscillations parameters described in Section 4. " $v_{\mu} \rightarrow v_{e}$ QE" is the quasielastic rate of electron neutrinos after oscillations using the oscillations parameters described in Section 4. For example, the total $v_{\mu} \mathrm{CC}$ event rate in 5 years with $1.7 \times 10^{7} \mathrm{sec} / \mathrm{yr}$ in a $100 \mathrm{kton}$ detector without oscillations at $40 \mathrm{~km}$ (LE) off axis can be calculated to be $5.69 \times 100 \times 5 \times 1.7=$ 4836. This event count includes events from both the pion and the kaon peaks at about 0.5 and 4 $\mathrm{GeV}$, respectively.

\subsection{Wide band beam towards DUSEL}

The spectra and the event rate for a beam towards DUSEL were calculated by using the same GNuMI framework but the geometry of the target, horns, and the decay tunnel was changed. The full calculation and the resulting spectra are described in [7]. The integrated event rates are shown in Table IV and Table V. There are a few of comments of importance:

- The calculation in the table is for $1300 \mathrm{~km}$ (the FNAL to Homestake distance), but it could be easily converted to $1500 \mathrm{~km}$ (the distance to Henderson). The UN-oscillated rates scale as $1 / r^{2}$, but the oscillated event rate scale according to the oscillation function. When we demonstrate the full sensitivity calculation later in this report we include the variation with distance. For 1300 versus $1500 \mathrm{~km}$ this variation is small.

- For much of the work on sensitivity we have used the earlier work using a $28 \mathrm{GeV}$ proton beam [12]. The total $v_{\mu} \mathrm{CC}$ event rate in $100 \mathrm{kTon}$ efficient fiducial mass after 5 years at $1.7 \times 10^{7} \mathrm{sec} / \mathrm{yr}$ without oscillations using $E_{p}=28 \mathrm{GeV}$ protons with $1 \mathrm{MW}$ running is 45560 events integrated over 1-20 GeV. It should be kept in mind, however, that according to [17], the available beam power is less for lower energies (see Fig. 7). In the technical 
TABLE II: Signal and background interaction rates for various NuMI beam configurations, baselines and off-axis distances. Rates are given per MW.10 $0^{7}$ s.kT. The rates are integrated over the range 0-20 GeV. For $v_{\mu} \rightarrow v_{e}$ oscillations a value of $\sin ^{2} 2 \theta_{13}=0.04$ and $\Delta m_{31}^{2}=2.5 \times 10^{-3} \mathrm{eV}^{2}$ is used. No detector model is used.

\begin{tabular}{|c|c|c|c|c|c|c|c|}
\hline Distance off-axis & $v_{\mu} \mathrm{CC}$ & $v_{\mu} \mathrm{CC}$ osc & $v_{e} \mathrm{CC}$ beam & $v_{e} \mathrm{QE}$ beam & $\mathrm{NC}-1 \pi^{0}$ & $v_{\mu} \rightarrow v_{e} \mathrm{CC}$ & $v_{\mu} \rightarrow v_{e} \mathrm{QE}$ \\
\hline \multicolumn{8}{|c|}{ NuMI LE tune at $700 \mathrm{~km}$} \\
\hline $0 \mathrm{~km}$ & 400.2 & 267.6 & 4.55 & 0.444 & 21.2 & 3.66 & 0.676 \\
\hline $40 \mathrm{~km}$ & 4.81 & 2.66 & 0.190 & 0.047 & 0.525 & 0.071 & 0.038 \\
\hline \multicolumn{8}{|c|}{ NuMI LE tune at $810 \mathrm{~km}$} \\
\hline $0 \mathrm{~km}$ & 299.0 & 187.4 & 3.40 & 0.332 & 15.8 & 3.10 & 0.551 \\
\hline $6 \mathrm{~km}$ & 198.6 & 107.0 & 2.59 & 0.275 & 11.9 & 2.53 & 0.506 \\
\hline $12 \mathrm{~km}$ & 84.4 & 31.9 & 1.57 & 0.193 & 6.79 & 1.41 & 0.367 \\
\hline $30 \mathrm{~km}$ & 11.6 & 8.38 & 0.353 & 0.070 & 1.32 & 0.107 & 0.046 \\
\hline $40 \mathrm{~km}$ & 5.38 & 2.91 & 0.195 & 0.045 & 0.596 & 0.084 & 0.045 \\
\hline \multicolumn{8}{|c|}{ NuMI ME tune at $810 \mathrm{~km}$} \\
\hline $0 \mathrm{~km}$ & 949.1 & 781.1 & 7.14 & 0.485 & 30.6 & 4.71 & 0.527 \\
\hline $6 \mathrm{~km}$ & 304.9 & 191.4 & 3.83 & 0.313 & 14.9 & 3.19 & 0.491 \\
\hline $12 \mathrm{~km}$ & 80.5 & 32.0 & 1.81 & 0.174 & 5.74 & 1.33 & 0.330 \\
\hline $30 \mathrm{~km}$ & 8.59 & 5.52 & 0.321 & 0.051 & 0.81 & 0.094 & 0.038 \\
\hline $40 \mathrm{~km}$ & 4.14 & 2.40 & 0.168 & 0.032 & 0.427 & 0.054 & 0.022 \\
\hline
\end{tabular}

note [7] it has been shown that the $40-60 \mathrm{GeV}$ spectrum could be very similar to the 28 $\mathrm{GeV}$ with considerable increase in event rate per unit beam power. It has also been shown that it is possible to run at the full energy of $120 \mathrm{GeV}$ and still obtain essentially the same spectrum at the $28 \mathrm{GeV}$ one with a small $0.5^{\circ}$ off-axis angle. With such a choice the neutrino (antineutrino) event rate is 78880 (27030) for $100 \mathrm{kTon}$ and 5 yrs for $1 \mathrm{MW}$ and $1.7 \times 10^{7}$ sec/yr.

- Tables IV and V represent our present understanding of creating such a beam. When optimization is performed coupled to the complete understanding detector performance versus energy, the spectrum could be adjusted to give the best signal/background performance. This could be accomplished by optimizing the horn optics and/or inserting secondary targets (plugs) that remove high energy pions from the beams (see [25]). 
TABLE III: Signal and background interaction rates for various NuMI anti-neutrino beam configurations, baselines and off-axis distances. Rates are given per MW.10 $0^{7}$ s.kT. The rates are integrated over the range 0-20 GeV. For $v_{\mu} \rightarrow v_{e}$ oscillations a value of $\sin ^{2} 2 \theta_{13}=0.04$ and $\Delta m_{31}^{2}=2.5 \times 10^{-3} \mathrm{eV}^{2}$ is used. No detector model is used.

\begin{tabular}{|c|c|c|c|c|c|c|c|}
\hline Distance off-axis & $\bar{v}_{\mu} \mathrm{CC}$ & $\bar{v}_{\mu} \mathrm{CC} \mathrm{osc}$ & $\bar{v}_{e}$ CC beam & $\bar{v}_{e} \mathrm{QE}$ beam & NC- $1 \pi^{0}$ & $\bar{v}_{\mu} \rightarrow \bar{v}_{e} \mathrm{CC}$ & $\bar{v}_{\mu} \rightarrow \bar{v}_{e} \mathrm{QE}$ \\
\hline \multicolumn{8}{|c|}{ NuMI LE tune at $700 \mathrm{~km}$} \\
\hline $0 \mathrm{~km}$ & 157.6 & 102.3 & 1.69 & 0.306 & 19.3 & 1.25 & 0.306 \\
\hline $40 \mathrm{~km}$ & 1.64 & 0.905 & 0.063 & 0.021 & 0.544 & 0.024 & 0.016 \\
\hline \multicolumn{8}{|c|}{ NuMI LE tune at $810 \mathrm{~km}$} \\
\hline $0 \mathrm{~km}$ & 117.7 & 71.0 & 1.26 & 0.229 & 14.4 & 1.026 & 0.285 \\
\hline $6 \mathrm{~km}$ & 77.6 & 39.8 & 0.925 & 0.179 & 10.8 & 0.800 & 0.241 \\
\hline $12 \mathrm{~km}$ & 31.7 & 10.9 & 0.545 & 0.116 & 6.29 & 0.388 & 0.145 \\
\hline $30 \mathrm{~km}$ & 3.87 & 2.69 & 0.122 & 0.035 & 1.31 & 0.043 & 0.025 \\
\hline $40 \mathrm{~km}$ & 1.81 & 0.97 & 0.066 & 0.021 & 0.609 & 0.029 & 0.018 \\
\hline \multicolumn{8}{|c|}{ NuMI ME tune at $810 \mathrm{~km}$} \\
\hline $0 \mathrm{~km}$ & 350.6 & 285.1 & 2.53 & 0.349 & 23.6 & 1.59 & 0.316 \\
\hline $6 \mathrm{~km}$ & 112.8 & 69.0 & 1.28 & 0.208 & 11.9 & 1.011 & 0.259 \\
\hline $12 \mathrm{~km}$ & 27.7 & 9.83 & 0.601 & 0.105 & 4.76 & 0.348 & 0.125 \\
\hline $30 \mathrm{~km}$ & 2.66 & 1.67 & 0.109 & 0.027 & 0.70 & 0.027 & 0.014 \\
\hline $40 \mathrm{~km}$ & 1.27 & 0.73 & 0.057 & 0.016 & 0.376 & 0.015 & 0.008 \\
\hline
\end{tabular}

- We have integrated the rates of various types of events over the same energy interval 0-20 $\mathrm{GeV}$ for Tables II to $\mathrm{V}$. It should be understood that there is considerable variation in the signal to background ratio as a function of energy. To get a full appreciation of this we recommend the reader to explore the spectra at the study web-site [8]. The variation also depends on oscillation parameters. In particular, it should be noted that the $\mathrm{CP}$ violating phase as well as the mass hierarchy is responsible for moving the peak of the oscillation probability by as much as $\sim 0.5(0.7) \mathrm{GeV}$ for the 810 (1300) $\mathrm{km}$ baseline. This variation coupled to the width of the useful spectrum and the detector energy resolution has an impact on the parameter sensitivity of the program. 
TABLE IV: Signal and background interaction rates at $1300 \mathrm{Km}$ (Fermilab-HOMESTAKE) using different WBLE beam energies and off-axis angles. The rates integrated over the neutrino energy range of 0 - 20 GeV. Rates are given per MW.10 ${ }^{7}$ s.kT. For $v_{\mu} \rightarrow v_{e}$ oscillations a value of $\sin ^{2} 2 \theta_{13}=0.04$ and $\Delta m_{31}^{2}=$ $2.5 \times 10^{-3} \mathrm{eV}^{2}$ is used. No detector model is used.

\begin{tabular}{|r|r|l|l|l|l|l|l|}
\hline Degrees off-axis & $v_{\mu} \mathrm{CC}$ & $v_{\mu} \mathrm{CC}$ osc & $v_{e} \mathrm{CC}$ beam & $v_{e}$ QE beam & $\mathrm{NC}-1 \pi^{0}$ & $v_{\mu} \rightarrow v_{e} \mathrm{CC}$ & $v_{\mu} \rightarrow v_{e} \mathrm{QE}$ \\
\hline \hline WBLE $120 \mathrm{GeV}$ at $1300 \mathrm{~km}$ with decay pipe $2 \mathrm{~m}$ radius $380 \mathrm{~m}$ length \\
\hline \hline $0^{\circ}$ & 198.2 & 104.9 & 1.89 & 0.179 & 9.11 & 2.85 & 0.408 \\
$0.5^{\circ}$ & 89.9 & 37.9 & 1.22 & 0.140 & 5.62 & 1.62 & 0.300 \\
$1.0^{\circ}$ & 34.2 & 19.5 & 0.621 & 0.095 & 2.95 & 0.470 & 0.129 \\
$2.5^{\circ}$ & 4.66 & 2.36 & 0.116 & 0.032 & 0.550 & 0.094 & 0.049 \\
\hline \hline WBLE $60 \mathrm{GeV}$ at $1300 \mathrm{~km}$ with decay pipe 2m radius $380 \mathrm{~m}$ length \\
\hline \hline $0^{\circ}$ & 151.0 & 69.2 & 1.34 & 0.169 & 7.83 & 2.53 & 0.403 \\
$0.5^{\circ}$ & 77.2 & 28.7 & 0.906 & 0.134 & 5.33 & 1.52 & 0.305 \\
$1.0^{\circ}$ & 33.3 & 18.4 & 0.520 & 0.098 & 3.08 & 0.480 & 0.141 \\
$2.5^{\circ}$ & 5.05 & 2.56 & 0.120 & 0.035 & 0.611 & 0.105 & 0.058 \\
\hline \hline
\end{tabular}

\section{DETECTOR REQUIREMENTS}

The detector requirements for a detector in a beam towards DUSEL and a detector in the NuMI off-axis beam are quite different. Although the physics goal of measuring $\theta_{13}$, mass hierarchy, and, above all, CP violation is the same, the obstacles to obtain sufficient sensitivity to this physics are very different for the two techniques. We will describe the understanding reached in the process of this study here.

Both techniques are attempting to obtain sensitivity to $\mathrm{CP}$ violation in the neutrino sector by collecting sufficient numbers of $v_{\mu} \rightarrow v_{e}$ appearance events. By obtaining appearance events at difference oscillation phases and energy, matter effects and CP effects can be disentangled to measure oscillation parameters without correlations or ambiguities. Regardless of the technique the most important experimental parameters are the numbers of events at or near the oscillation peaks 
TABLE V: Signal and background anti-neutrino interaction rates at $1300 \mathrm{Km}$ (Fermilab-HOMESTAKE) using different WBLE beam energies and off-axis angles. The rates integrated over the neutrino energy range of 0 - $20 \mathrm{GeV}$. Rates are given per MW.107 s.kT. For $v_{\mu} \rightarrow v_{e}$ oscillations a value of $\sin ^{2} 2 \theta_{13}=0.04$ and $\Delta m_{31}^{2}=2.5 \times 10^{-3} \mathrm{eV}^{2}$ is used. No detector model is used.

\begin{tabular}{|c|c|c|c|c|c|c|c|}
\hline Degrees off-axis & $\bar{v}_{\mu} \mathrm{CC}$ & $\bar{v}_{\mu} \mathrm{CC}$ osc & $\bar{v}_{e}$ CC beam & $\bar{v}_{e} \mathrm{QE}$ beam & $\mathrm{NC}-1 \pi^{0}$ & $\bar{v}_{\mu} \rightarrow \bar{v}_{e} \mathrm{CC}$ & $\bar{v}_{\mu} \rightarrow \bar{v}_{e} \mathrm{QE}$ \\
\hline \multicolumn{8}{|c|}{ WBLE $120 \mathrm{GeV}$ at $1300 \mathrm{~km}$ with decay pipe $2 \mathrm{~m}$ radius $380 \mathrm{~m}$ length } \\
\hline $0^{\circ}$ & 75.0 & 37.7 & 0.570 & 0.106 & 7.79 & 0.669 & 0.160 \\
\hline $0.5^{\circ}$ & 33.5 & 13.0 & 0.356 & 0.077 & 4.90 & 0.332 & 0.103 \\
\hline $1.0^{\circ}$ & 12.0 & 6.47 & 0.185 & 0.056 & 2.64 & 0.122 & 0.056 \\
\hline $2.5^{\circ}$ & 1.41 & 0.694 & 0.037 & 0.013 & 0.499 & 0.033 & 0.022 \\
\hline \multicolumn{8}{|c|}{ WBLE $60 \mathrm{GeV}$ at $1300 \mathrm{~km}$ with decay pipe $2 \mathrm{~m}$ radius $380 \mathrm{~m}$ length } \\
\hline $0^{\circ}$ & 50.5 & 21.3 & 0.373 & 0.088 & 6.05 & 0.507 & 0.137 \\
\hline $0.5^{\circ}$ & 25.4 & 8.52 & 0.248 & 0.066 & 4.23 & 0.272 & 0.094 \\
\hline $1.0^{\circ}$ & 10.3 & 5.38 & 0.144 & 0.045 & 2.52 & 0.116 & 0.058 \\
\hline $2.5^{\circ}$ & 1.36 & 0.667 & 0.031 & 0.013 & 0.518 & 0.035 & 0.024 \\
\hline \multicolumn{8}{|c|}{ WBLE $40 \mathrm{GeV}$ at $1300 \mathrm{~km}$ with decay pipe $2 \mathrm{~m}$ radius $380 \mathrm{~m}$ length } \\
\hline $0^{\circ}$ & 33.8 & 12.5 & 0.270 & 0.069 & 4.70 & 0.366 & 0.110 \\
\hline \multicolumn{8}{|c|}{ WBLE $28 \mathrm{GeV}$ at $1300 \mathrm{~km}$ with decay pipe $2 \mathrm{~m}$ radius $180 \mathrm{~m}$ length } \\
\hline $0^{\circ}$ & 14.6 & 4.94 & 0.076 & 0.026 & 2.64 & 0.172 & 0.065 \\
\hline
\end{tabular}

versus the numbers of irreducible and reducible backgrounds. The numbers of events in either technique are roughly proportional to the exposure defined as the beam power in MW (at some chosen proton energy) times the total detector efficient fiducial size in kTon times the running time in units of $10^{7} \mathrm{sec}$. In the following, to set the rough scale for detectors, we will assume that a few hundred $v_{\mu} \rightarrow v_{e}$ events after accounting for detector efficiency are needed at $\sin ^{2} 2 \theta_{13}=0.1$ per year. As pointed out in section 5, accelerator power of $\sim 1 \mathrm{MW}$ can be obtained and handled with current technology; this sets the scale for the detector size, efficiency, and running times.

\subsection{Off-axis}

In the off-axis technique, we have considered two large detectors at two different locations. On the NuMI beam-line, the places considered for the placement of these detectors are: 1) baseline 
length of $810 \mathrm{~km}$ and off-axis distance of $12 \mathrm{~km}, 2$ ) baseline length of $810 \mathrm{~km}$ and off-axis distance of $40 \mathrm{~km}$. At a length of $810 \mathrm{~km}$ (which is close to the maximum possible on the NuMI baseline), the first and second oscillation maxima for the physics under consideration are at neutrino energy of $1.64 \mathrm{GeV}$ and $0.54 \mathrm{GeV}$, respectively, for $\delta m_{32}^{2}=0.0025 \mathrm{eV}^{2}$. The off-axis distances were chosen to obtain a narrow band neutrino beam at or near these oscillation maxima. These spectra and the event rates can be seen in [5].

Shorter baseline lengths for NuMI off-axis detectors have been considered in the literature [26]. We have commented on this approach as part of the answers to questions in Appendix A. We will not consider this approach here because of the practical difficulties noted.

The main detector requirements for off-axis detectors are:

- Size: To approach the exposure criteria of few hundred events per year for $\sin ^{2} 2 \theta_{13}=0.1$ the total efficient fiducial mass of the detectors at the first and second oscillation maxima needs to be $\sim 100 \mathrm{kT}$. This could be deployed with $50 \mathrm{kT}$ at the first location (12 km off-axis) and $50 \mathrm{kT}$ at the second location (40km off-axis) or all of the mass in one location.

- Cosmic ray rejection: NuMI based off-axis detectors will likely be on the surface or have a small amount of overburden. Surface or near-surface capability is essential for the NuMI based off-axis program because of the geographic nature of the area. As pointed out in Section 12 a surface detector needs to a) have sufficient data acquisition bandwidth to collect all events near the beam spill time, b) eliminate cosmic ray tracks so that the beam events can remain pure, c) tag events due to cosmic rays so that no cosmic ray induced events mimic an in-time beam event. These requirements force the surface detector to be a highly segmented detector with active cosmic ray veto shielding.

- Background rejection: There are two contributions to the background from the neutrino beam: neutral current events and contamination of electron neutrino events. The narrow band nature of the neutrino beam is important for rejection of both of these backgrounds. The neutral current events which tend to have a falling energy distribution can come from both the main peak of the neutrino spectrum and the tails. In the case of location, $40 \mathrm{~km}$ off-axis, the large kaon peak will contribute background. The $v_{e}$ contamination has a broad distribution for both off-axis locations [5]. To use the narrow band nature of the beam effectively to suppress backgrounds, the detector must have the capability to measure neutrino energy (total charged current event energy) with good resolution, which is approximately the same as the width of the narrow band beam. It should also be able to reject $\pi^{0}$ or photon induced showers. 


\subsection{Detectors at DUSEL}

The two sites for DUSEL that made a presentation to this study are 1290 (Homestake) and 1495 (Henderson) km from FNAL. The study has considered distances as far as $\sim 2500 \mathrm{~km}$ and concluded that the physics capability, with some exceptions, is roughly the same for same sized detector. The first and second oscillation maxima for $1290 \mathrm{~km}$ are at $2.6 \mathrm{GeV}$, and $0.87 \mathrm{GeV}$; for $1495 \mathrm{~km}$, they are at $3.0 \mathrm{GeV}$ and $1.0 \mathrm{GeV}$, for $\Delta m_{32}^{2}=0.0025 \mathrm{eV}^{2}$. A new neutrino beam at $0^{\circ}$ or at small off-axis angles has been simulated [7] to show that a spectrum could be made to cover these energies; the critical parameter in the flux at low energies will be the decay tunnel diameter which must be kept to be $\sim 3-4 m$, which is a factor of 1.5-2 larger than the NuMI decay tunnel. The beam-line could be operated at any energy between 30 to $120 \mathrm{GeV}$ proton energy. For higher proton energies work is in progress to remove high energy neutrinos ( $>4 \mathrm{GeV}$ ) that might produce background. The beam-line could also be operated at a slight off-axis angle if the background can be lowered by modest amount while operating at the highest power level possible at $120 \mathrm{GeV}$. For the purposes of setting broad detector requirements we will assume that the spectrum is the similar to Figures 3 or 6.

Detectors at DUSEL (at either Homestake or Henderson) could be placed either on the surface or at a deep site. If placed on the surface the detector considerations would be approximately the same as those for off-axis detectors because the primary design issue would be rejection of cosmic ray background. The availability of deep sites at the appropriate baseline distance for a very large detector are the main reason for locating the detector at DUSEL. Both Henderson and Homestake are planning on large detector caverns at a depth of $\sim 5000 \mathrm{ft}$. We will enumerate the detector requirements assuming this depth.

- Size: To approach the exposure criteria of a few hundred $v_{\mu} \rightarrow v_{e}$ appearance events per year at $\sin ^{2} 2 \theta_{13}=0.1$, the efficient fiducial mass of the detector needs to be $\sim 100 \mathrm{kT}$. In the case of DUSEL all of this mass can be in the same place exposed to a beam that contains both oscillation maxima.

- Cavern: Because of the size required for the detectors, a stable large cavity (or cavities) that can house $\sim 100 k T$ of efficient fiducial mass will be needed. For a water Cherenkov detector, which is well suited for deep operation, the efficiency is expected to be $\sim 25 \%$ indicating a real detector size of several hundred kTon. From preliminary studies it appears that both Henderson and Homestake satisfy this criteria.

- Cosmic ray rejection: Since the cosmic ray rate at the deep sites proposed for DUSEL detectors is very low, it will not be a major factor in detector design. A cosmic ray veto for such 
a detector might be needed for physics other than accelerator neutrino physics; for example, detection of solar neutrinos. But it is not required for the physics discussed here.

- Surface location for a detector: For a liquid argon TPC, the efficiency and background rejection could be high and therefore the detector could be $\sim 100 \mathrm{kt}$. However, for an underground liquid argon TPC the requirements on the cavern will be dominated by safety concerns regarding storage of such a large amount of cryogenic liquid in a deep laboratory. If the liquid argon detector is placed on the surface, the requirements are approximately the same as for the NuMI based off-axis detectors. The dominant requirement will be rejection of cosmic ray background.

- Background rejection: There are two main contributions to the in-time background from the beam: neutral current events, and electron neutrino contamination in the beam. It is expected that the majority of the NC background at low energies will be from single $\pi^{0}$ events that will have to be rejected. In the case of using a wide band beam, there are two tools for signal extraction. Pattern recognition with good capability will be needed to reduce neutral currents, especially single $\pi^{0}$ events. The oscillation pattern in the energy spectrum will also be used to extract the signal. The first oscillation node, in particular, will form a peak above $2 \mathrm{GeV}$ with a well known shape. To allow such a signal extraction, the detector must have good energy resolution for neutrino energy. From the work reported here $\sim 10 \%$ energy resolution above $0.5 \mathrm{GeV}$ including Fermi motion effects will be needed. For a water Cherenkov detector there is new work on pattern recognition to reduce the NC backgrounds and obtain the needed energy resolution. For a liquid argon detector, it has been shown that the NC background can be suppressed to very low levels for low multiplicity events (such as quasi-elastics) while maintaining good resolution.

\section{STATUS OF DETECTOR SIMULATIONS}

\subsection{Water Cherenkov Detector}

As part of this work, we have studied the background rejection and neutrino energy resolution (from charged current events) of a large water Cherenkov detector instrumented in the same manner as Super-Kamiokande. Although considerable further work is needed the capabilities appear to be sufficient for the neutrino oscillation program under consideration. The total mass and exposure needed to achieve good sensitivity to $\mathrm{CP}$ violation in neutrino oscillations was also determined.

The technique of water Cherenkov detectors with non-focusing optics is well understood. In particular, the light yield and the fraction of scattered light can be modeled accurately. Software 
techniques exist that use the pattern of light and the time sequence of photons to reconstruct vertices and trajectories of charged particles. The vertex resolution depends on the timing accuracy of the PMTs. The energy resolution and the energy threshold depends on the total amount of detected light. Both of these have been extensively discussed in technical articles and Ph.D. theses [27, 28]. Considering the substantial existing knowledge and information about this technology, we decided to focus only on the additional new requirements imposed by the accelerator neutrino physics under consideration.

For the program considered here an essential problem is to separate electron shower events from neutral current events, especially events containing a single $\pi^{0}$ in the final state. The goal is to search for $v_{e}$ charged current induced showering events in the 0.5 to $4 \mathrm{GeV}$ range. Single $\pi^{0}$ particles with energies of $1,2,3$ and $4 \mathrm{GeV}$ decay to two photons with a minimum (which is also the most probable) opening angle of $16,8,5$, and 4 degrees, respectively. The probability of a decay with an opening angle of more than $20^{\circ}$ for $1,2,3$ and $4 \mathrm{GeV} \pi^{0}$ 's is $40 \%, 8.2 \%$, $3.6 \%$, and $2.0 \%$, respectively. In a water Cherenkov detector the position where the $\pi^{0}$ photons convert cannot be measured with sufficient precision from the pattern of Cherenkov light, which tends to be two overlapping showering rings. At low $\pi^{0}$ energies the opening angle is sufficiently large compared to the Cherenkov angle $\left(\sim 42^{\circ}\right)$ that single $\pi^{0}$ 's can be separated quite effectively. At energies greater than $2 \mathrm{GeV}$, however, the small angular separation between the two photons makes such separation difficult. It is well known that resonant single pion production in neutrino reactions has a rapidly falling cross section as a function of momentum transfer, $q^{2}$, up to the kinematically allowed value [29]. This characteristic alone suppresses the background by more than 2 orders of magnitude for $\pi^{0}$ (or shower) energies above $2 \mathrm{GeV}$. Therefore a modest $\pi^{0}$ background suppression (by a factor of $\sim 15$ below $2 \mathrm{GeV}$ and $\sim 2$ above $2 \mathrm{GeV}$ ) should make the $\pi^{0}$ background manageable over the entire spectrum.

As part of this study such background suppression has been demonstrated using complete simulation and reconstruction using the Super-Kamiokande detector as the benchmark [30, 31]. Similar suppression has also been obtained independently by another group[32]. In both studies the rejection of backgrounds was enhanced beyond the currently well known capabilities of a SuperKamiokande like detector by using a combined likelihood method. In this method a number of event observables (a complete list can be obtained from the talks in [30] and [32]) with low background discriminating power were combined in a single likelihood cut. The work in [30] chose to cut on this likelihood as a function of reconstructed energy so that the efficiency of this additional cut for charged current electron neutrino events was $\sim 40 \%$. The additional rejection for neutral current events ranges from a factor of 30 at $300 \mathrm{MeV}$ to a factor of 4 at $3 \mathrm{GeV}$ (page 34 in [30]). Table VI shows the rejection power achieved by this method as a function of energy. The table is divided in two parts: before the event energy can be reconstructed the rejection can only be given 
in terms of true simulated quantities such as "true energy". After the event is reconstructed the rejection is given in terms of "reconstructed energy". It should be remarked that the reconstructed energy for an NC event is considerably lower than the true neutrino energy. The total integrated efficiency for signal in this calculation using the $28 \mathrm{GeV}$ spectrum is $37 \%$ from the traditional cuts multiplied by $40 \%$ efficiency of the likelihood cut. As explained in [30] the likelihood cut could be adjusted to have higher efficiency at a cost of higher background. The total integrated rejection of neutral current background is $\sim 13$ for the traditional cuts multiplied by 24.0 using the subsequent likelihood cuts[33].

The work reported in [32] compares her results to [30] (page 31-31 [32]). In the work reported in [32] it was chosen to retain high efficiency (above 70\%) to electron neutrino charged current events; she obtained rejection factors of 10 at $300 \mathrm{MeV}$ declining to 2 at $3 \mathrm{GeV}$. The two calculations are in good agreement if compared at the same efficiency considering that the simulated event sample and methods of discrimination were quite different.

The other important component of this study is the neutrino energy resolution for charged current electron neutrino events. The selection procedure described in the previous paragraphs attempts to select clean events with a single lepton in the final state. To measure the neutrino energy we assume that this selected event is a quasi-elastic scattering event. We then calculate the neutrino energy using the following formula:

$$
E_{\text {rec }}=\frac{2 M_{p} E_{\text {lepton }}-m_{\text {lepton }}^{2}}{2\left(M_{p}-E_{\text {lepton }}+P_{\text {lepton }} \cos \theta_{\text {lepton }}\right)}
$$

to reconstruct the neutrino energy $\left(E_{\text {rec }}\right)$ from the measured electron energy $\left(E_{\text {lepton }}\right)$ and electron angle $\left(\theta_{\text {lepton }}\right)$ with respect to the neutrino direction. The energy resolution using this method has four components. The energy resolution of the electron has been demonstrated to be $4 \%$ at 500 $\mathrm{MeV}$ improving to $2 \%$ above $2 \mathrm{GeV}$ (page 84 in [28]). The angle of the electron with respect to the beam must be measured to calculate the energy of the incoming neutrino. The angular resolution ranges from $3 \mathrm{deg}$ at low energies to $1.5 \mathrm{deg}$ at high energies (page 81 in [28]). The third component to the neutrino energy resolution is the Fermi motion of the struck nucleon inside the oxygen nucleus. This is often modeled using either data from electron scattering or using a simple Fermi gas model. It adds a contribution of $\sim 100-200 \mathrm{MeV}$ to the resolution. Finally, the selected events have a contamination of non-quasielastic events in which the extra particles (such as charged mesons or photons) in the final state are either invisible because they are below Cherenkov threshold or are missed because of poor reconstruction. The final energy resolution including all these effects has been calculated (page 17 in [34]) to be about $\sim 10 \%$ at $1 \mathrm{GeV}$ with significant non-gaussian and asymmetric tails. These tails are due to the nuclear effects and non-quasielastic contributions. The resolution improves at higher energies. The effect of the resolution is that the oscillation pattern remains visible although somewhat degrades. The resolution will have to be 


\begin{tabular}{|l|c|c|c|c|c|c|}
\hline Cut & \multicolumn{5}{|c|}{ Energy Bin (GeV) } \\
& $0-0.5$ & $0.5-1.0$ & $1.0-1.5$ & $1.5-2.0$ & $2.0-3.0$ & $>3$ \\
\hline SK cuts/ $E_{\text {true }}$ & & & & & & \\
$v_{e}$ signal & 0.74 & 0.74 & 0.62 & 0.44 & 0.36 & 0.27 \\
$\mathrm{CC} v_{\mu}$ bkg. & $0.17 \%$ & $0.44 \%$ & $0.75 \%$ & $0.76 \%$ & $0.90 \%$ & $0.45 \%$ \\
$\mathrm{NC}$ bckg. & 0.017 & 0.0443 & 0.053 & 0.070 & 0.077 & 0.086 \\
Beam $v_{e}$ bkg. & 0.86 & 0.75 & 0.57 & 0.46 & 0.36 & 0.23 \\
\hline Likelihood cuts $/ E_{\text {rec }}$ & & & & & & \\
$v_{e}$ signal & 0.40 & 0.40 & 0.40 & 0.39 & 0.40 & 0.40 \\
$\mathrm{CC} v_{\mu}$ bkg. & $6.8 \%$ & $13.6 \%$ & $6.3 \%$ & $8.0 \%$ & $6.5 \%$ & $2.2 \%$ \\
$\mathrm{NC}$ bkg. & $0.72 \%$ & $4.5 \%$ & $6.3 \%$ & $3.9 \%$ & $8.3 \%$ & $7.0 \%$ \\
Beam $v_{e}$ bkg. & 0.37 & 0.41 & 0.40 & 0.37 & 0.39 & 0.34 \\
\hline
\end{tabular}

TABLE VI: Simulation and analysis results on the fraction of events kept after the traditional cuts (top part of the table) and the additional efficiency after the newly developed likelihood cuts (bottom part of the table). The events are divided in 4 parts: signal from $v_{e}$ charged current events (of which a small part are quasi-elastics), charged current $v_{\mu}$ events, neutral current (NC) events, and background due to $v_{e}$ contamination in the beam. There is no entry for background from charged current $v_{\tau}$ because the beam spectrum is dominantly below $\tau$ production threshold $(\sim 3.5 \mathrm{GeV})$ and this background is estimated to be low. The efficiency for the signal and $v_{e}$ background should be the same except for the statistical fluctuations in the Monte Carlo due to small statistics of the $v_{e}$ background. We have retained the numbers in the table to demonstrate consistency.

modeled well to extract the oscillation signal and the oscillation parameters from the far detector data; this is true regardless of the detector type.

The sensitivity calculations described later in this report were performed using the GLoBES framework [82]. For this calculation the detector response was parameterized and adjusted to correspond to the full simulation described above. There are some differences that should be kept in mind to allow comparison between calculations. The first difference is that in the in the sensitivity calculation no events below $0.5 \mathrm{GeV}$ are used. The work in [30] and [32] includes events to lower energies. The second difference is in the energy resolution. The energy resolution obtained after complete simulation and reconstruction is shown in Figure 8 bottom plot. The sensitivity calculation has a parameterized resolution function that includes effects of Fermi motion, resolution on the lepton energy and angle, and non-quasielastic contamination. The two resolution functions 
are shown in Figure 8. The parameterization has somewhat worse resolution in the core than the full simulation, but less tail than the full simulation. The input to the calculation is firmly based on full simulation. But the parameterized background and resolution allows for fast calculation of signal and background rates for different oscillation scenarios; we are also able to change the beam spectrum while keeping the detector performance fixed using this tool.

We note that comparison of signal rates between various authors needs to be done with some care because they may have used different conventions for the sign of the CP violating phase $\delta_{C P}$. The calculation here uses the standard convention from PDG (with $e^{-i \delta}$ in the $U_{e 3}$ matrix element), but some of the simulations reported in the course of this work have the opposite convention with the opposite sign on $\delta$.

We have explored the feasibility of using a water Cherenkov detector for this science with promising results, but considerable further work is needed to optimize the detector and gain complete understanding of its limitations. One question that needs exploration is what are the intrinsic limitations of the water Cherenkov technique in terms of pattern recognition. The literature on the subject is broad and general conclusions can be drawn [36-40]. The question can be quantified in terms of the vertex and angular resolution for single tracks and the ability to separate two tracks that are close in angle and have a common vertex. In the references cited above it has been shown that the photon detection resolution (time and position) in a Super-Kamikande style water Cherenkov detector (proximity focused) does not approach the multiple scattering contribution to that resolution. Therefore, the capabilities of the detector could be improved by modest improvements to the timing and granularity of the PMTs or by addition of ring imaging techniques. In other words, the current capabilities are dominated by the characteristics and geometry of the photo-multiplier array and there is room for improvement.

Photomultiplier coverage and optimization: The optimization of PMT coverage and granularity has not been addressed in our study. This is an appropriate goal for the proponents of the water Cherenkov technique when they write the full proposal. Some hints of the effects of high granularity can, however, be found in the work of Yanagisawa and Dufour. For example, in [30] it is shown that the efficiency for detecting a $\pi^{0}$ particle increases by $20 \%$ as the event moves away from the wall of the detector; this indicates that a larger detector or a detector with more granularity with the same PMT coverage will have better background suppression. In [41], the likelihood based background suppression is shown to have weak dependence on the PMT coverage (either $20 \%$ or $40 \%$ ). These preliminary results indicate that as long as the collected numbers of photoelectrons is reasonably large, the granularity of the PMT readout will have more impact on pattern recognition.

Optimizing beam spectra: In this study we have not made extensive attempts at optimizing the beam spectra versus the detector performance for backgrounds. In [7], a cross section model 

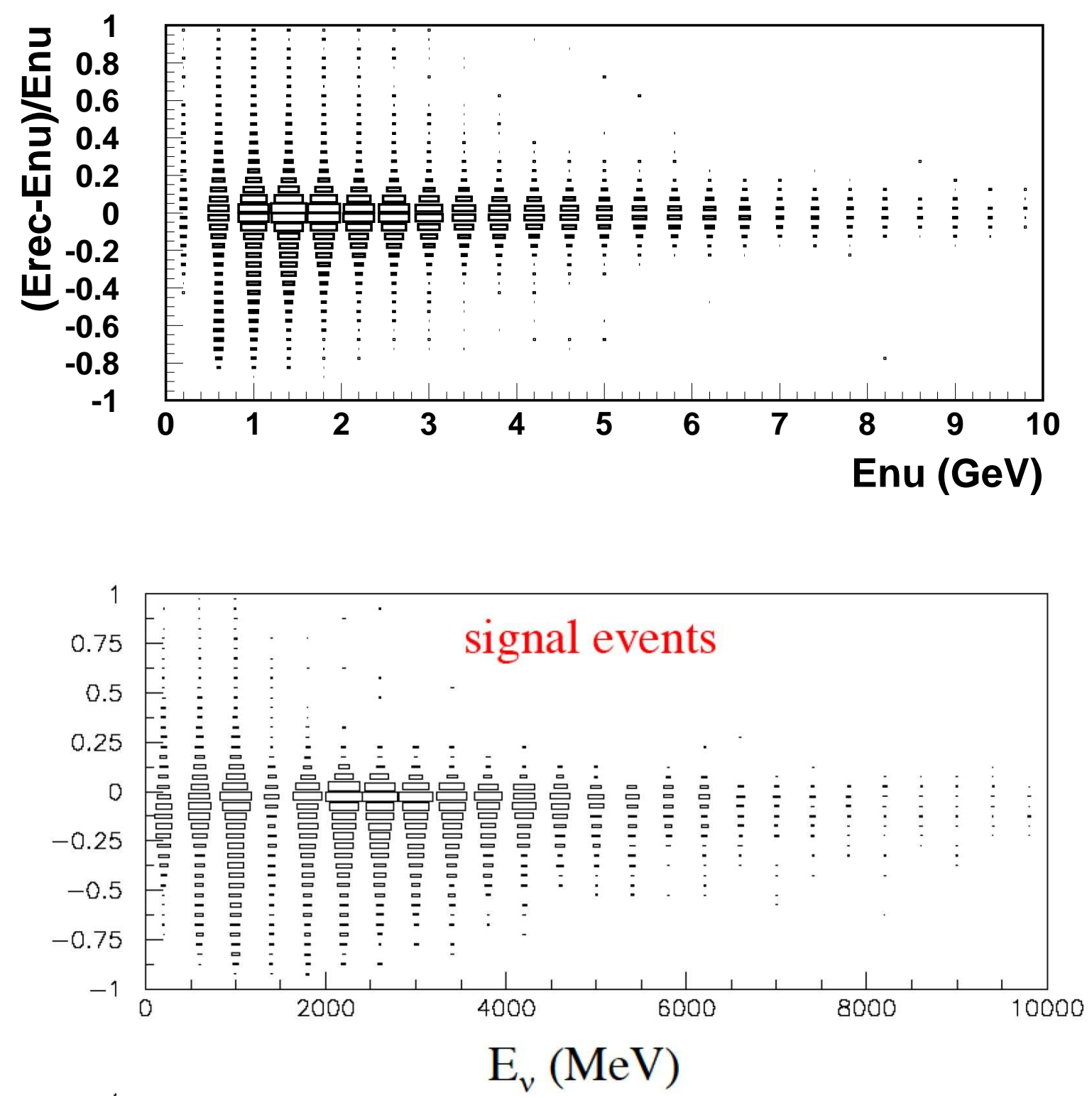

FIG. 8: Top is the parameterized resolution function used in the calculation of sensitivity. Bottom is the energy resolution on selected electron neutrino events after all cuts as described in $[31,34]$. The tail where reconstructed energy is lower than true energy is due to non-quasielastic events that are selected in the selection process. These events have missing particles and therefore have missing energy. The bottom plot was made from simulated events including the effects of oscillations over $1480 \mathrm{~km}$, and therefore there is a depletion of events in the plot around the oscillation minimum. 
was used to calculate signal and background shapes for neutrino spectra produced with different energy proton beams. It is clear that the background will increase for proton energies above 60 $\mathrm{GeV}$ because of the high energy tails. However, for proton energies below $60 \mathrm{GeV}$, the signal versus background performance is approximately constant: there is reduction of background for lower energies, but there is significant statistical gain (for constant power) at higher energies. The intensity at low neutrino energies as well as the elimination of high energy tails will continue to need examination.

There are three ways to optimize the beam spectrum to reduce backgrounds from high energy neutrinos: 1) The optics of the target horn assembly need to be optimized to increase the flux below $3 \mathrm{GeV}$ and reduce it at higher energy, 2) The beam towards DUSEL could certainly be made slightly off-axis. Reference [7] shows that a $0.5^{\circ}$ off-axis angle lowers the event rate $>5 \mathrm{GeV}$ by about a factor of 3 . The $0.5^{0}$ off-axis beam angle can be accommodated in a large 4 meter diameter tunnel, but the implications for shielding, beam dump, as well as future flexibility of the program must be carefully considered. 3) The third option for reducing neutrino flux at higher energies is to introduce a second target (or beam plug) between the two focusing horns. This has been studied for NuMI and found to be effective at reducing tail events by as much as 70\% [42]. Such an option needs careful engineering design because it will affect the radiation environment in the target area.

Near Detector: Lastly, there has been very little study of near detector issues for a beam towards DUSEL. There are 3 issues that need to be discussed. 1) The availability of space: the beam design discussed in Section 7.2 leaves $300 \mathrm{~m}$ of earth shielding from the end of the decay tunnel to a potential near detector site located within FNAL boundaries. The depth at this site would be 192 $\mathrm{m}$ for a beam to Henderson and $176 \mathrm{~m}$ for Homestake. The NuMI near detector is at a depth of 105 meters. The feasibility and cost of a near detector cavern of about 30 meter width/length will need to be examined. 2) The main requirement for the near detector: The most important function of the near detector is the measurement of the neutrino spectrum and backgrounds before oscillations. As explained later for the sensitivity calculation we have assumed that the background will be known to about $10 \%$. This includes the effects of beam and detector modeling as well as nuclear effects which might be different between the near and the far detectors if they are composed of different materials. A harsher requirement on the near versus far energy scale of $<1 \%$ might come from the need to measure $\Delta m_{32}^{2}$ with high precision. 3) The detector granularity, mass, and data acquisition: the event rate at the near site (few events per beam pulse for a $\sim 100$ ton detector) will be much higher compared to the far detector. Reconstruction of these events will likely require a fine grain detector (perhaps a modest sized liquid argon TPC) with electronics that can separate events within the $10 \mu s$ pulse. How the design impacts the requirements outlined above will need to be examined. There is now considerable experience from the NuMI-MINOS on how to use the near detector to perform the appropriate extrapolations from near to far site. From that experience [4] the above 
requirements do not appear to be particularly difficult, but the issue should not be treated cavalierly.

\subsection{Liquid Argon Time Projection Chamber}

Liquid Argon TPC detectors, with fine grained tracking and total absorption calorimetry capability, suggest great promise for sensitivity to long baseline oscillation physics. Hand scanning studies indicate efficiency for charged current quasi-elastic electron neutrino interactions $\left(v_{e} \mathrm{QE}\right)$ greater than $80 \%$ and background rejection of neutral current $\pi^{0}$ events by a factor of 70 [43]. Studies from European groups are consistent with these results [44].

As part of this study, tools have been developed to simulate and reconstruct events in the $\mathrm{E}_{v}=$ 0.5-5 GeV energy region. Studies using these new tools confirm the efficiencies and background rejection from the hand scanning work. Sensitivity calculations folding in these efficiencies, background rejection factors, and resolutions indicate LArTPCs are $\sim 3$ times more sensitive than an equal mass of Water Cherenkov detector 13.

It is primarily the imaging capability that enables LArTPCs to distinguish different event classes from each other. Specifically, while conventional detectors can typically identify only the outgoing lepton in QE interactions, LArTPCs can tag both the outgoing lepton and the recoil proton. Furthermore, a LArTPC can easily and unambiguously identify the interaction point of energetic gamma-rays, for example from $\pi^{0}$ decay, if the separation from the primary vertex is larger than 2 $\mathrm{cm}[45]$.

As part of this study, a GEANT3 simulation of a Liquid Argon TPC was studied and developed to best quantify the detector performance [46]. The Monte Carlo used the NUANCE event generator as input and simulated events in a $7 \times 10 \times 10 \mathrm{~m}^{3}$ box, roughly equivalent to $1 \mathrm{kTon}$. Events are digitized using standard GEANT libraries, and Monte Carlo truth studies performed on this output. Given the imaging capability of a LArTPC, this is an acceptable approximation of an actual event. The criteria to tag a $v_{e} \mathrm{QE}$ interaction are first to see an electron shower as distinct from a muon track. This is assumed to be $100 \%$ efficient. The second criteria is to see a recoil proton coming from the same vertex as the electron. The well established low energy threshold for this is a proton with kinetic energy $>40 \mathrm{MeV}$ [47]. Imposing this requirement, the efficiency for $v_{e} \mathrm{QE}$ events is $>90 \%$. As these are first pass studies, we default to the more conservative $80 \%$ efficiency determination from the hand scanning. Neutral current $\pi^{0}$ backgrounds, with subsequent $\pi^{0} \rightarrow \gamma \gamma$, arise from both $v_{\mu} n \rightarrow v_{\mu} n \pi^{0}$ and $v_{\mu} p \rightarrow v_{\mu} p \pi^{0}$ interactions. The first, $v_{\mu} n \rightarrow v_{\mu} n \pi^{0}$, is rejected because of the lack of any recoil proton. The second, $v_{\mu} p \rightarrow v_{\mu} p \pi^{0}$, is tagged by observation of a $2 \mathrm{~cm}$ or larger gap between the vertex of the recoil proton and at least one of the gammas from the decaying $\pi^{0}$ which converts into an $e^{+} e^{-}$shower. Combining these requirements, only $0.5 \%$ of the NC $\pi^{0}$ backgrounds are not rejected. Further rejection factors are expected by looking at the 
energy deposited in the first few $\mathrm{cm}$ of tracks initiating electron showers versus gamma showers. The overlapping $e^{+} e^{-}$from the gamma shower deposits twice the energy at the beginning of the track as the single electron.

These studies have been performed using the WBLE flux generated with $40 \mathrm{GeV}$ protons used in this study. Full study is needed to understand reconstruction for the other beam option and the NuMI option. Nevertheless, these results are relevant across a broad range of energies. In particular, for $\mathrm{NC} \pi^{0}$ rejection, the separation between the primary vertex and the closest gamma conversion point is roughly independent of the incoming neutrino energy [45]. High multiplicity events in the deep inelastic scattering (DIS) region may be very challenging to reconstruct. Efficiency and background rejection for DIS events for the different flux configurations is also needed.

Advances in automated reconstruction were also pursued as part of this study. The Hough transform based fit algorithm was designed to reconstruct linear tracks from a quasi-elastic event through a parameterization by angle. It efficiently identifies both primary and secondary vertices and reconstructs tracks with resolution of $\sim 2^{\circ}$ (RMS) [45, 48]. This fitter suffices for events with linear tracks and low multiplicity such as quasi-elastics and resonance events. A study of the capability to automatically identify and reconstruct electromagnetic showers is in the early stages.

In the future, this simulation and reconstruction package can be used to study energy resolution for different classes of events.

For the results in this report, we use the energy resolution from previous work. For QE events, a 5\% energy resolution was assumed. This is valid down to $\sim 1 \mathrm{GeV}$, below which few events contribute to the oscillation signals. For non-QE events, a $20 \%$ neutrino energy resolution was assumed. This is likely too conservative in the resonance region where low multiplicity events can still be well measured by LArTPCs, but likely too optimistic for DIS events above 2-3 GeV. Understanding these resolutions as a function of energy is part of the ongoing program of simulation and reconstruction studies.

\section{STATUS OF DETECTOR DESIGN AND TECHNOLOGY}

\subsection{Water Cherenkov conceptual Design}

The water Cherenkov detectors discussed in this study were largely conventional based on the well known technology developed and perfected over the last three decades. The main difference is the factor of $\sim 10$ increase in fiducial mass compared to the largest existing detector (SuperKamiokande). This large increase can be accomplished either by increasing the size of the detector

or by building several detectors (or both). The second important parameter for this detector is the number and size of the photo-multiplier tubes (PMTs). 
Two conceptual designs were reported for this study. They were specifically for the two possible DUSEL locations of Homestake or Henderson, but the authors have acknowledged that their ideas could be adapted to either site with appropriate considerations for site dependent cost factors.

The design reported for the Henderson site (UNO [49]) has a single cavity of dimension $60 \mathrm{~m}$ wide, $60 \mathrm{~m}$ high, and $180 \mathrm{~m}$ long. The 180 meter length is divided in 3 sections. Each section is a separate optical volume with photo-cathode coverage of $10 \%$ for the end sections and $40 \%$ for the central section using the 20 inch diameter PMT developed by Hamamatsu. Each section has fiducial volume (depending on specific physics cuts) of about $150 \mathrm{kTon}$. The depth of the detector in Henderson will be approximately $5000 \mathrm{ft}$.

The design reported for Homestake houses the detector at $4850 \mathrm{ft}$ depth in 3 separate large caverns or modules [50]. The size of the caverns will be in cylindrical with diameter/height of $\sim 53$ meters. The location is at the $4850 \mathrm{ft}$ level of Homestake which is proposed as the Early Implementation Plan for the Homestake lab. The collaboration proposes that the same level be used to accommodate several more cavities to take the total detector mass to megaton over a long period, but the baseline detector is 3 modules. The exact dimensions of the cavities will be determined by the need to maintain fiducial mass of $100 \mathrm{kTon}$ for accelerator neutrino events. Each detector will be instrumented by 10 to 13 inch diameter PMTs with photo-cathode coverage of 25\%. At this stage of simulation and understanding of PMT performance, the Homestake proponents consider the choice of smaller but larger numbers of tubes for granularity adequate for reconstructing the accelerator neutrino events. The concept for the Homestake detector including the physics and a rough estimate for the cost was presented to a program committee for the Homestake interim laboratory. The review can be obtained at [51].

The main concerns for both designs is the cost and time required to build stable and safe cavern(s)[52] and the manufacturing of the necessary number of photo-multiplier tubes.

For the single cavity (UNO) concept an estimate based on the cost of Super-Kamiokande has been made for the cavity excavation of $\$ 168 \mathrm{M}$; the engineering and stability of the cavity needs detailed examination. The total cost including 56000 large 20 inch PMTs and 15000 smaller 8 inch tubes for outer veto volume was estimated to be $\$ 437 \mathrm{M}$. The total construction time will be approximately 10 yrs dominated by the PMT manufacturing time[49].

For the multi-cavity Homestake design, the proponents have performed an initial engineering design for the cavity construction and a stability study[53]. The cost for constructing 3 cavities is estimated to be approximately $\$ 70 \mathrm{M}$ which includes contingency factors. The time scale for constructing the first cavern is 4 yrs and each additional cavern is readied 6 months after the completion of the previous one. The total cost including approximately 50000 PMTs for each detector module is $\$ 309 \mathrm{M}$. The impact on this cost if the size of the module is increased for additional fiducial volume is explained in Appendix A. Based on the Super-Kamiokande experience, the installation 
time for the PMTs is assumed to be about $1 \mathrm{yr}$.

The largest unknown at present for both designs is the schedule for manufacturing the large numbers of PMTs. For the 20 inch PMT option, there appears to be only one vendor at present with a labor intensive manual process. For the smaller PMTs there could be multiple vendors with more automated manufacturing processes. We will comment on this issue again at the end of the report.

There are other technical concerns for such a large water Cherenkov detector: the handling, temperature and purification of such a large amount of water, the engineering for mounting the PMTs and the cabling of the large number of channels, maintenance of the PMTs and associated electronics, and the radiation environment in the deep site which can affect the data rate and the energy threshold of the detector. There is no detailed engineering design for these items, however these issues have been examined by previous generations of these types of detectors. Based on that previous experience both detector designs have included approximate costs in their estimates.

\subsection{Liquid Argon TPC Conceptual Design}

While LArTPCs show great promise with excellent efficiencies and background rejection for a variety of physics goals, they have not yet been demonstrated on scales larger than few hundred tons in size. An active R\&D program culminating in the T600 program [54] has illustrated the capabilities of the detector, however, further R\&D is necessary to consider massive detectors, on the scale of tens of ktons.

There are several different design ideas for massive detectors including a modularized detector [55], a single detector but with modularized drift regions [43], and a single open volume, very long drift detector combining charge and light collection [44]. The technical issues described here are relevant primarily for single massive detectors with modularized drift regions, the design studied by the contributors to this study. For these, there are no major obstacles to scaling to detectors on the scale of 50-100 kTon, however, there is an R\&D path that must be realized in order to consider massive detector construction, operation, and data analysis. Details of this path and major R\&D goals can be found in [56]. The major challenges for scaling to a large detector include:

- Argon purity

- Signal to noise in a massive TPC

- Understanding Cost and Schedule

Progress and path for each of these is described below. 
For ionization electrons to drift $3 \mathrm{~m}$ in a LArTPC, 10ms electron lifetime must be achieved and maintained. Studies from the T600 run suggest this is possible, but, for a massive detector, modifications must be made to the purification system, and the ability to reach purity levels necessary in an industrial environment must be demonstrated. Over the past year, Fermilab has embarked on purity testing towards this goal. They have developed a new non-proprietary Trigon filter (unlike those used in the T600) that can be regenerated in-line. With this filter system, Fermilab has achieved $12 \mathrm{~ms}$ lifetimes in a small test vessel. Over the next year, purity studies will continue with a materials test stand [57] at Fermilab where argon will be re-purified after being exposed to contaminants expected in a massive LArTPC. An additional challenge to purity is a consequence of the inability to achieve vacuum before the initial argon fill in a massive detector. An idea to purge the vessel with clean argon gas prior to liquid fill is being tested at Fermilab now with studies continuing in the upcoming year [58].

A very massive detector will have signal wires as long as tens of meters. Long wires present challenges related to wire breakage, wire assembly and stringing, and electronics noise. Existing R\&D work at Fermilab focuses on assembly techniques and noise pickup using a long wire test stand [59]. Work on electronics design to maximize signal to noise specifically by employing cold electronics, is underway at Fermilab and Michigan State University. A new idea for internal wire configuration, a cellular design, avoids many of the stringing and assembly problems of long wires by stringing wires onto pre-assembled ladders before installation[60].

There are two cost drivers for a liquid argon TPC which have some certainty at this point. The first of these was given by the LArTPC group in its September 2005 report to NuSAG[43]. There, the cost of liquid argon alone (without a purification system) is reported as about $\$ 1 \mathrm{M}$ per kton. Subsequent to that report, a simple scaling relationship has been developed based on information from two vendors for tanks appropriate for containing liquid argon (but without modifications required to put a TPC inside it). This relationship, which is expected to be valid between $5 \mathrm{kTon}$ and $50 \mathrm{kTon}$, is $\$ 2.72 \mathrm{M}+0.306 \$ \mathrm{M} / \mathrm{kTon}$. Thus taking a $50 \mathrm{kTon}$ detector as an example, these two cost drivers (the liquid argon plus a containment tank) would cost about $\$ 50 M+\$ 18 M=\$ 68 M$.

There are many other costs, both technically driven and project driven, but the design of the TPC itself needs to be specified in more detail before such a complete costing exercise can converge. For example, the recently developed cellular design for the TPC significantly changes the requirements on the containment tank compared to the design in the September 2005 LArTPC report to NuSAG. Since this design allows for fabrication of the TPC wire planes at the same time as the containment tank is being constructed, the schedule for construction of the detector is shorter. If electronics are used in the liquid argon, the cellular design will change and the requirements on pattern recognition will become easier. Finally, the idea of using several smaller tanks to achieve a large mass will impact the cost of the purification system as well as the cost of the containment tank(s). These are 
some of the design choices for the TPC that need to be made before a cost estimate of the technical components, other than the liquid argon and the cost for a single containment tank, can be made.

In addition to the major challenges for scaling to large detectors as described above, issues relating to detector siting have been studied. Water Cherenkov detectors must be located deep underground due to cosmic ray backgrounds. By contrast, liquid argon detectors could be located on or near the surface. As part of this study, cosmic rates in a massive LArTPC detector were calculated and their impact on the physics program was considered [61] and is discussed in more detail in Section 12. If massive LArTPCs are sited with some overburden, such as at the $300 \mathrm{ft}$ drive-in site at Homestake, cavern construction for these detectors must still be understood. As part of this study, cavern designs modeled after liquefied natural gas vessels built within ships hulls were considered [62]. This design is promising and studies on this are ongoing.

The R\&D path towards a massive detector includes small scale tests and studies as described above. Construction of a significantly larger prototype, $\sim 1 \mathrm{kTon}$, is necessary before embarking on the massive detector project. The details of this R\&D path at Fermilab will be addressed within the next year.

\section{OVERBURDEN AND SHIELDING}

In this section we briefly discuss the overburden issue in the context of accelerator neutrinos. For non-accelerator physics the issue is discussed in Section 14.

In summary, the background rates in a large detector due to cosmic rays have been calculated for both surface and underground locations. A preliminary evaluation of the consequences for both data acquisition and background to accelerator neutrino events suggests: 1) It is not possible to operate a water Cherenkov detector of size $>50 k T$ on the surface. 2) A fine grained tracking detector such as a liquid argon TPC could be operated on the surface to take data within the short ( $\sim 10 \mu S$ at FNAL Main Injector, $\sim 2.5 \mu S$ for BNL AGS) accelerator spill[61], however background rejection of $\sim 10^{8}\left(\sim 10^{3}-10^{4}\right)$ will be needed against cosmic muons (photons) by either active veto or pattern recognition to reduce the background rate to acceptable levels; this rejection is in addition to the rejection obtained by the timing requirement. We provide a few more details of the calculations below.

A cylindrical tank of size $50 \mathrm{~m}$ height/diameter (approximately 100kT of water) will have a rate of cosmic muons (with momentum $>0.5 \mathrm{GeV} / \mathrm{c}$ ) $250 \mathrm{kHz}$ from the top and $250 \mathrm{kHz}$ from the sides. For a $10 \mu \mathrm{s}$ beam spill this corresponds to 5 muon tracks in the detector. For a single volume water Cherenkov detector in which the photo-multipliers are mounted on the walls looking inwards, each muon on the average will produce a hit in more than $50 \%$ of the PMTs. Therefore, each cosmic ray should be assumed to deaden the entire detector for a period of time which is 


\begin{tabular}{rr} 
Intime cosmics/yr & Depth (mwe) \\
\hline $5 \times 10^{7}$ & 0 \\
4230 & 1050 \\
462 & 2000 \\
77 & 3000 \\
15 & 4400 \\
\hline
\end{tabular}

TABLE VII: Number of cosmic ray muons in a $50 \mathrm{~m}$ height/diameter detector in a $10 \mu \mathrm{s}$ pulse for $10^{7}$ pulses, corresponding to approximately 1 year of running, versus depth in meters water equivalent.

dependent on the dwell time of the muon track and the light inside the detector, the pulse shapes from PMTs, and the data acquisition electronics. All the effects are of order $1 \mu \mathrm{s}$ and therefore make the detector unworkable at the surface. For example, for a detector similar in technology to Super-Kamiokande, the dead-time from the above event rates will exceed 50\% [27]. To reduce this dead-time using fast pulse digitizers is costly, and requires significant software and hardware R\&D to resolve overlapping pulses to reconstruct events with contained vertices. The consequences on background rejection and resolution are at present unknown. The depth required to reduce the number of in-time cosmics to various levels is given in Table VII. A depth of at least $\sim 1000$ meters water equivalent is needed to reduce the muon rate to a level comparable to the rate of events from the neutrino beam so that minimal dependence on pattern recognition (and a modest active veto capability) is needed to separate beam related events.

A $50 \mathrm{kT}$ liquid argon TPC can be contained in a cylindrical tank of size $35.5 \mathrm{~m}$ height/diameter; such a detector will have a cosmic ray muon rate of $125 \mathrm{kHz}$ from the top and $125 \mathrm{kHz}$ from the sides. An examination of cosmic rays [61] in a liquid argon TPC has considered their effects on data acquisition and event reconstruction, and as a source of background. The rate of cosmic rays was shown to be tolerable with the proposed drift-time and data acquisition system for cycles up to $5 \mathrm{~Hz}$. In this scheme the detector takes data in a short time interval (currently proposed to be 3 drift times) near the beam time. This is sufficient to cover most possible accelerator cycle times discussed above. The high granularity of the detector should allow removal of cosmic muons from the data introducing a small $(<0.1 \%)$ inefficiency to the active detector volume, so that most of the accelerator induced events are unobscured. If a cosmic ray muon (photon) event mimics a contained in-time neutrino event it must be rejected based on pattern recognition. The rejection required is $\sim 10^{8}$ for muon cosmics and $\sim 10^{3}-10^{4}$ for photon cosmics; given the fine grained nature of the detector this rejection is considered achievable, but still needs to be demonstrated by detailed simulations. 


\section{ANALYSIS OF SENSITIVITY TO OSCILLATION PARAMETERS}

In this section we will combine the information from the previous sections on the intensity of the accelerator beam and detector performance to calculate the sensitivity to oscillation parameters. The main features of the accelerator and detector performance can be summarized as follows:

For the sensitivity calculations we will assume that we can obtain a total of $60 \times 10^{20}$ protons at $120 \mathrm{GeV}$. This total is to be divided between neutrino and anti-neutrino running. To convert this luminosity to running time we will assume that the accelerator can produce proton intensity according to Figure 7 in the accumulator upgrade scenario. In the accumulator upgrade scenario power level of $1.2 \mathrm{MW}$ is expected at $120 \mathrm{GeV}$. This running scenario will be used for both the off-axis and the DUSEL based options for the Phase II program. We will make comments on running at lower proton energies as well as more exposure. The impact on the running time will be according to the power curve in Figure 7. The raw event rates can be obtained from Tables II to V.

For the first DUSEL based calculation we have assumed a water Cherenkov detector with a total fiducial mass of 300 kTon with the performance described in Section 10.1. The calculation was performed with the GLoBES package[35] with the beam spectra and detector performance specified according to the work in this report. For the DUSEL baseline we have performed cal-

culations ranging from $500 \mathrm{~km}$ to $2500 \mathrm{~km}$ with various beam configurations. We cannot display all calculations in this report due to length considerations, but they can be obtained from the study website[8]. Differences in parameter sensitivity due to baseline will be discussed. Most of the calculations shown here will be for the $1300 \mathrm{~km}$ distance.

For the second DUSEL based calculation we have assumed a 100 kTon liquid argon time projection chamber with the performance indicated in section 10.2. Briefly, we assume $80 \%$ efficiency for electron neutrino events with very little background from other sources.

For the off-axis calculations several different combinations were calculated. First, for comparison purposes the calculation is performed for NOvA with the detector performance obtained from the NOvA collaboration. Second, a 100 kTon total mass for a liquid argon detector TPC was assumed for phase II. The performance was evaluated for setting the entire detector mass at the same location as NOvA and also for setting $50 \mathrm{kT}$ at the NOvA site (12 km off-axis) and $50 \mathrm{kT}$ at the site (40 km off-axis) where the second oscillation maximum can be observed. Doubling of the total mass at the two sites was also examined.

Lastly, we note that unless otherwise noted the oscillation parameters used for the calculations are as follows:

$$
\begin{gathered}
\Delta m_{21}^{2}=8.6 \times 10^{-5} \mathrm{eV}^{2} \\
\sin ^{2} 2 \theta_{12}=0.86
\end{gathered}
$$




$$
\begin{gathered}
\Delta m_{32}^{2}=2.7 \times 10^{-3} \mathrm{eV}^{2} \\
\sin ^{2} 2 \theta_{23}=1
\end{gathered}
$$

The above parameters $\theta_{13}, \delta_{C P}$ and the mass hierarchy (normal or reversed) are left free in the calculation.

Before describing the sensitivity to $v_{e}$ appearance, we first make a few comments on the $v_{\mu}$ disappearance measurement. Either of the two experimental concepts (a new beam to a DUSEL location or new off axis detectors on the surface in the NuMI beam-line) have sufficient statistical reach to make a very precise measurement of the atmospheric oscillation parameters $\left(\Delta m_{32}^{2}\right.$ and $\sin ^{2} 2 \theta_{23}$. For a $100 \mathrm{kTon}$ detector, $\sim 10000 v_{\mu}$ CC events per year are expected in either scenario with approximately $1 / 2$ disappearing due to oscillations (see Section 8 for exact numbers for specific beam configurations). The statistical precision after several years of running, therefore, will be $<1 \%$ for both $\Delta m_{32}^{2}$ and $\sin ^{2} 2 \theta_{23}$. A discussion of this measurement for a DUSEL based detector can be seen in [12]. A similar discussion for the off-axis scenario is in [73]. To obtain the best measurement, both the neutrino event energy resolution (including nuclear target effects due to Fermi motion and re-scattering) and the absolute energy scale need to be well modeled. With current knowledge of these limitations the measurement will most likely be systematically limited to about $1 \%$ for both $\Delta m_{32}^{2}$ and $\sin ^{2} 2 \theta_{23}$. There is an important difference between the off-axis measurement and the broad band measurement. The oscillation shape including a nodal pattern if the baseline distance is sufficient can be measured with a DUSEL based detector. Such a measurement will exhibit less correlation between the two parameters $\Delta m_{32}^{2}$, which determines the position of the node in energy, and $\sin ^{2} 2 \theta_{23}$ which determines the depth (or amplitude) of the node. A precise measurement of the shape could also limit non-standard physics models of decay, decoherence, extra-dimensions, etc.

\subsection{Sensitivity of a FNAL to DUSEL based program}

For the calculations reported here we have used the $120 \mathrm{GeV}$ beam with 380 meter decay tunnel with a 0.5 deg off-axis angle. As explained above, the spectrum from such a configuration is well matched to the physics at this current time. The energy of the proton beam and the horn optics need to be optimized further. 


\subsubsection{Water Cherenkov Detector}

The reconstructed electron neutrino spectrum with $300 \mathrm{kT}$ of fiducial mass and a total exposure of $60 \times 10^{20}$ protons (divided equally between neutrinos and antineutrino running) is shown in Figure 9. This spectrum includes effects of nuclear motion, detector resolution, detector signal efficiency, and background rejection using the performance as described in Section 10.1. The plot is made for $\sin ^{2} 2 \theta_{13}=0.04$ and a baseline of $1300 \mathrm{~km}$. The plots for $1480 \mathrm{~km}$ can be obtained from [8]. The left plots are for the normal mass hierarchy $\left(m_{1}<m_{2}<m_{3}\right)$. The right hand plots are for the reversed hierarchy $\left(m_{3}<m_{1}<m 2\right)$. The top plots are for neutrino running and bottom plots are for anti-neutrino running.

By fitting the spectra in Figure 9 we can extract the parameters $\theta_{13}, \delta_{C P}$ and the mass hierarchy. We calculate a $\chi^{2}$ function and extract the confidence levels for a simultaneous fit to these three parameters. For the input values of the other oscillation parameters we assume 1 sigma errors as follows:

$$
\begin{gathered}
\theta_{12}=0.59 \pm 10 \%, \Delta m_{21}^{2}=(0.86 \pm 10 \%) \times 10^{-5} \\
\theta_{23}=\pi / 4 \pm 5 \%, \Delta m_{31}^{2}=(2.7 \pm 5 \%) \times 10^{-3}
\end{gathered}
$$

We also include $5 \%$ error on the matter density. The calculation includes correlations between all parameters and accounts for possible degeneracies. The spectra were fit with statistical errors and with $10 \%$ systematic error on the background and $1 \%$ systematic error on the normalization with no correlations between neutrino and anti-neutrino channels. Details of the analysis method is in [67] where the same analysis was performed with a different spectrum and detector performance.

In Figure 10 we show the confidence level contours for measuring the pair of parameters $\left(\theta_{13}\right.$ and $\delta_{C P}$ ). This calculation was performed for normal mass hierarchy, a baseline of $1300 \mathrm{~km}$, and a total exposure of $60 \times 10^{20}$ protons equally divided between neutrino and anti-neutrino running. The result for $1480 \mathrm{~km}$ is approximately the same. In the case of normal hierarchy, the neutrino data alone can be used to measure the parameters over a large range of parameter space. But if the mass hierarchy is reversed, anti-neutrino data has to be used. The resolution obtained after combining both neutrino and anti-neutrino data (right hand side) is approximately independent of mass hierarchy. From the right hand plot, it is clear that the parameter measurement will suffer from background below $\sin ^{2} 2 \theta_{13}=0.01$, but above this value the resolution on the CP phase of about $\pm 20^{\circ}$ ( 1 sigma) is approximately independent of $\theta_{13}$.

If there is no excess of electron events observed then we can set a limit on the value of $\sin ^{2} 2 \theta_{13}$ as a function of $\delta_{C P}$. Such sensitivity limits are shown in Figure 11. The range of parameters over which the mass hierarchy can be resolved is shown in Figure 12. We have chosen to display the 
limits separately for the two mass hierarchies. Some of the structure in the 3 sigma lines is due to the limited number of bins used in the calculation. The region to the right hand side of each curve excludes the opposite mass hierarchy at the respective confidence level. Similarly the range of parameters over which CP violation can be established (i.e. determine that $\delta_{C P}$ is not 0 or $\pi$ ) is displayed in Figure 13. 

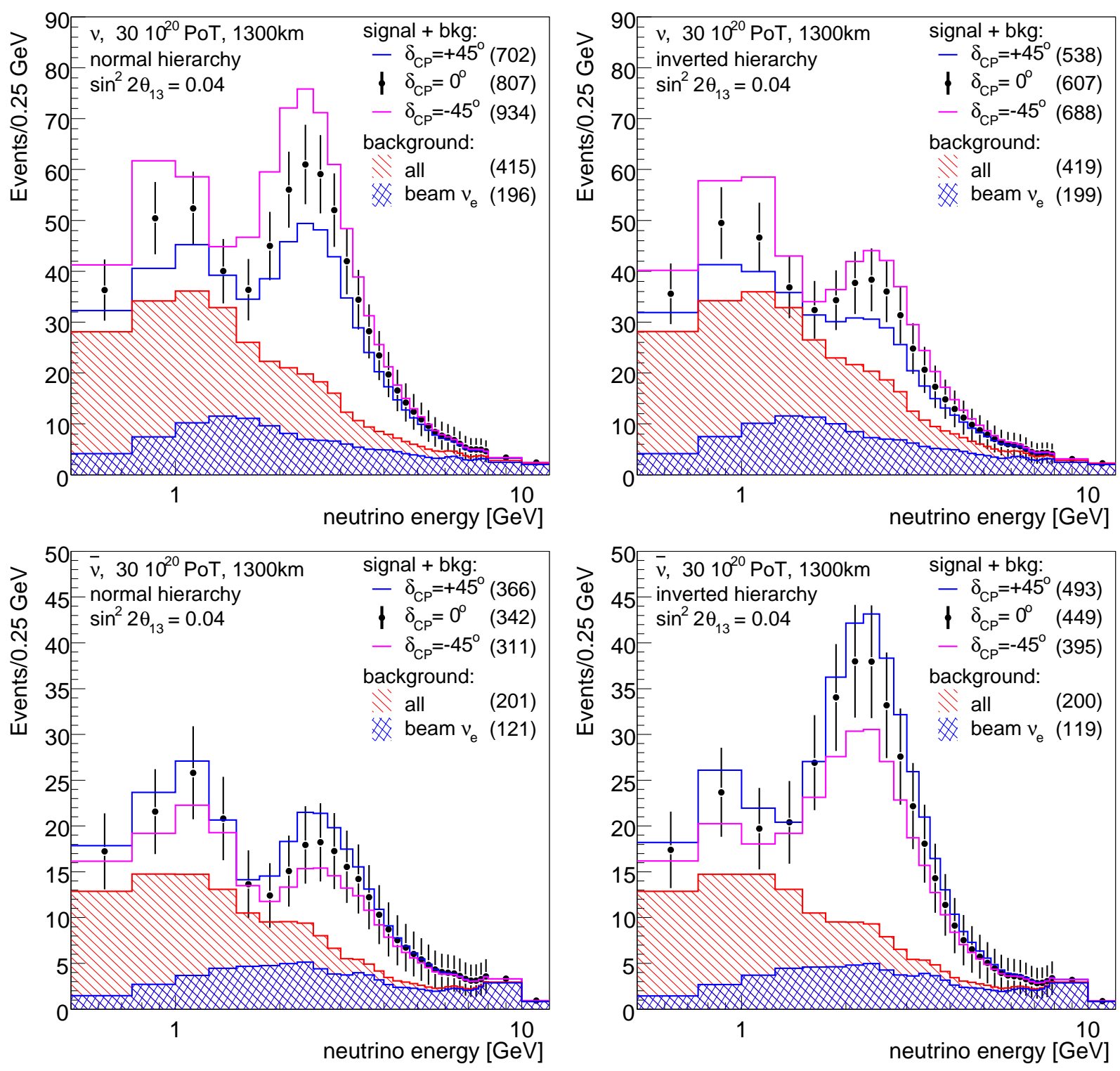

FIG. 9: Simulation of detected electron neutrino (top plots) and anti-neutrino (bottom plots) spectrum (left for normal hierarchy, right for reversed hierarchy) for 3 values of the CP parameter $\delta_{C P},-45^{\circ}, 0^{\circ}$, and $-45^{\circ}$, including background contamination. This simulation is for $300 \mathrm{kT}$ of water Cherenkov detector with the performance described in Section 10.1. This is for a exposure of $30 \times 10^{20}$ POT for each neutrino and anti-neutrino running. The hatched histogram shows the total background. The $v_{e}$ beam background is also shown. The other parameters and running conditions are shown in the figure. 

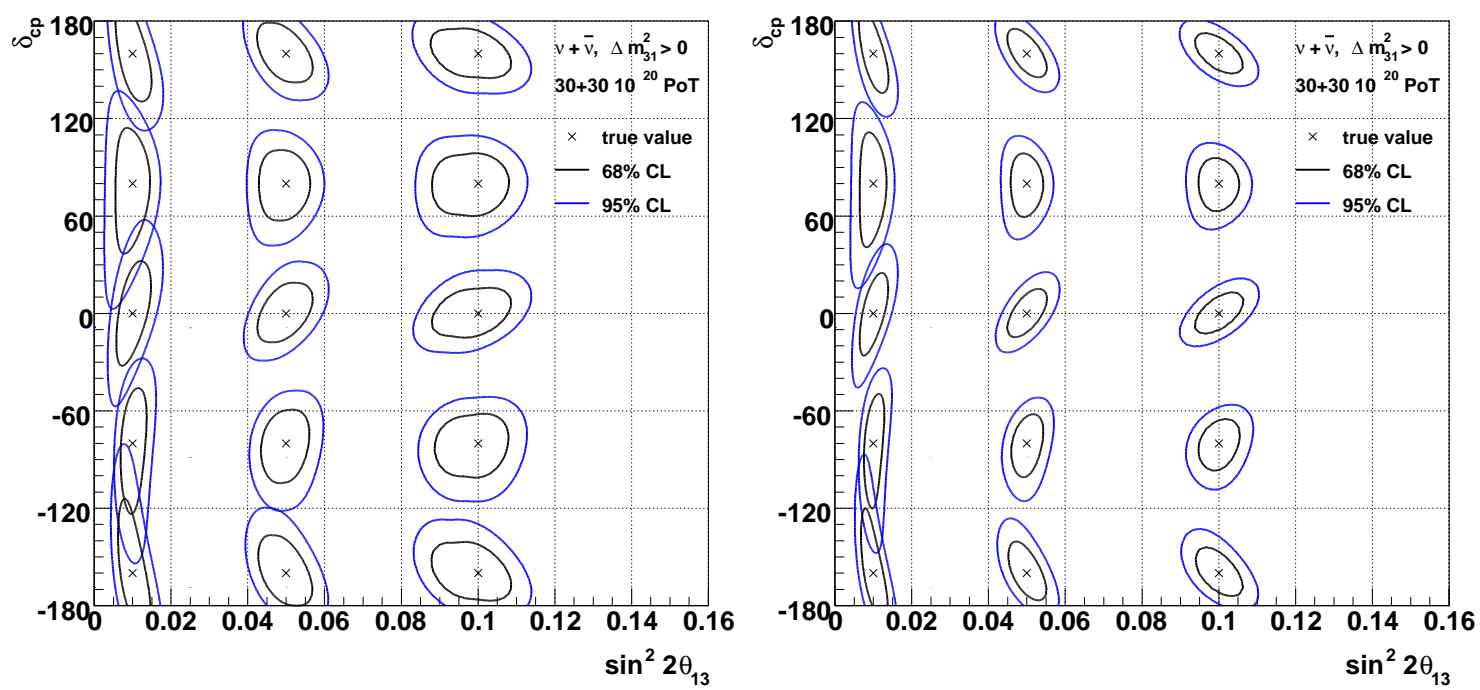

FIG. 10: $90 \%$ and $95 \%$ confidence level error contours in $\sin ^{2} 2 \theta_{13}$ versus $\delta_{C P}$ for statistical and systematic errors (left hand plot) for 15 test points. This is for a $300 \mathrm{kT}$ water Cherenkov detector with a total exposure of $60 \times 10^{20}$ POT. The right hand side is for statistical errors alone. This plot was made for normal mass hierarchy. We assume 10\% systematic errors on the background for this plot.
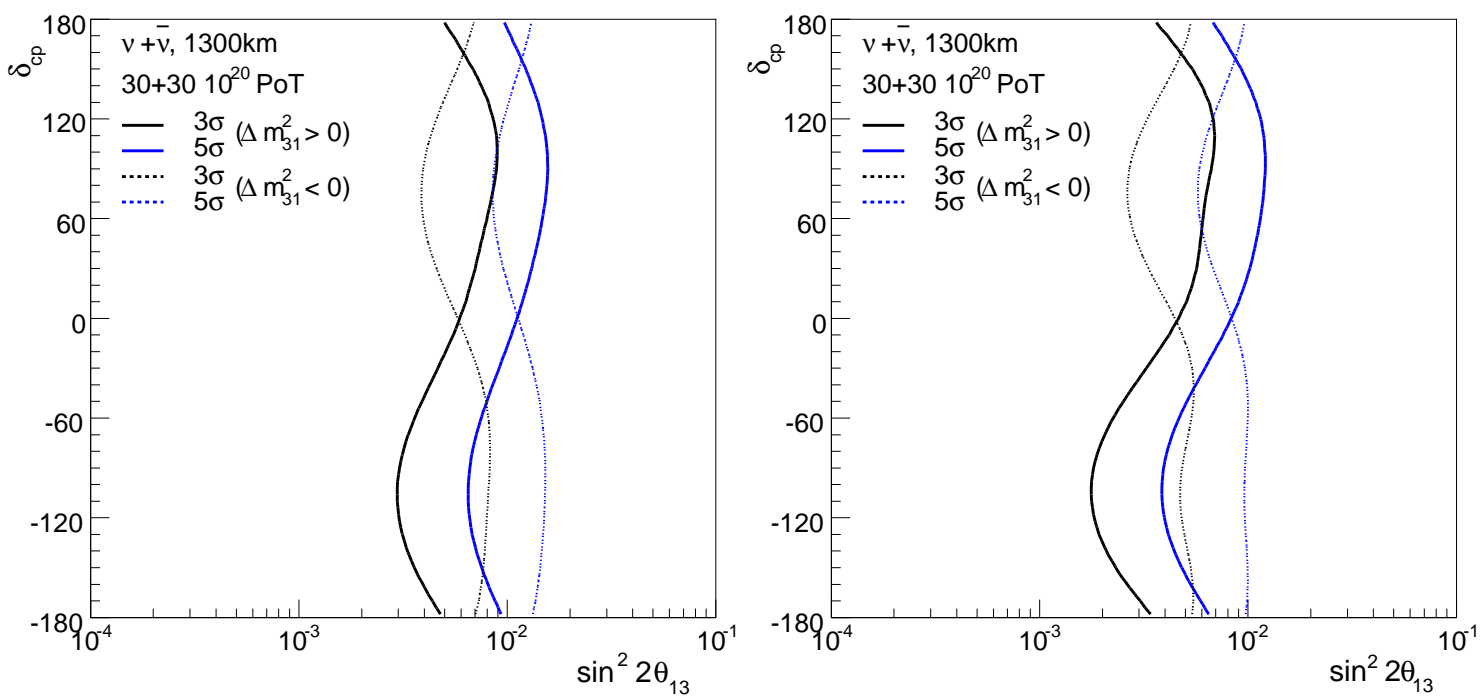

FIG. 11: 3 sigma and 5 sigma confidence level exclusion limits for determining a non-zero value for $\theta_{13}$ in $\sin ^{2} 2 \theta_{13}$ versus $\delta_{C P}$ for statistical and systematic errors (left hand plot). This is for a $300 \mathrm{kT}$ water Cherenkov detector with a total exposure of $60 \times 10^{20}$ POT. The right hand side is for statistical errors alone. The solid (dashed) lines are for normal (reversed) mass ordering. We assume 10\% systematic errors on the background for this plot. 

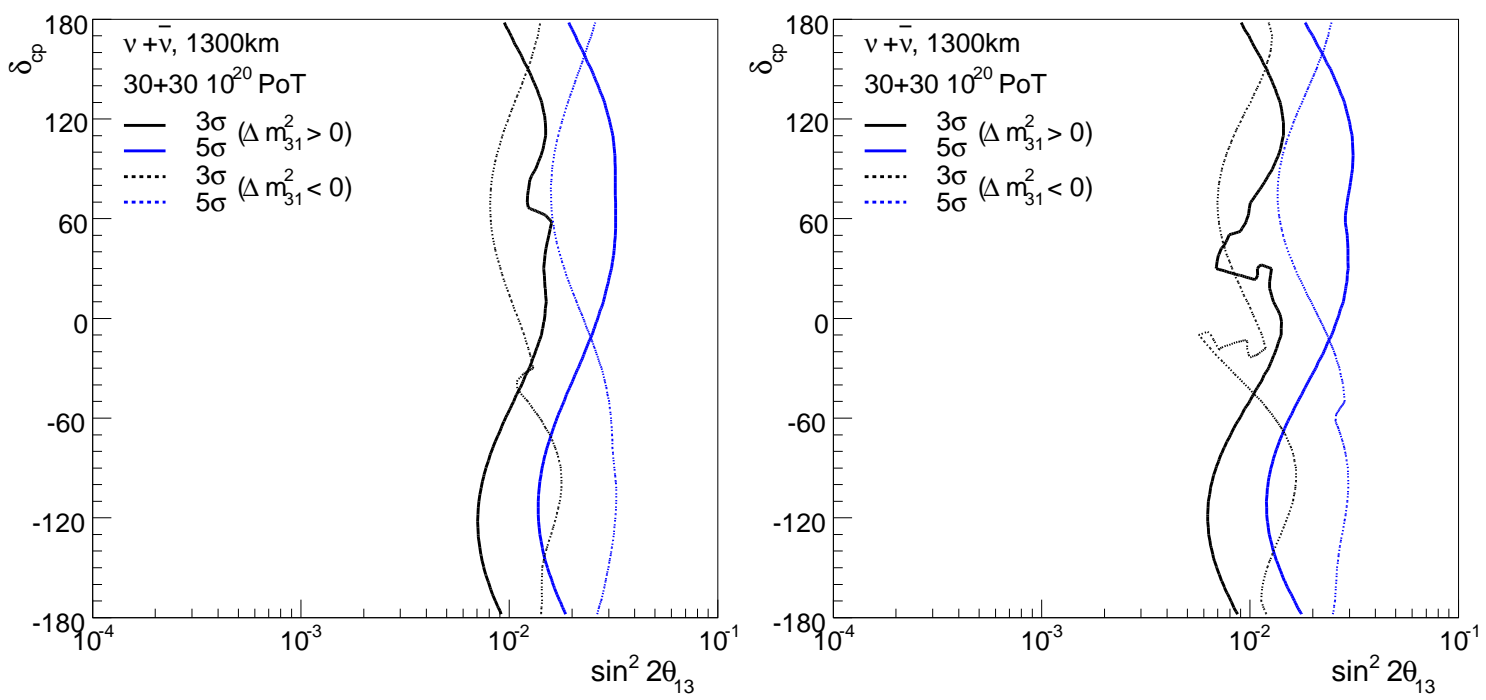

FIG. 12: 3 sigma and 5 sigma confidence level exclusion limits for determining the mass hierarchy in $\sin ^{2} 2 \theta_{13}$ versus $\delta_{C P}$ for statistical and systematic errors (left hand plot). This is for a $300 \mathrm{kT}$ water Cherenkov detector with a total exposure of $60 \times 10^{20}$ POT. The right hand side is for statistical errors alone. The solid (dashed) lines are for normal (reversed) mass ordering. We assume 10\% systematic errors on the background for this plot.
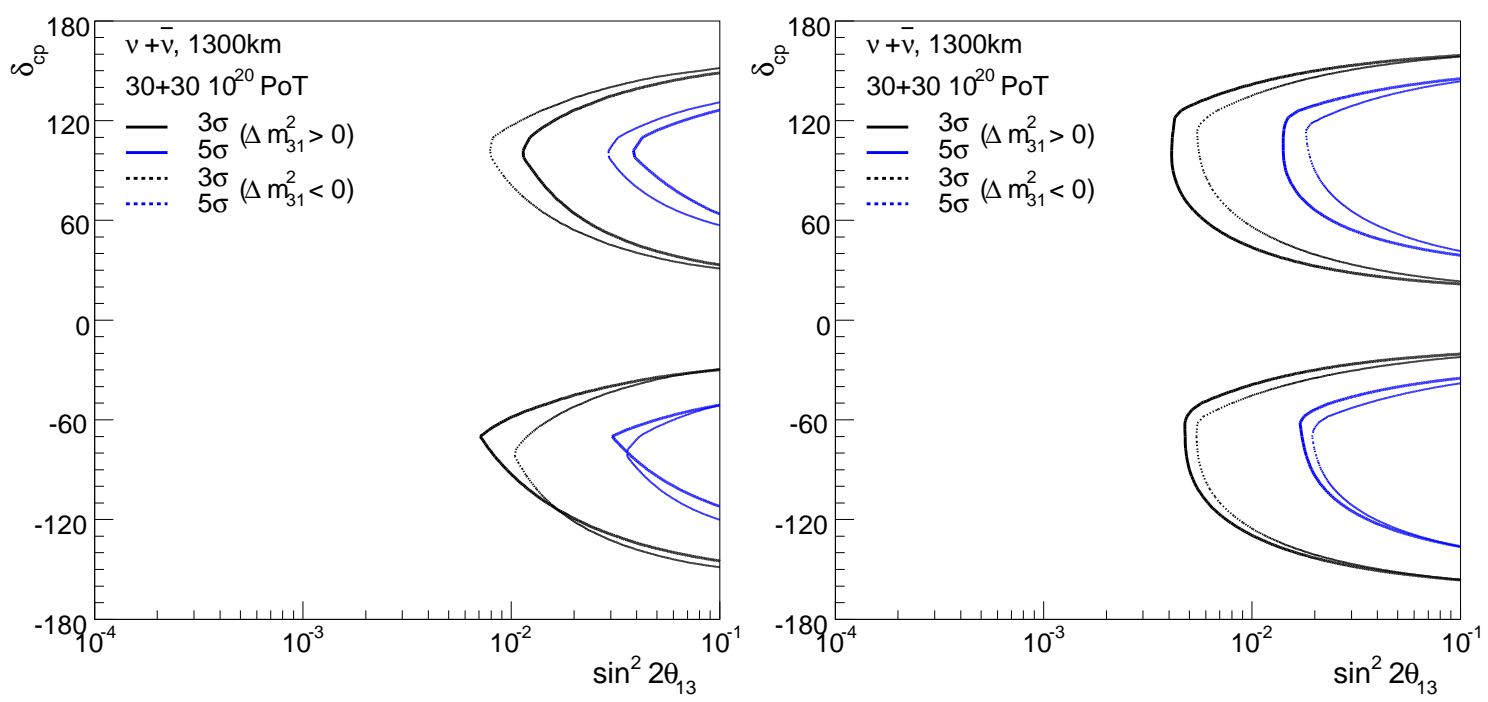

FIG. 13: 3 sigma and 5 sigma confidence level exclusion limits for determining $C P$ violation in $\sin ^{2} 2 \theta_{13}$ versus $\delta_{C P}$ for statistical and systematic errors (left hand plot). This is for a $300 k T$ water Cherenkov detector with a total exposure of $60 \times 10^{20}$ POT. The right hand side is for statistical errors alone. The solid (dashed) lines are for normal (reversed) mass ordering. We assume 10\% systematic errors on the background for this plot. 
Sensitivity variation with exposure: The exposure assumed in the above plots was $30 \times 10^{20}$ protons on target for each neutrino and antineutrino running. This corresponds to 3 years of running for each polarity for $1.2 \mathrm{MW}$ of beam power and $1.7 \times 10^{7} \mathrm{sec}$ of running at $120 \mathrm{GeV}$. If we were to run the antineutrino beam for twice the exposure of neutrino, then the number of events is approximately balanced between $v$ and $\bar{v}$. Such unequal running is advantageous in the case of the reversed hierarchy. An analysis of the total exposure was performed in [67]. It was found that longer exposures will have relatively modest effect on the sensitivity to $\sin ^{2} 2 \theta_{13}$ and the mass hierarchy resolution, but could be important for improving the precision on the $\mathrm{CP}$ violation measurement. Exclusion contours for twice the exposure (total exposure of $120 \times 10^{20}$ protons) are shown in Figures 14 (for determining non-zero $\theta_{13}$ ), 15 (for determining mass hierarchy), and 16 (for determining $\mathrm{CP}$ violation).
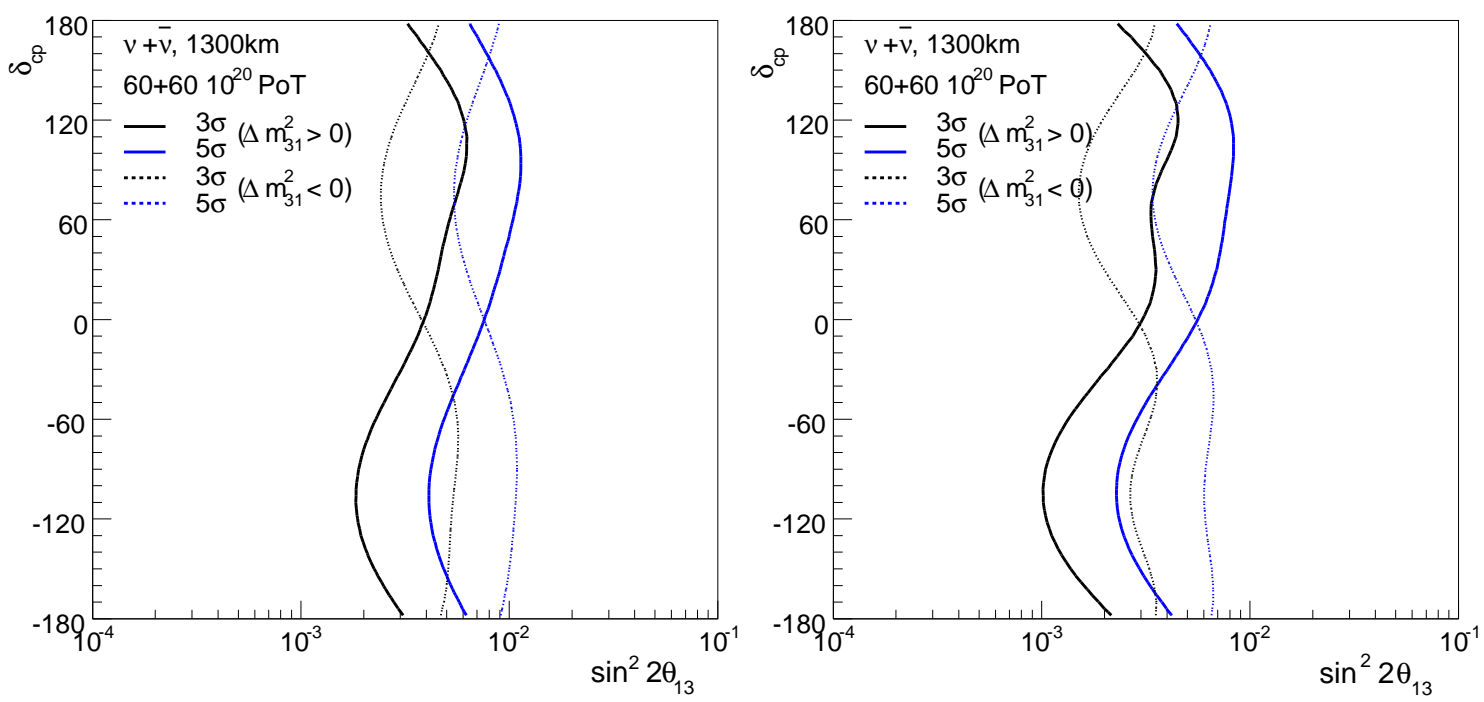

FIG. 14: 3 sigma and 5 sigma confidence level exclusion limits for determining a non-zero value for $\theta_{13}$ in $\sin ^{2} 2 \theta_{13}$ versus $\delta_{C P}$ for statistical and systematic errors (left hand plot). This is for a $300 \mathrm{kT}$ water Cherenkov detector with a total exposure of $120 \times 10^{20}$ POT. The right hand side is for statistical errors alone. The solid (dashed) lines are for normal (reversed) mass ordering. We assume 10\% systematic errors on the background for this plot.

Sensitivity variation with distance: Analysis in [67] showed that there is significant variation in sensitivity up to $1500 \mathrm{~km}$ for determination of the mass hierarchy. This is reproduced in Figure 17. The variation in sensitivity to $\theta_{13}$ was found to be mild partly because of the larger matter enhancements (in neutrino (antineutrino) mode for normal (reversed) mass hierarchy) at longer distances. There is a slow decrease in the sensitivity to CP violation at longer distances, but this is attributed to the shape of the spectrum used for this calculation. At distances below $1000 \mathrm{~km}$, there is a degradation in the $\mathrm{CP}$ sensitivity because of the need to resolve the ambiguity due to the mass 

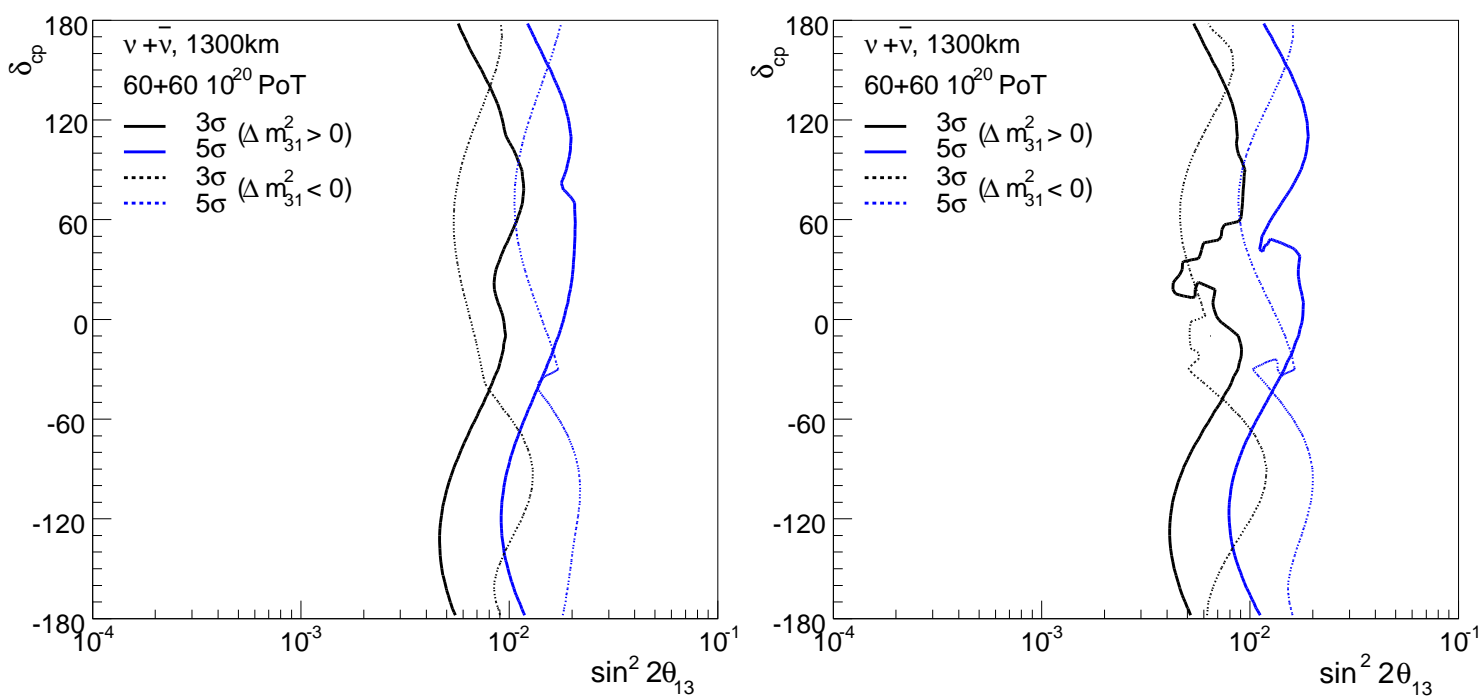

FIG. 15: 3 sigma and 5 sigma confidence level exclusion limits for determining the mass hierarchy in $\sin ^{2} 2 \theta_{13}$ versus $\delta_{C P}$ for statistical and systematic errors (left hand plot). This is for a $300 \mathrm{kT}$ water Cherenkov detector with a total exposure of $120 \times 10^{20}$ POT. The right hand side is for statistical errors alone. The solid (dashed) lines are for normal (reversed) mass ordering. We assume 10\% systematic errors on the background for this plot.

hierarchy with the same data. Complete calculations with same assumptions for detector size and performance and spectra can be obtained from the [8] for distances up to $2600 \mathrm{~km}$; this covers the various options for the DUSEL locations.

\section{Sensitivity variation due to systematics and parameter variation:}

The sensitivity calculation reported in this section follows the prescription from [35, 67]. They include the parameter variation as described above (Section 13.1.1). In addition, we assume a 10\% systematic error on the total background. Considering recent and past experience with background determination in long baseline experiments, the $10 \%$ systematic error is very likely a pessimistic assumption [83, 84], especially with a planned near detector. Sensitivity estimates with other assumptions for the systematic error can be obtained from the website [8]. The conclusion from these studies is that the background systematic error will most likely dominate over the parameter variation. Therefore, in figures 10 to 16 , we have chosen to show the sensitivity with and without systematic errors. Our conclusion is that the wide band technique which leads to a spectrum measurement is robust against parameter changes and background systematic errors over a reasonable range [67]. 

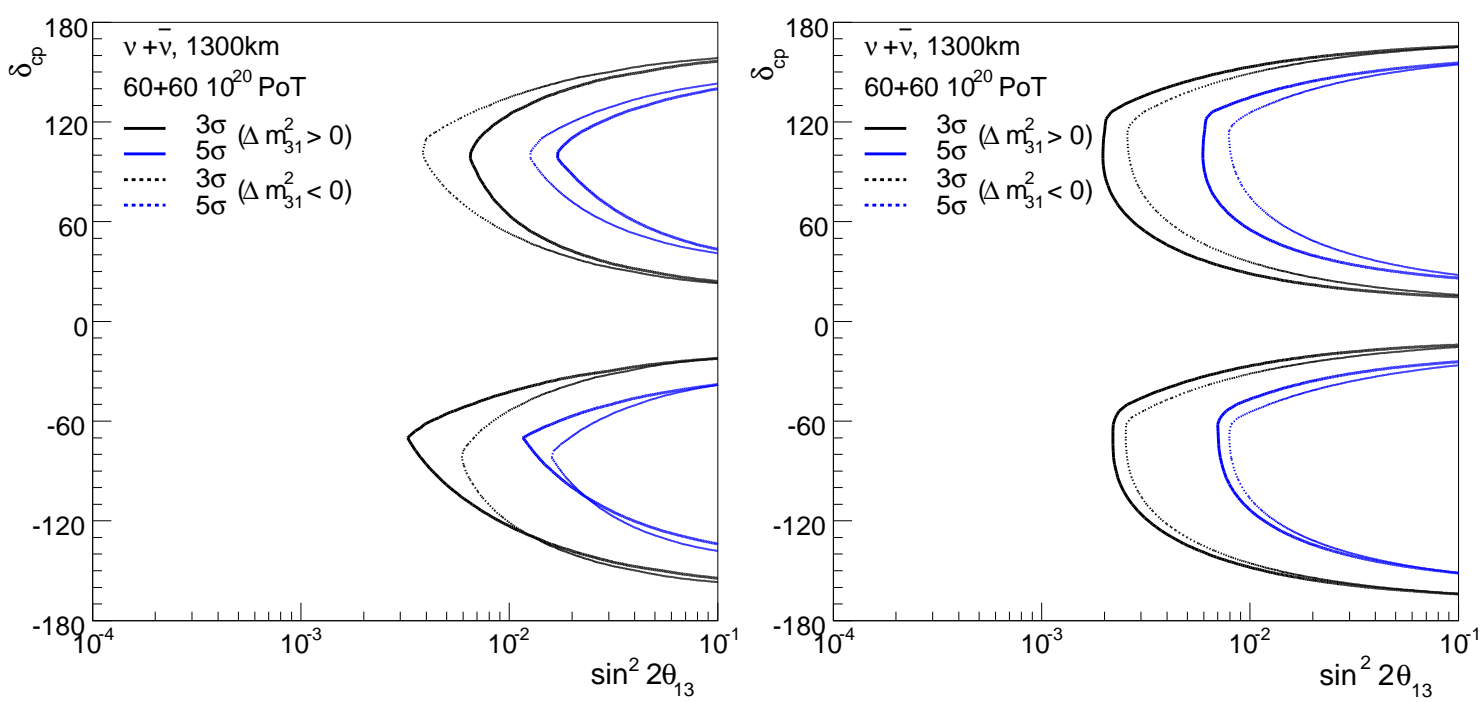

FIG. 16: 3 sigma and 5 sigma confidence level exclusion limits for determining $C P$ violation in $\sin ^{2} 2 \theta_{13}$ versus $\delta_{C P}$ for statistical and systematic errors (left hand plot). This is for a $300 \mathrm{kT}$ water Cherenkov detector with a total exposure of $120 \times 10^{20}$ POT. The right hand side is for statistical errors alone. The solid (dashed) lines are for normal (reversed) mass ordering. We assume $10 \%$ systematic errors on the background for this plot.

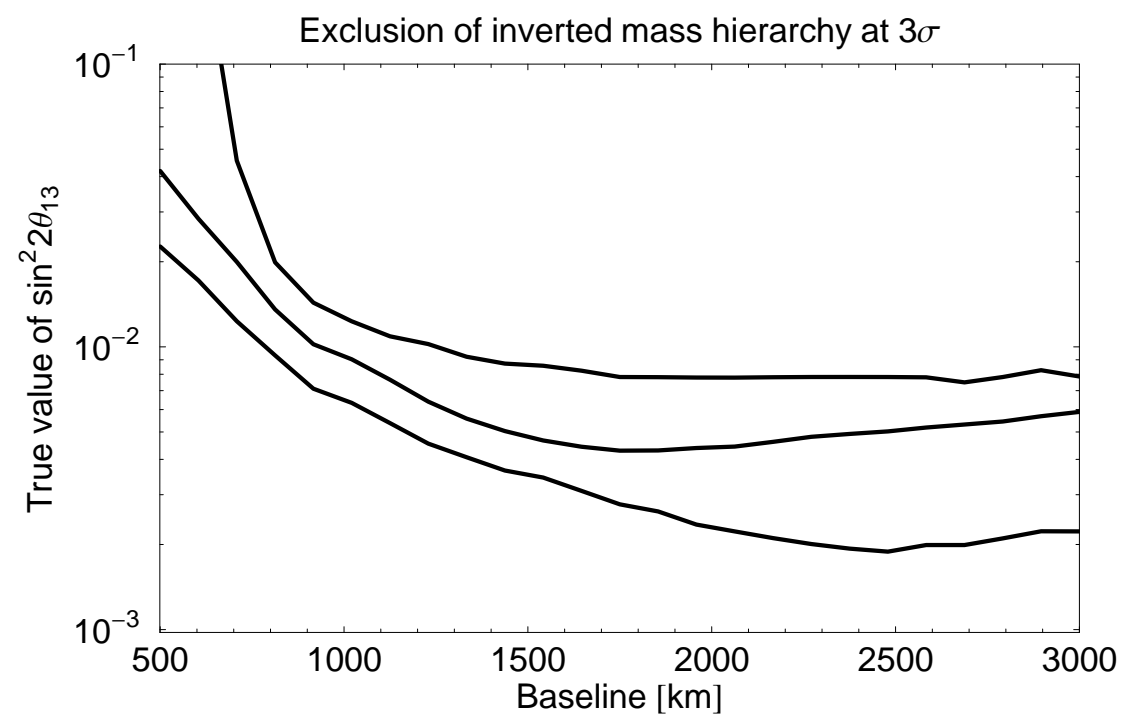

FIG. 17: Discovery reach for a normal mass hierarchy at $3 \sigma$ for CP fractions 0 (lower-most line, best case), 0.5 (middle line) and 1 (uppermost line, worst case) as a function of the baseline. The detector mass, beam power and exposure are kept the same for all baselines. For further explanation of the plot please see [67]. 


\subsubsection{Liquid Argon Detector}

If a 100 kTon fiducial mass liquid argon time projection chamber can be built at one of the DUSEL sites, it could be used as a long baseline neutrino oscillation detector. We have assumed that such a detector can have $80 \%$ efficiency for all charged current electron neutrino events and has background rejection capability as described in Section 10.2. This virtually rejects all NC and $\mathrm{CC}$ backgrounds. We further assume that the detector will have resolution characterized by $20 \% / \sqrt{E / G e V}$ for non-quasielastic events and 5\% $/ \sqrt{E / G e V}$ for quasielastic events. The spectra that result from these assumptions is displayed in Figure 18 for the same parameters and exposure as Figure 9.

The parameter resolutions and sensitivity limits for the $100 \mathrm{kT}$ liquid Argon TPC at DUSEL are shown in Figures 19 to 22. If there is no excess of electron events observed then we can set a limit on the value of $\sin ^{2} 2 \theta_{13}$ as a function of $\delta_{C P}$. Such sensitivity limits are shown in Figure 20. The range of parameters over which the mass hierarchy can be resolved is shown in Figure 21. We have chosen to display the limits separately for the two mass hierarchies. The region to the right hand side of each curve excludes the opposite mass hierarchy at the respective confidence level. Similarly the range of parameters over which CP violation can be established (i.e. determine that $\delta_{C P}$ is not 0 or $\pi$ ) is displayed in Figure 22. The comments we made regarding dependence on exposure, baseline, and oscillation parameters for the water Cherenkov detector are equally applicable to the sensitivities one would obtain with the liquid argon detector placed at DUSEL. 

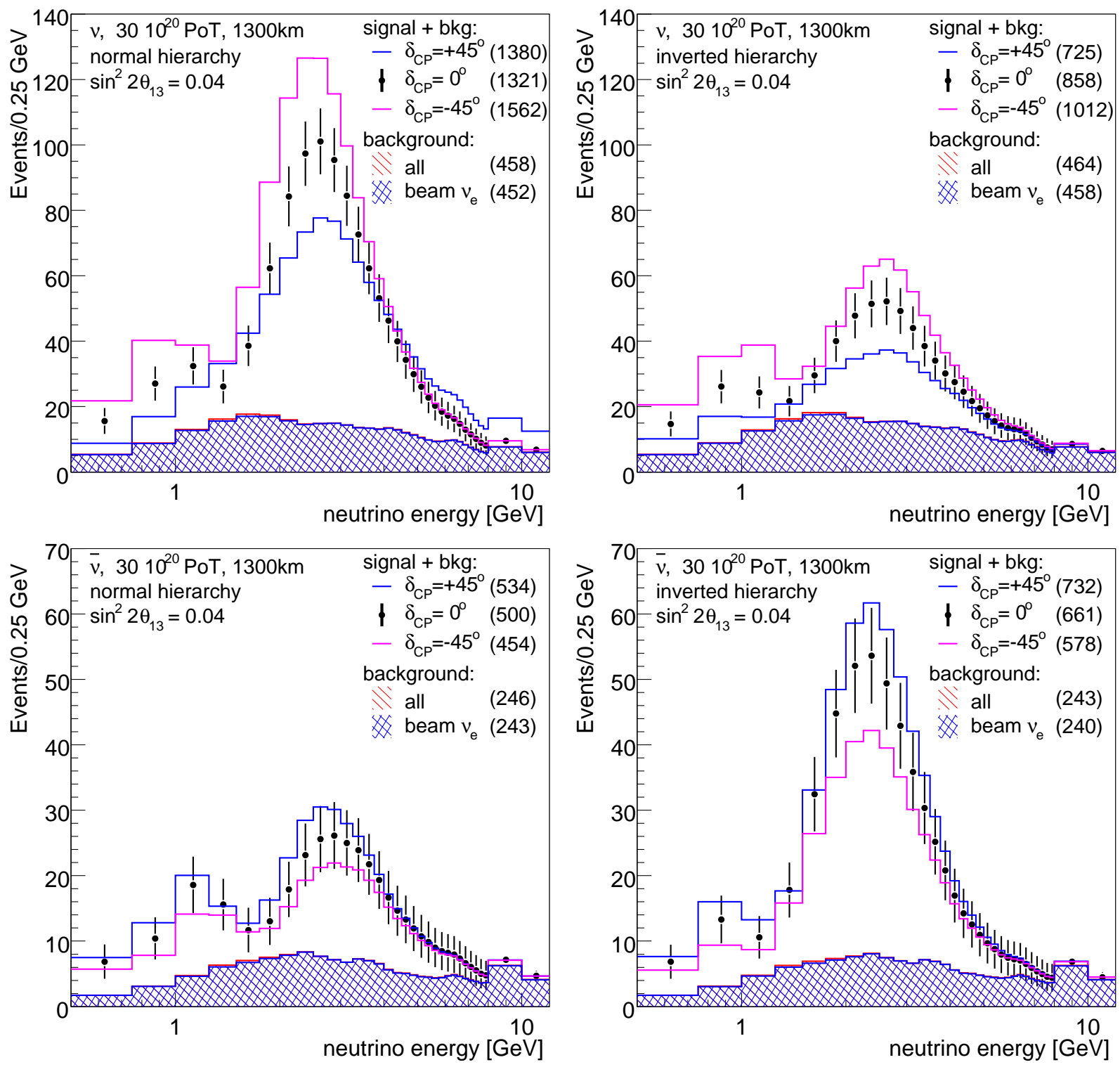

FIG. 18: Simulation of detected electron neutrino (top plots) and anti-neutrino (bottom plots) spectrum (left for normal hierarchy, right for reversed hierarchy) for 3 values of the CP parameter $\delta_{C P},-45^{\circ}, 0^{\circ}$, and $-45^{\circ}$, including background contamination. This simulation is for $100 \mathrm{kT}$ of LAr detector (with the performance described in the text) placed at DUSEL, $1300 \mathrm{~km}$ away from FNAL. The hatched histogram shows the total background, which is dominated by the $v_{e}$ beam background. The other parameters and running conditions are shown in the figure. 

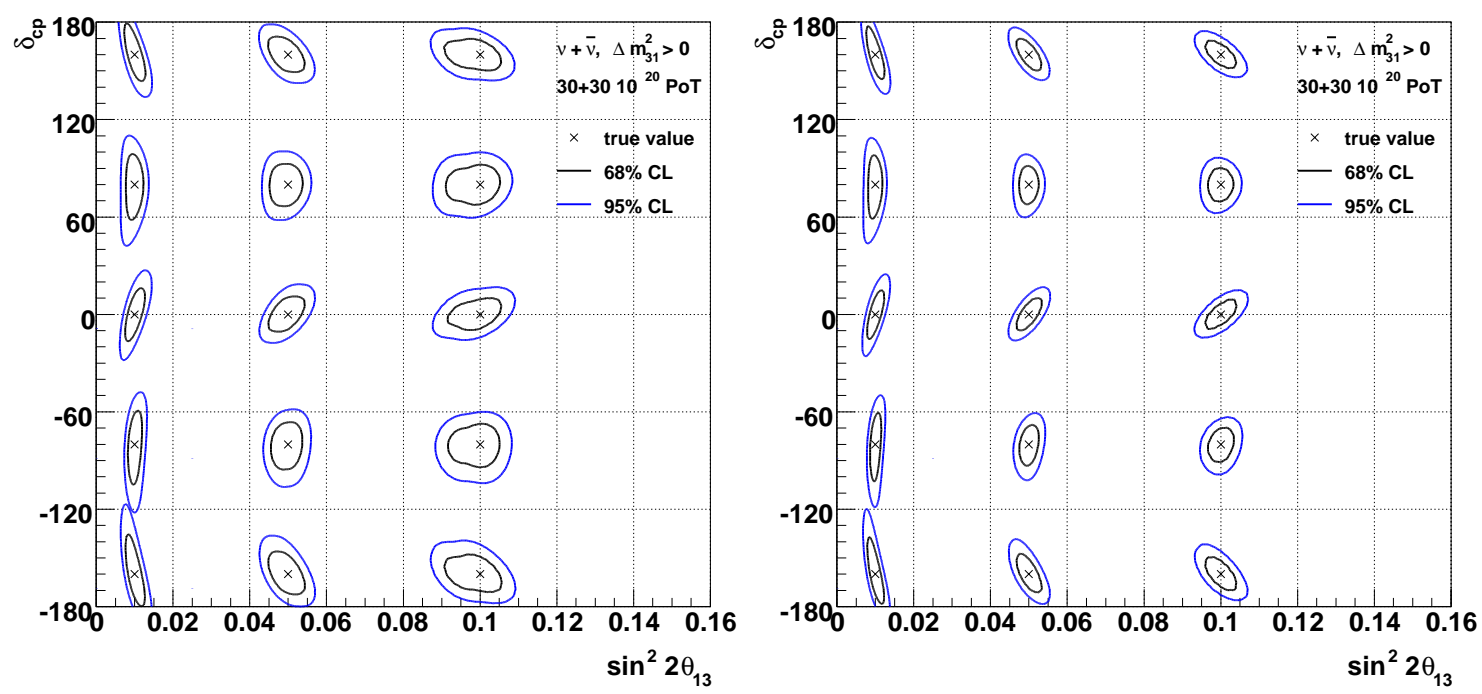

FIG. 19: $90 \%$ and $95 \%$ confidence level error contours in $\sin ^{2} 2 \theta_{13}$ versus $\delta_{C P}$ for statistical and systematic errors (left hand plot) for 15 test points. This plot is for a 100 kTon liquid Argon TPC placed at DUSEL $1300 \mathrm{~km}$ away from FNAL. This is for combining both neutrino and anti-neutrino data. The right hand side is for statistical errors alone. This plot was made for normal mass hierarchy. We assume $10 \%$ systematic errors on the background for this plot.
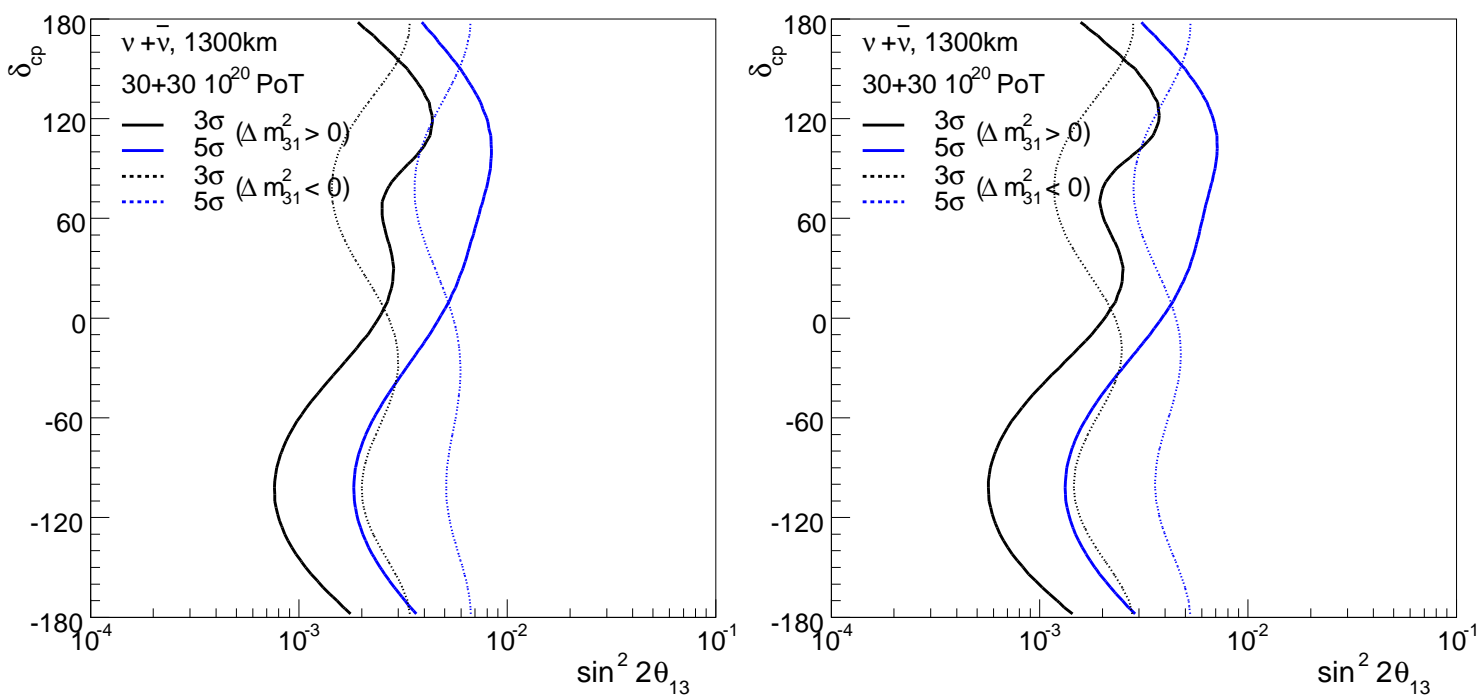

FIG. 20: 3 sigma and 5 sigma confidence level exclusion limits for determining a non-zero value for $\theta_{1} 3$ in $\sin ^{2} 2 \theta_{13}$ versus $\delta_{C P}$ for statistical and systematic errors (left hand plot). This plot is for a 100 kTon liquid Argon TPC placed at DUSEL $1300 \mathrm{~km}$ away from FNAL. This is for combining both neutrino and antineutrino data. The right hand side is for statistical errors alone. The solid (dashed) lines are for normal (reversed) mass ordering. We assume 10\% systematic errors on the background for this plot. 

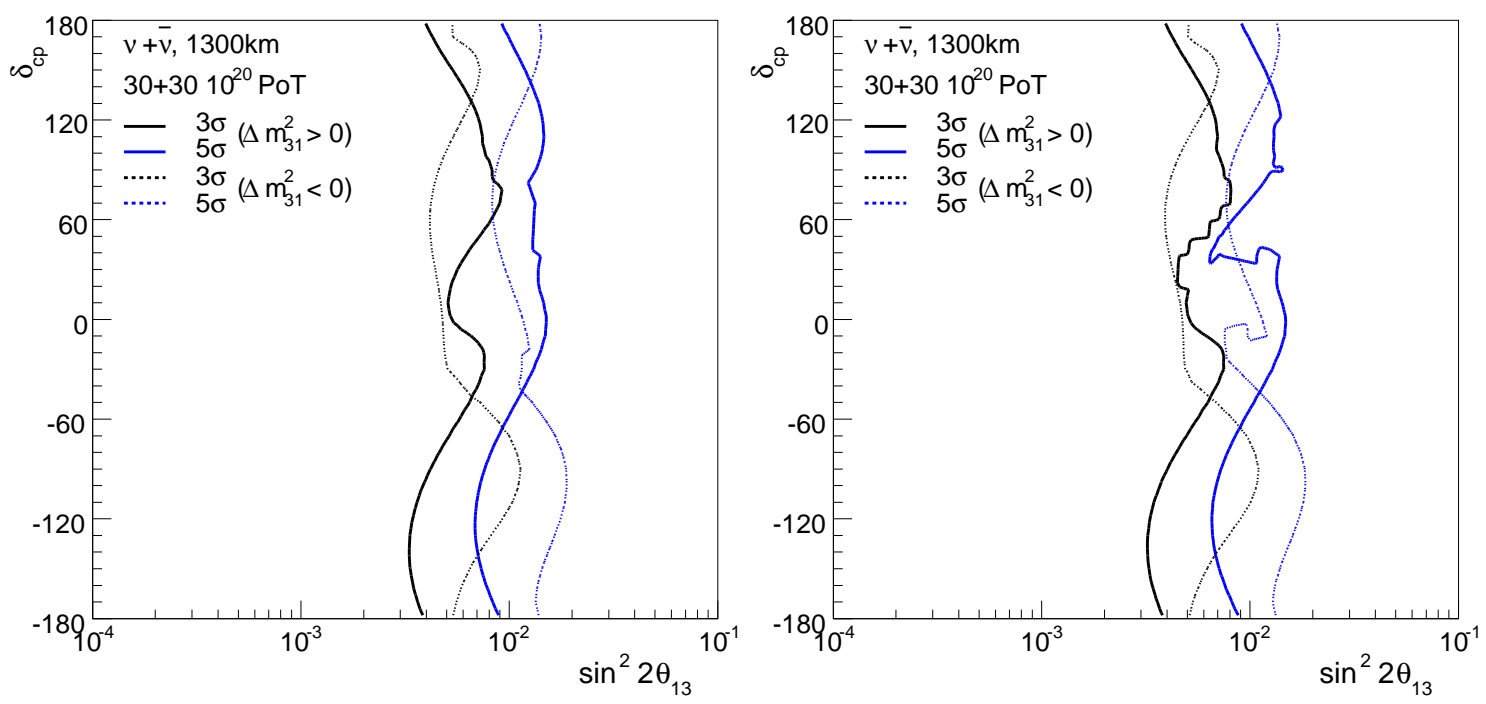

FIG. 21: 3 sigma and 5 sigma confidence level exclusion limits for determining the mass hierarchy in $\sin ^{2} 2 \theta_{13}$ versus $\delta_{C P}$ for statistical and systematic errors (left hand plot). This plot is for a $100 \mathrm{kTon}$ liquid Argon TPC placed at DUSEL $1300 \mathrm{~km}$ away from FNAL. This is for combining both neutrino and antineutrino data. The right hand side is for statistical errors alone. The solid (dashed) lines are for normal (reversed) mass ordering. We assume 10\% systematic errors on the background for this plot.
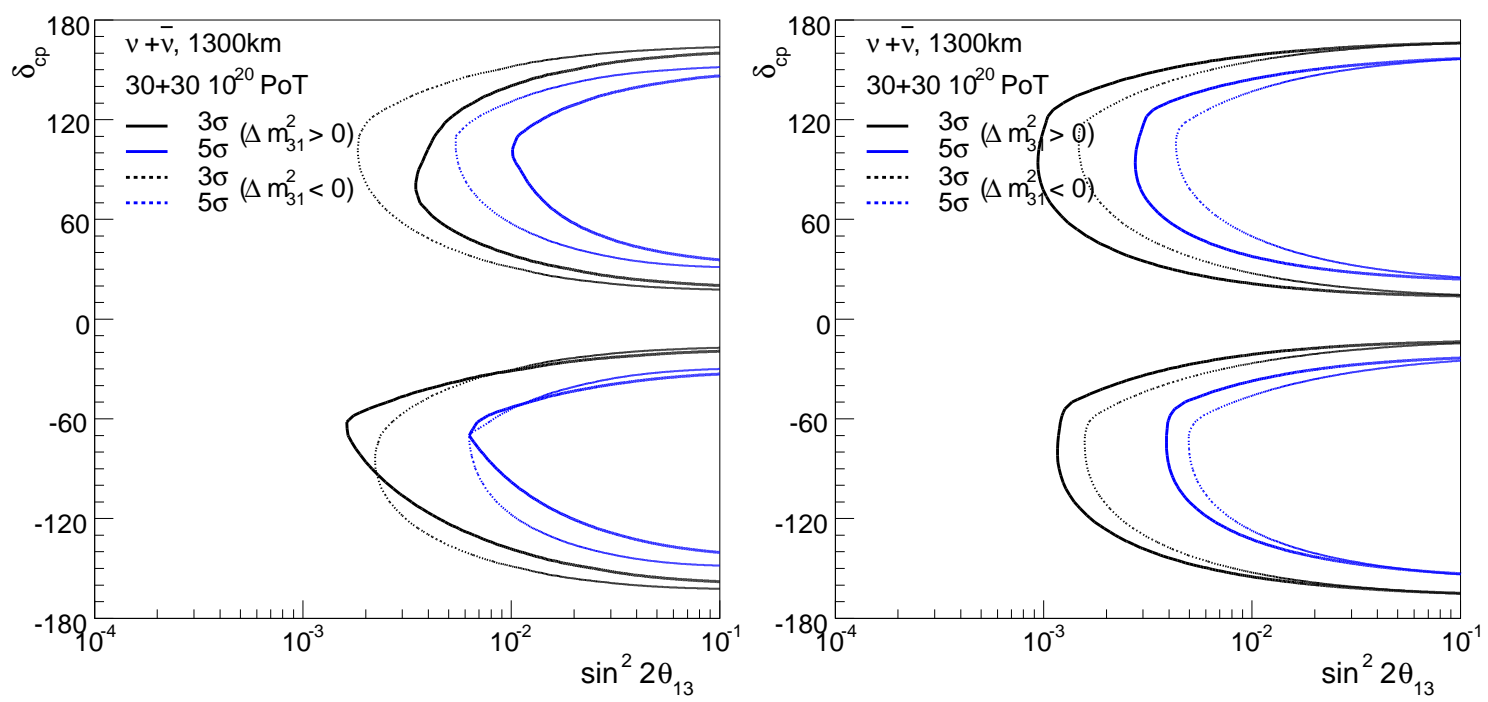

FIG. 22: 3 sigma and 5 sigma confidence level exclusion limits for determining $C P$ violation in $\sin ^{2} 2 \theta_{13}$ versus $\delta_{C P}$ for statistical and systematic errors (left hand plot). This plot is for a 100 kTon liquid Argon TPC placed at DUSEL $1300 \mathrm{~km}$ away from FNAL. This is for combining both neutrino and anti-neutrino data. The right hand side is for statistical errors alone. The solid (dashed) lines are for normal (reversed) mass ordering. We assume $10 \%$ systematic errors on the background for this plot. 


\subsection{Sensitivity of a NuMI based off axis program}

In this section we present the sensitivity results for several of the scenarios using NuMI off-axis neutrinos which we studied. The following assumptions were made for this part of the study. We have attempted to make the calculations as directly comparable to the previous section as possible. But there are small differences. These are also listed below.

- The source of the proton beam used to create neutrinos will be the Fermilab Main Injector.

- Current planning at Fermilab to maximize the proton intensity from the Main Injector via a series of staged upgrades, will in fact occur over the next decade.

- The possibility of a new source, such as the HINS (High Intensity Neutrino Source[17]), or alternative ideas to improve the Main Injector, is considered as the final stage of the upgrade path, ultimately providing an annual proton intensity of $2 \times 10^{21}$ protons per year.

- Though the Fermilab Main Injector can be operated with extracted proton energies of less than $120 \mathrm{GeV}$, we have assumed that the optimum operation is at $120 \mathrm{GeV}$, based on total delivered beam power.

- The neutrino beam which we are considering is the existing NuMI beam, which is a conventional horn focusing beam, capable of producing both neutrino and anti-neutrino beams (by reversing the current in the horns). We do not consider any reconfiguration or modification to the existing 2-meter diameter, 675-meter long decay pipe.

- We assume that upgrades to targets, horns, shielding and cooling systems will be required to accommodate proton intensities significantly higher than that for which the facility was designed.

- We do assume that the target and horn configuration can be adjusted to optimize neutrino rates.

- We assume that detectors situated in NuMI off-axis locations will most likely be sited on or near the surface. We do not discus the detector designs required to reject the backgrounds from cosmic ray interactions associated with a surface location.

- We have used an efficiency of $80 \%$ and a neutral current rejection factor of 0.001 , consistent with the parameters of a Liquid Argon detector.

- We assume that the background is known with a systematic error of 5\%. 
- The assumption on oscillation parameters is stated at the beginning of this section. For the calculation here these parameters as well as the matter density are assumed to be fixed.

- In our first pass analysis our sensitivities have been generated assuming no prior knowledge of either $\sin ^{2} 2 \theta_{13}$, the mass hierarchy or $\delta_{C P}$. Iteration of the sensitivity calculation for the mass hierarchy and $\delta_{C P}$ have also been done such that for values of $\sin ^{2} 2 \theta_{13} \geq 0.02$, the angle is known (as will be the case from Phase I experiments). For values $\leq 0.02$ we assume the angle is unknown.

As a cross-check and starting point for our study, we have calculated the sensitivities for a 20 kton NOvA detector (see Figure 23). Our results are consistent with those produced for the NOvA project Technical Design Report. Note, for this and all subsequent figures, the dashed line is placed at the current Chooz limit.

For each scenario we assumed that the Phase II program consisted of running for an equal time in neutrino and anti-neutrino mode. We show the plots for an integrated proton intensity of $30 \times 10^{20}$ in each mode. We also assume that the NOvA detector continues to take data during Phase II. The new detectors are all Liquid Argon technology.

The fi rst scenario considered was placing a $100 \mathrm{kton}$ detector at the 1 st maximum, i.e. simply increasing the mass and efficiency of the NOvA configuration. The sensitivities are shown in Figure 24.

The second scenario we studied, was to place a $100 \mathrm{kton}$ detector at a baseline of $700 \mathrm{~km}$ and an off-axis angle of $57 \mathrm{mrad}(40 \mathrm{~km})$. This location corresponds to the second oscillation maximum, where the matter effects are small (due to the lower energy of the neutrinos), but the CP effects are large. The NOvA detector is the only detector at the first maximum site $(\mathrm{L}=810,14 \mathrm{mrad}$ off axis). These results are shown in Figure 25. We find that with this configuration, running neutrinos and anti-neutrinos, the sensitivity to the mass hierarchy flattens over the range of possible $\delta_{C P}$ values, but the discovery potential is limited to values of $\theta_{13}$ relatively close to the current limit.

A third scenario was to split the mass between the two locations, 50 ktons at each the first and second maximum. These results are in Figure 26.

Finally, a fourth scenario was the same as the third except that the detectors at each site were 100 ktons.

We summarize our studies in Table VIII. Because some of the scenarios studied have the benefit of "flattening" the sensitivity over $\delta_{C P}$, we have included the sensitivity limits for both $50 \%$ and $100 \%$ coverage of the $\delta_{C P}$ space. We have also included in this table the limits which can be reached as the Phase I program evolves, and as the Liquid Argon technology also evolves.

From these studies we conclude the following: 
- $\sin ^{2} 2 \theta_{13}$ down to 0.02 can be measured by the Phase I (NOvA) experiments. Phase I experiments however, have limited or no sensitivity to determining the mass hierarchy, and essentially no sensitivity to $\delta_{C P}$.

- If $\sin ^{2} 2 \theta_{13}$ is large, i.e. $>0.04$ a Phase II experiment using the NuMI beam can be designed specifically to determine the mass hierarchy. Such an experiment is like our 4th scenario (see Table VIII), with two massive detectors placed at the first and second maximum sites. In this experiment the mass hierarchy can be resolved for all values of $\delta_{C P}$. The experiment also has sensitivity to $\delta_{C P}$.

- The most interesting and complex situation to plan for, is if Phase I experiments indicate that $0.02<\sin ^{2} 2 \theta_{13}<0.04$. In this case we find that the configurations studied for the NuMI Off-Axis option can have relatively good sensitivity to determine the mass hierarchy, as well as some sensitivity to $C P$.

- If Phase I experiments conclude that $\sin ^{2} 2 \theta_{13}<0.02$ the Phase II program can continue the search. Continued running, more protons and larger more efficient detectors, placed at the 1st maximum (the NOvA site), allows one to reach sensitivities to well below $10^{-2}$, (of the order $\sim 0.003$ ) as can be seen in our first scenario.

\subsection{Comparison of sensitivity estimates}

A summary of the sensitivity reach for non-zero $\theta_{13}, \mathrm{CP}$ violation and the sign of $\Delta m_{31}^{2}$ for 6 different combinations of beams, baselines, detector technologies, and exposure is presented in Table IX. Several more configurations for the off-axis scenario are presented in Table VIII. The sensitivity reach is given as the lowest $\sin ^{2} 2 \theta_{13}$ value at which at least $50 \%$ of $\delta_{c p}$ values will have $\geq 3 \sigma$ reach. For this table we use the mass hierarchy with the worst sensitivity to determine the minimal value of $\sin ^{2} 2 \theta_{13}$ for which $\geq 50 \%$ of $\delta_{c p}$ values will have $\geq 3 \sigma$ sensitivity to a particular measurement. We estimated these values of $\sin ^{2} 2 \theta_{13}$ from the studies and plots discussed in Sections 13.1 and 13.2. We note that different options are sensitive to different values of $\delta_{c p}$, such that being sensitive to $50 \% \delta_{c p}$ values does not necessarily imply that a given experimental option is sensitive to the same region of oscillation parameter phase space as another.

We compare the wide-band FNAL to DUSEL program, option (4), with the narrow-band offaxis NuMI-based program, option (2), for the same exposure of $6.8 \mathrm{MW} . y r$ (1 experimental year is defined as $1.7 \times 10^{7}$ seconds). This is equivalent to an integrated exposure of $60 \times 10^{20}$ protons-ontarget for proton beam energies of $120 \mathrm{GeV}$. We assume equal amounts of exposure for neutrinos and anti-neutrino (reverse horn current) running. A liquid Argon TPC with a total mass of 100 
$\mathrm{kT}$ is assumed as the detector technology of choice for the purpose of the comparison. We note that slightly different assumptions on the systematic uncertainties on the oscillation parameters and backgrounds went into the sensitivity estimates for NuMI off-axis (5\% uncertainty on the background) and the wide-band FNAL to DUSEL options (10\% uncertainty on the background). The effect of the different assumptions is $\leq 15 \%$ variation on the value of $\sin ^{2} 2 \theta_{13}$ at which the sensitivity reaches $50 \%$ of $\delta_{C P}$. We find that for the same exposure of $6.8 \mathrm{MW} . \mathrm{yr}$, and the same liquid Argon TPC detector technology, the wide-band FNAL to DUSEL approach has significantly better sensitivity to CP violation, the sign of $\Delta m_{31}^{2}$, and comparable sensitivity to non-zero values of $\theta_{13}$. To illustrate the improvement in sensitivity over the existing program, the sensitivities of the current NOvA experiment (as shown in Figure 23) at the same exposure, are summarized as option (1) in Table IX [85].

The value of $\sin ^{2} 2 \theta_{13}$ at which at least $50 \%$ of $\delta_{c p}$ values will have $\geq 3 \sigma$ reach as a function of exposure for the NuMI ME beam at $810 \mathrm{~km}$ (labeled NOvA*), and the wide-band $120 \mathrm{GeV}$ beam at $1300 \mathrm{~km}$ (labeled WBB-120 s) is summarized in Figure 28 from reference [68]. A LAr TPC is the detector technology assumed for NOvA* and WBB- $120_{\mathrm{s}}$. We find that after reaching an exposure of 2 MT.MW. $10^{7}$ seconds (for 100kT LAr and a $120 \mathrm{GeV}$ beam, this is an exposure of $10^{22}$ protons-on-target), the mass hierarchy- $\delta_{c p}$ degeneracy is sufficiently resolved for the NOvA* approach (option (2)) - and the sensitivity to CP violation approaches that of the wide-band beam at the $1300 \mathrm{~km}$ baseline. For the mass hierarchy, the wide-band FNAL to DUSEL approach always has significantly better sensitivity independent of the exposure. Option (3) in Table IX is a NuMIbased program with a $50 \mathrm{kT}$ detector at the 1st oscillation maximum running concurrently with another 50kT module placed at the 2nd oscillation maxima. We find that option (3) has worse sensitivity to non-zero values of $\theta_{13}$ when compared to option (2) and slightly better sensitivity to the sign of $\Delta m_{31}^{2}$.

Option (5) summarizes the FNAL to DUSEL sensitivity when the 100 kT LAr TPC of option (4) is replaced by a $300 \mathrm{kT}$ water Cherenkov detector. We find that the sensitivity worsens due to the lower signal statistics and higher NC backgrounds in a water Cherenkov detector. We can recover some of the lost sensitivity by doubling the exposure of the water Cherenkov detector as shown in option (6). For the same exposure, the FNAL to DUSEL program with a $300 \mathrm{kT}$ water Cherenkov detector, option (5), has the same sensitivity to $\mathrm{CP}$ violation as the NuMI based program with a 100 kT LAr TPC in options (2) and (3) and significantly better sensitivity to the sign of $\Delta m_{31}^{2}$. We find the FNAL to DUSEL program with a $300 \mathrm{kT}$ water Cherenkov detector has similar sensitivity to non-zero $\theta_{13}$ as the NuMI based program with two $50 \mathrm{kT}$ LAr TPC's at the 1st and 2nd oscillation maxima, option (3).

We summarize the comparison studies as follows: 
- $\sin ^{2} 2 \theta_{13}$ down to 0.02 can be measured by the Phase I (NOvA) experiments. Phase I experiments however, have limited or no sensitivity to determining the mass hierarchy, and essentially no sensitivity to $\delta_{C P}$.

- All Phase II experimental options will improve the sensitivity to CP violation by at least an order of magnitude over the existing Phase I program.

- Given the same exposure and detector technology (LAr TPC), the FNAL to DUSEL program with a wide band beam has significantly better overall sensitivity to neutrino oscillations when compared to a shorter baseline NuMI based program with an off-axis beam.

- The FNAL to DUSEL program with a $300 \mathrm{kT}$ water Cherenkov detector has similar sensitivity to CP violation when compared to a NuMI off-axis program with a $100 \mathrm{kT}$ LAr TPC, and significantly better sensitivity to the sign of $\Delta m_{31}^{2}$.

- A NuMI off-axis program with two $50 \mathrm{kT}$ LAr TPCs at the 1st and 2nd oscillation maxima at baselines of 810 and $700 \mathrm{~km}$ respectively has marginally better sensitivity to the sign of $\Delta m_{31}^{2}$ but significantly worse sensitivity to non-zero $\theta_{13}$ when compared with putting the full $100 \mathrm{kT}$ mass at the 1 st oscillation maxima. 

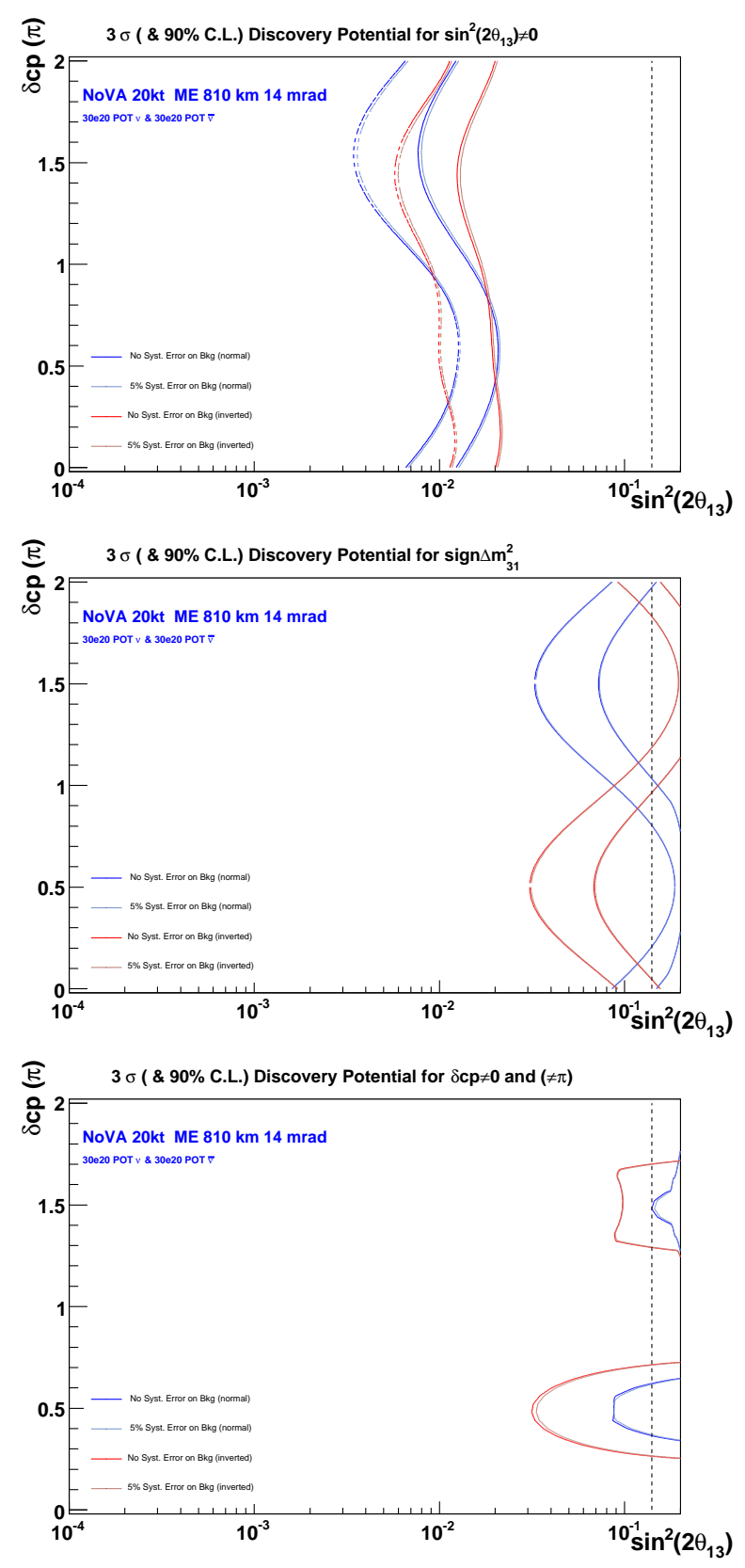

FIG. 23: 90\%, 3 sigma confidence level exclusion limits for determining a non-zero value for $\theta_{1} 3$ (top), for excluding the opposite mass hierarchy (middle), and for excluding CP violation (bottom) in $\sin ^{2} 2 \theta_{13}$ versus $\delta_{C P}$. These plots (blue for normal and red for reversed hierarchy) are for a 20 kTon NOvA detector placed at the off-axis location on the NuMI beam-line with a total exposure of $60 \times 10^{20}$ protons, and for combining both neutrino and anti-neutrino data. 5\% background systematic errors are assumed. 

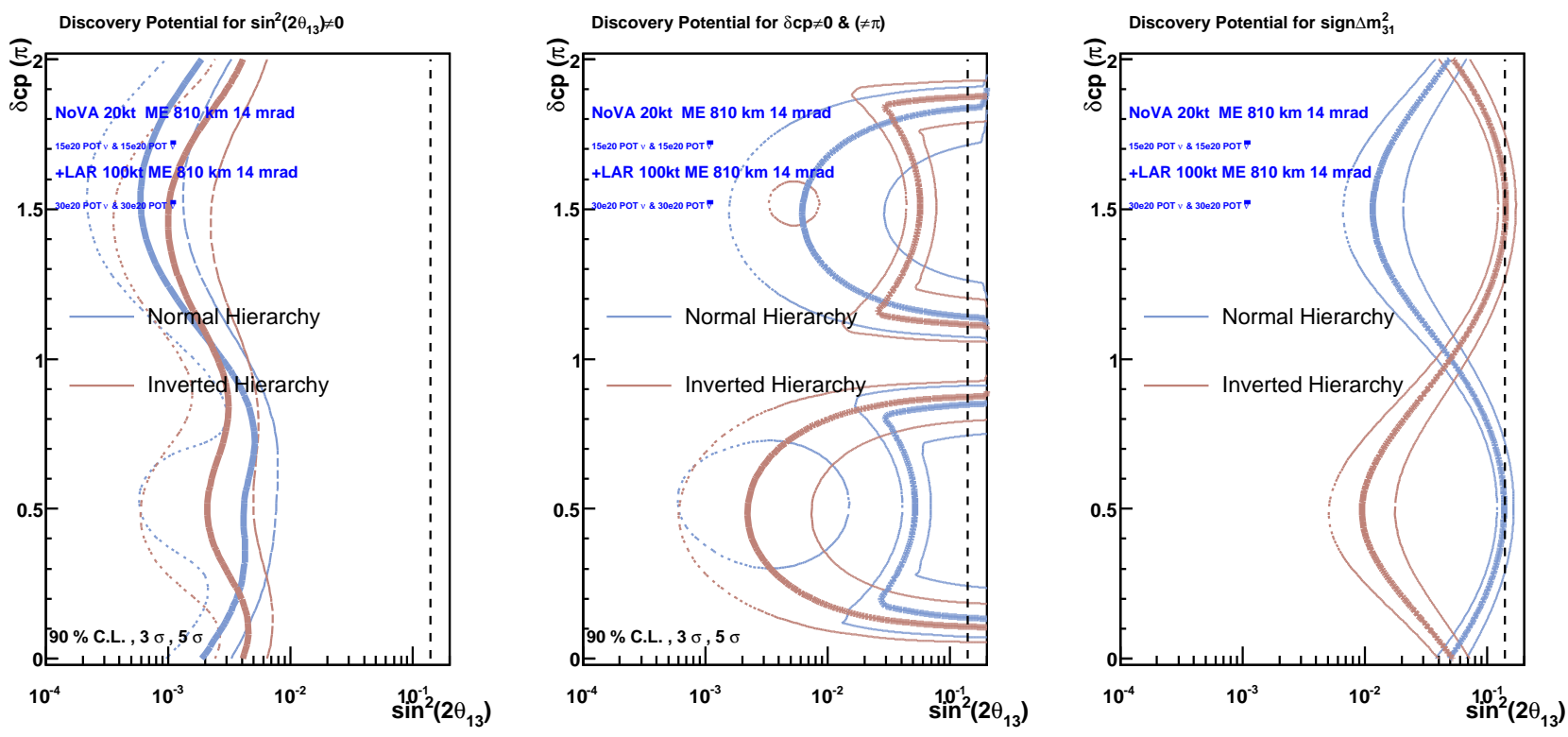

FIG. 24: Scenario $1: 90 \%, 3 \sigma$, and $5 \sigma$ confidence level exclusion limits for determining a non-zero value of $\theta_{13}$ (left), for excluding $\mathrm{CP}$ violation (center), and for excluding the opposite mass hierarchy (right), in $\sin ^{2} 2 \theta_{13}$ versus $\delta_{C P}$.
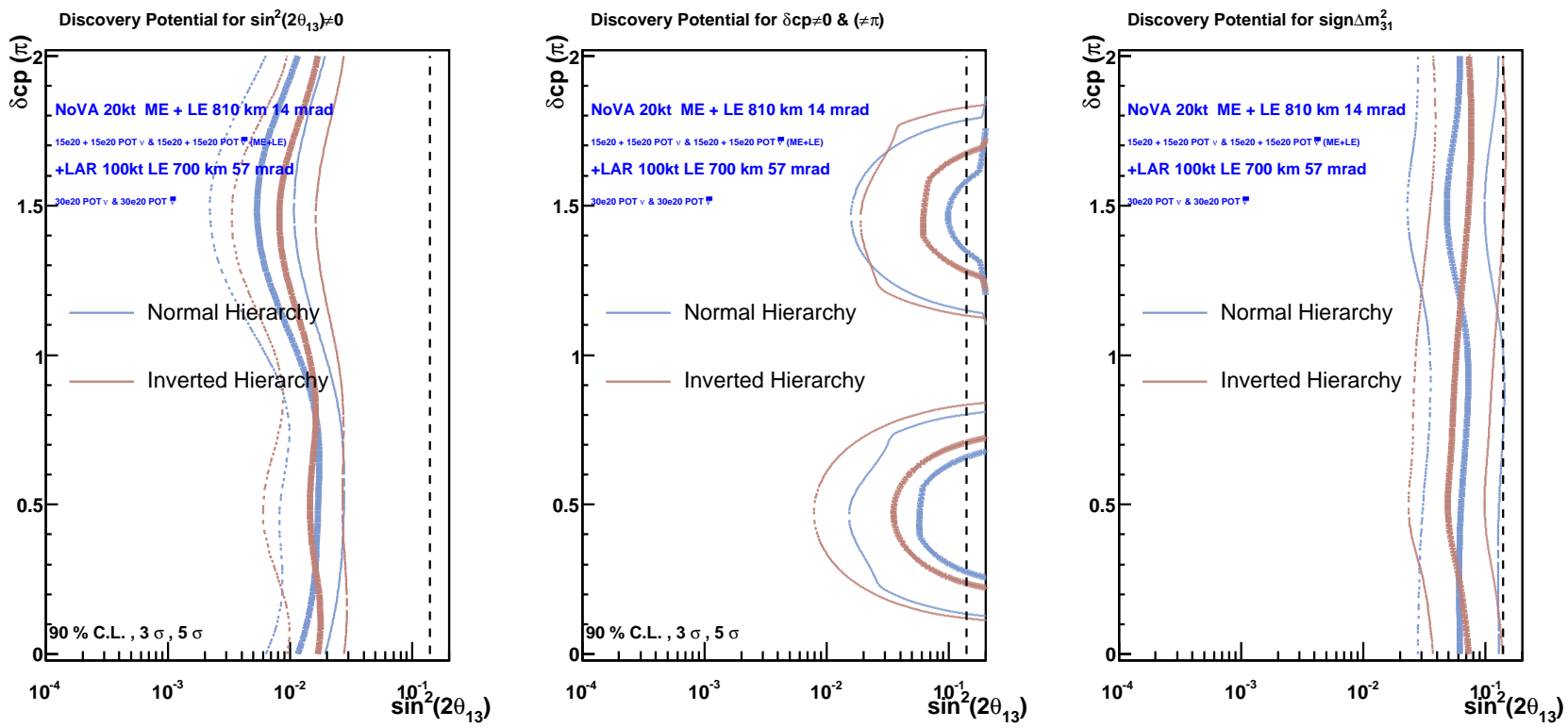

FIG. 25: Scenario $2: 90 \%, 3 \sigma$, and $5 \sigma$ confidence level exclusion limits for determining a non-zero value of $\theta_{13}$ (left), for excluding $\mathrm{CP}$ violation (center), and for excluding the opposite mass hierarchy (right), in $\sin ^{2} 2 \theta_{13}$ versus $\delta_{C P}$. 

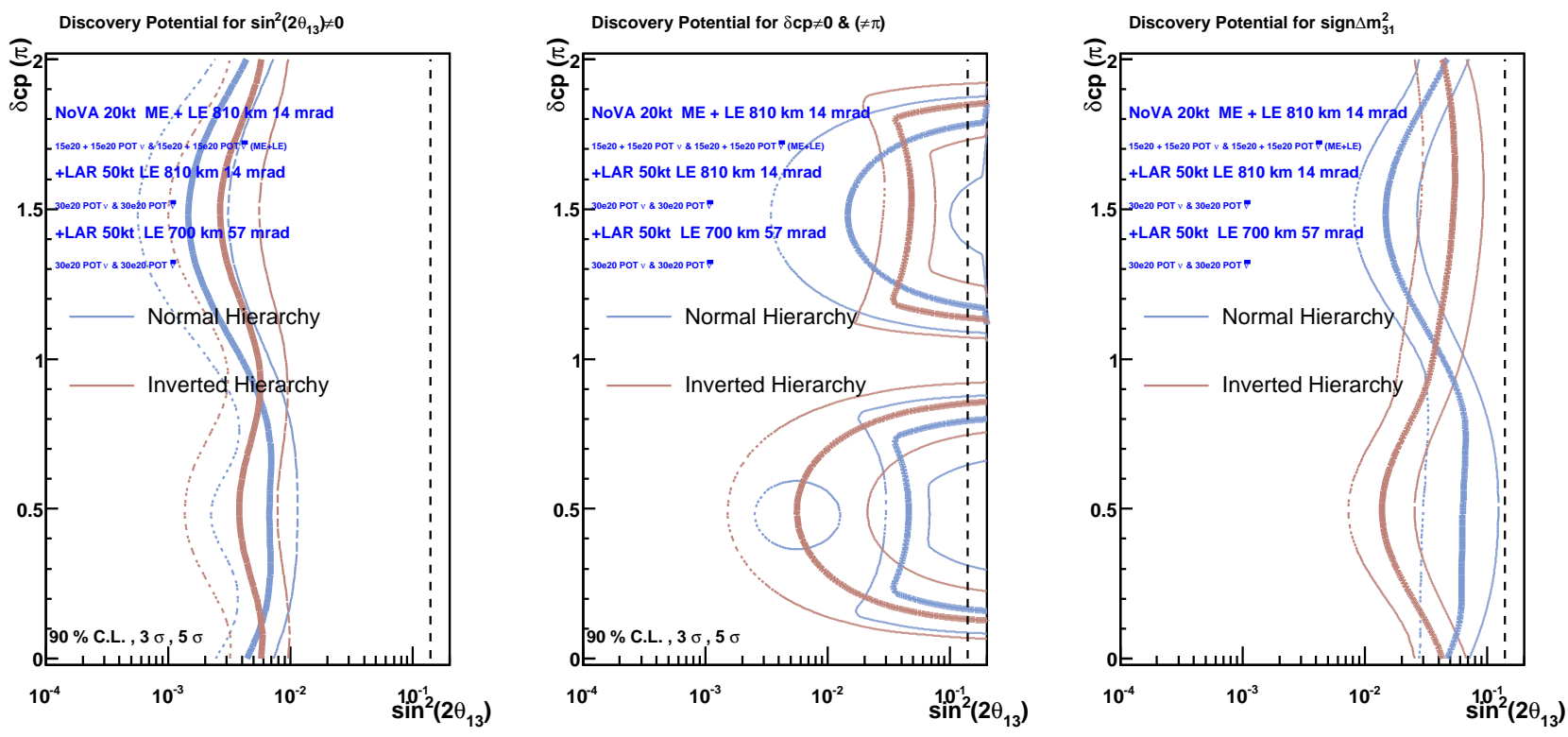

FIG. 26: Scenario $3: 90 \%, 3 \sigma$, and $5 \sigma$ confidence level exclusion limits for determining a non-zero value of $\theta_{13}$ (left), for excluding $\mathrm{CP}$ violation (center), and for excluding the opposite mass hierarchy (right), in $\sin ^{2} 2 \theta_{13}$ versus $\delta_{C P}$.
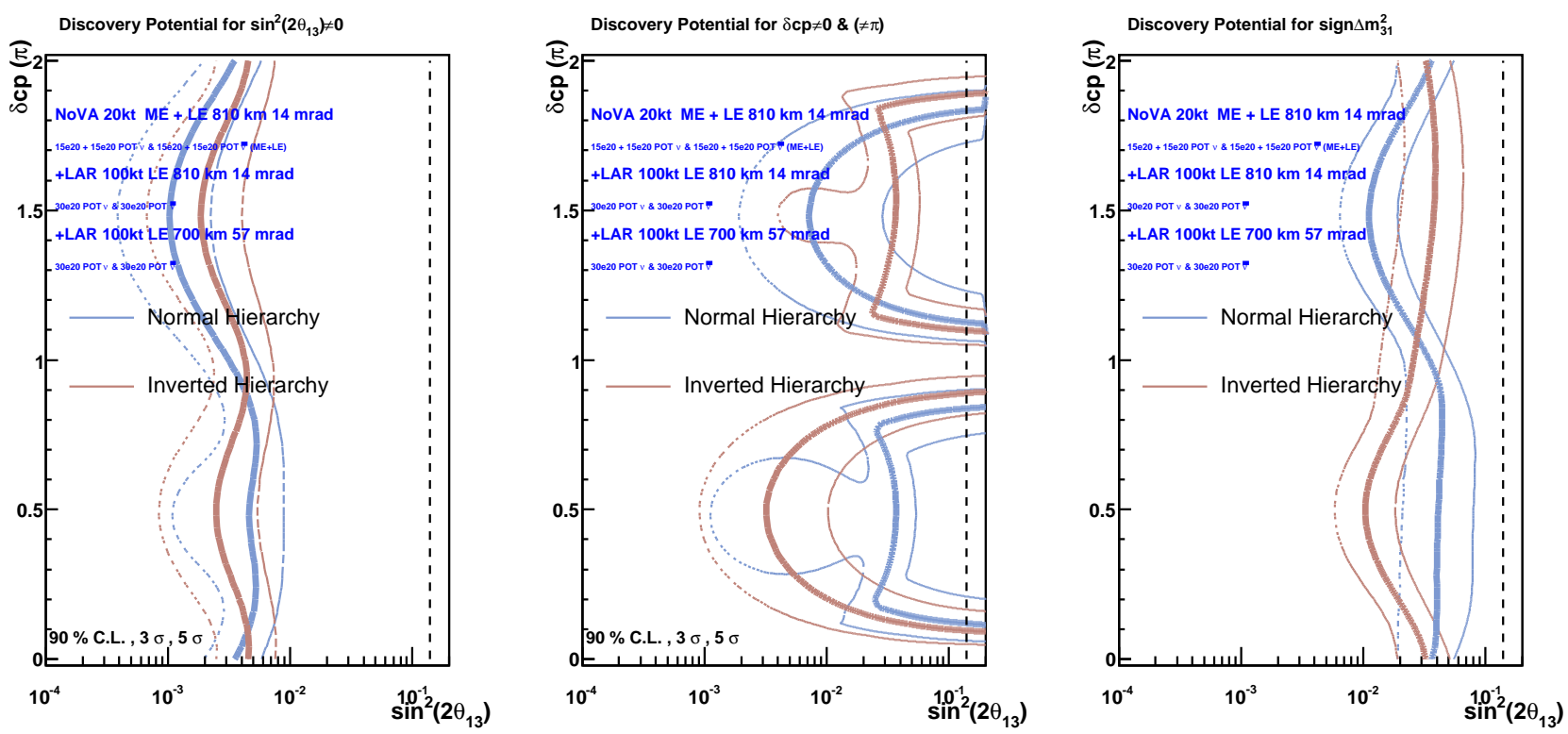

FIG. 27: Scenario $4: 90 \%, 3 \sigma$, and $5 \sigma$ confidence level exclusion limits for determining a non-zero value of $\theta_{13}$ (left), for excluding CP violation (center), and for excluding the opposite mass hierarchy (right), in $\sin ^{2} 2 \theta_{13}$ versus $\delta_{C P}$. 


\begin{tabular}{|c|c|c|c|c|c|}
\hline $\begin{array}{c}\text { Detector Location } \\
(\mathrm{L}(\mathrm{km}), \theta(\mathrm{mr})) \\
\text { (beam tune })\end{array}$ & $\begin{array}{c}\text { Detector } \\
\text { (technology/mass) }\end{array}$ & $\begin{array}{l}\text { Exposure } \\
\text { POT }(v) \\
/ \operatorname{POT}(\bar{v})\end{array}$ & $\sin ^{2} 2 \theta_{13}$ & $\operatorname{sgn}\left(\Delta m_{31}^{2}\right)$ & $\mathrm{CPV}$ \\
\hline $810,14(\mathrm{ME})$ & $\mathrm{NO} v \mathrm{~A} / 20 \mathrm{kt}$ & $15 /-$ & $0.018 / 0.030$ & $0.17 / \mathrm{NA}$ & NA \\
\hline $810,14(\mathrm{ME})$ & $\mathrm{NO} v \mathrm{~A} / 20 \mathrm{kt}$ & $15 / 15$ & $0.018 / 0.024$ & $0.16 / \mathrm{NA}$ & NA \\
\hline $810,14(\mathrm{ME})$ & $\mathrm{NO} v \mathrm{~A} / 20 \mathrm{kt}$ & $30 / 30$ & $0.012 / 0.020$ & $0.10 / \mathrm{NA}$ & NA \\
\hline $810,14(\mathrm{ME})$ & $\mathrm{NO} v \mathrm{~A} / 20 \mathrm{kt}$ & $30 / 30$ & $0.007 / 0.013$ & $0.08 / 0.20$ & NA \\
\hline $810,14(\mathrm{ME})$ & $+\mathrm{LAr} / 5 \mathrm{kt}$ & $30 / 30$ & & & \\
\hline $810,14(\mathrm{ME})$ & $\mathrm{NO} v \mathrm{~A} / 20 \mathrm{kt}$ & $30 / 30$ & $0.004 / 0.009$ & $0.05 / 0.15$ & $0.07 / \mathrm{NA}$ \\
\hline $810,14(\mathrm{ME})$ & $+\mathrm{LAr} / 20 \mathrm{kt}$ & $30 / 30$ & & & \\
\hline Scenario 1 & & & & & \\
\hline $810,14(\mathrm{ME})$ & $\mathrm{NO} v \mathrm{~A} / 20 \mathrm{kt}$ & $30 / 30$ & $0.0018 / 0.005$ & $0.03 / 0.12$ & $0.03 / \mathrm{NA}$ \\
\hline $810,14(\mathrm{ME})$ & + LAr/100kt & $30 / 30$ & & & \\
\hline Scenario 2 & & & & & \\
\hline 810, 14(ME/LE) & $\mathrm{NOvA} / 20 \mathrm{kt}$ & $30 / 30$ & $0.011 / 0.018$ & $0.05 / 0.07$ & $0.07 / \mathrm{NA}$ \\
\hline 700,57 (LE) & $+\mathrm{LAr} / 100 \mathrm{kt}$ & $30 / 30$ & & & \\
\hline Scenario 3 & & & & & \\
\hline $810,14(\mathrm{ME} / \mathrm{LE})$ & NOvA $/ 20 \mathrm{kt}$ & $30 / 30$ & & & \\
\hline $810,14(\mathrm{LE})$ & $+\mathrm{LAr} / 50 \mathrm{kt}$ & $30 / 30$ & $0.0035 / 0.006$ & $0.033 / 0.06$ & $0.035 / \mathrm{NA}$ \\
\hline $700,57(\mathrm{LE})$ & $+\mathrm{LAr} / 50 \mathrm{kt}$ & $30 / 30$ & & & \\
\hline Scenario 4 & & & & & \\
\hline $810,14(\mathrm{ME} / \mathrm{LE})$ & NOvA / $20 \mathrm{kt}$ & $30 / 30$ & & & \\
\hline $810,14(\mathrm{LE})$ & $+\mathrm{LAr} / 100 \mathrm{kt}$ & $30 / 30$ & $0.0027 / 0.0046$ & $0.030 / 0.042$ & $0.022 / \mathrm{NA}$ \\
\hline 700,57 (LE) & + LAr/100kt & $30 / 30$ & & & \\
\hline
\end{tabular}

TABLE VIII: Sensitivity comparisons for all NuMI Off-axis scenarios that were evaluated. These numbers were calculated with the normal hierarchy assumption. The first three cases represent three possible stages of the Phase I (NOvA ) program. The values given represent the value of $\sin ^{2} 2 \theta_{13}$ where a $3 \sigma$ determination of the parameter can be made for $50 \%(/ 100 \%)$ of the possible values of $\delta_{C P}$. Note that for determining the sensitivity to mass hierarchy and $\delta_{C P}$, for values of $\sin ^{2} 2 \theta_{13}>0.02$ we assume that the angle is known (i.e. from Phase I experiments.) 


\begin{tabular}{|c|l|c|c|c|c|c|c|}
\hline Option & Beam & Baseline & Detector & Exposure (MW.yr $)$ & $\theta_{13} \neq 0$ & CPV & $\operatorname{sgn}\left(\Delta m_{31}^{2}\right)$ \\
\hline \hline$(1)$ & NuMI ME, 0.9 & $810 \mathrm{~km}$ & NOvA 20 kT & 6.8 & 0.015 & $>0.2$ & 0.15 \\
\hline$(2)$ & NuMI ME, 0.9 & $810 \mathrm{~km}$ & LAr $100 \mathrm{kT}$ & 6.8 & 0.002 & 0.03 & 0.05 \\
$(3)$ & NuMI LE, $0.9^{\circ}, 3.3^{\circ}$, & $810,700 \mathrm{~km}$ & LAr 2 $\times 50 \mathrm{kT}$ & 6.8 & 0.005 & 0.04 & 0.04 \\
$(4)$ & WBLE $120 \mathrm{GeV}, 0.5^{\circ}$ & $1300 \mathrm{~km}$ & LAr 100 kT & 6.8 & 0.0025 & 0.005 & 0.006 \\
\hline (5) & WBLE $120 \mathrm{GeV}, 0.5^{\circ}$ & $1300 \mathrm{~km}$ & WCe 300 kT & 6.8 & 0.006 & 0.03 & 0.011 \\
$(6)$ & WBLE $120 \mathrm{GeV}, 0.5^{\circ}$ & $1300 \mathrm{~km}$ & WCe 300 kT & 13.6 & 0.004 & 0.012 & 0.008 \\
\hline
\end{tabular}

TABLE IX: Comparison of the sensitivity reach of different long baseline experiments. The sensitivity is given as the value of $\sin ^{2} 2 \theta_{13}$ at which $50 \%$ of $\delta_{c p}$ values will have $\geq 3 \sigma$ reach for the choice of mass hierarchy with worst sensitivity. We assume equal amounts of $v$ and $\bar{v}$ running in the total exposure. The assumption on running time is $1.7 \times 10^{7}$ seconds of running per year. Also see Table VIII. 


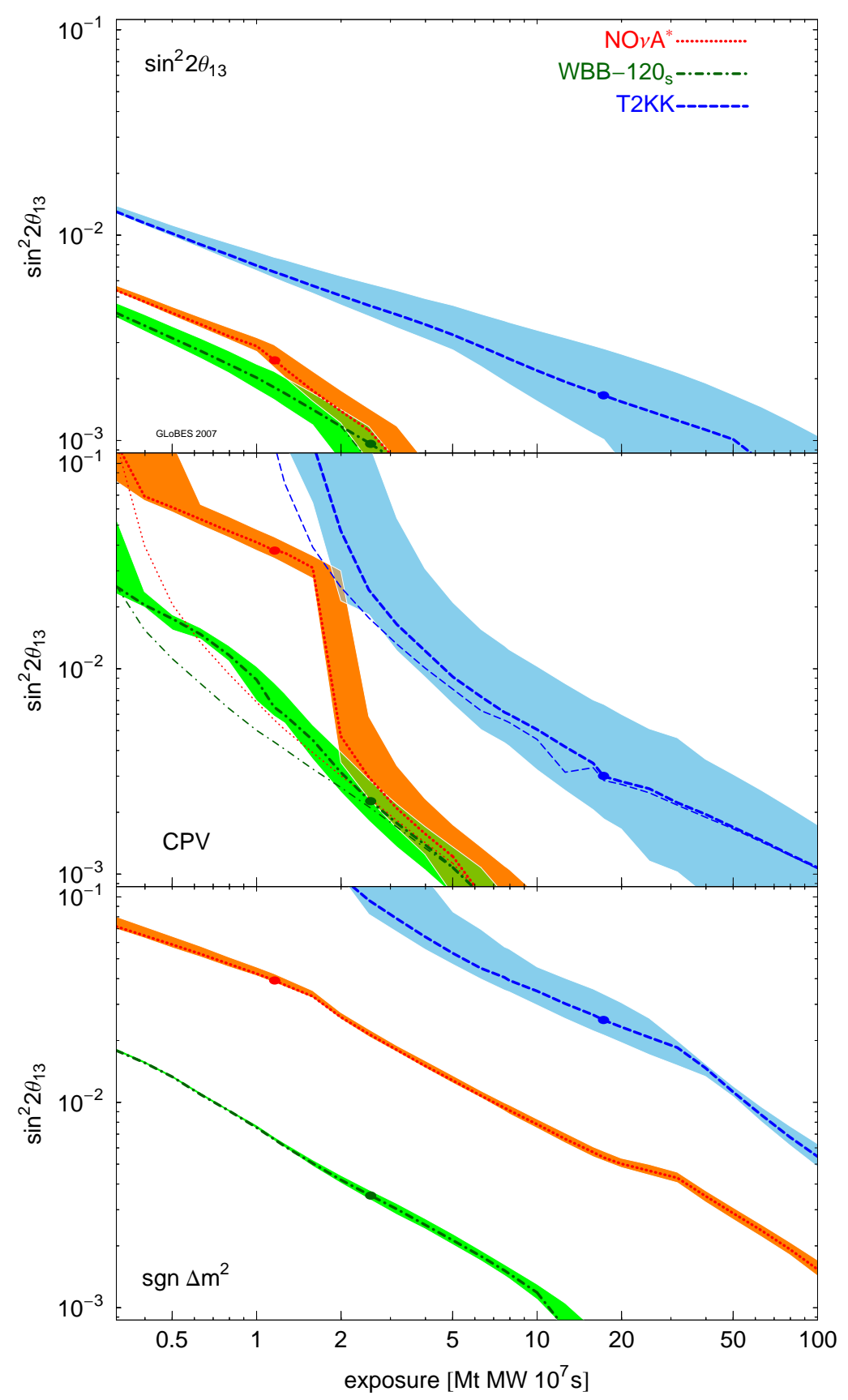

FIG. 28: The $\sin ^{2} 2 \theta_{13}$ reach at $3 \sigma$ for the discovery of nonzero $\sin ^{2} 2 \theta_{13}$ (top), CP violation(middle), and the normal hierarchy (bottom) as a function of exposure. The curves are for a fraction of $\delta_{C P}$ of 0.5 , which means that the performance will be better for $50 \%$ of all values of $\delta_{C P}$, and worse for the other $50 \%$. The light curves in the CPV panel are made under the assumption that the mass hierarchy is known to be normal. The shaded regions result by varying the systematic uncertainties from $2 \%$ (lower edge) to $10 \%$ (upper edge). This figure is reproduced from [68]. 


\section{SENSITIVITY TO NON-ACCELERATOR PHYSICS}

A well instrumented very large detector, in addition to its accelerator based neutrino program, could be sensitive to nucleon decay which is one of the top priorities in fundamental science. All of the detector technologies we consider will lead to enhanced detection and study of neutrinos from natural sources such as the Sun, Earth's atmosphere and lithosphere, and past and current supernova explosions. To achieve these goals, the key issues are cosmogenic backgrounds and low energy thresholds $(\sim 5 \mathrm{MeV})$; the first primarily depends on the depth of the detector and the second depends on the depth, the radioactivity from the materials used in the detector and in the surrounding rock, and the detector noise (photosensor noise in the case of a water Cherenkov detector, and electronic noise in the case of a liquid argon TPC).

In this section we briefly summarize the potential of a large detector for nucleon decay and astrophysical sources of neutrinos. We also comment on the technical requirements on the detector. For each topic we attempt to identify where the requirements of the accelerator program match and where they diverge.

\subsection{Improved Search for Nucleon Decay}

Theoretical Motivation: While current experiments show that the proton lifetime exceeds about $10^{33}$ years, its ultimate stability has been questioned since the early 1970's in the context of theoretical attempts to arrive at a unified picture of the fundamental particles - the quarks and leptons - and of their three forces: the strong, electromagnetic and weak. These attempts of unification, commonly referred to as "Grand Unification", have turned out to be supported empirically by the dramatic meeting of the strengths of the three forces that is found to occur at high energies in the context of so-called "Supersymmetry", as well as by the magnitude of neutrino masses that is suggested by the discovery of atmospheric and solar neutrino oscillations. One of the most crucial and generic predictions of grand unification, however, is that the proton must ultimately decay into leptonic matter such as a positron and a meson, revealing quark-lepton unity. A class of wellmotivated theories of grand unification, based on the symmetry of $\mathrm{SO}(10)$ and Supersymmetry, which have the virtue that they successfully describe the masses and mixings of all quarks and leptons including neutrinos, and which also explain the origin of the excess of matter over anti-matter through a process called "leptogenesis", provide a conservative (theoretical) upper limit on the proton lifetime which is within a factor of ten of the current lower limit. This makes the discovery potential for proton decay in a next-generation experiment high.

From a broader viewpoint, proton decay, if found, would provide us with a unique window to view physics at truly short distances - less than $10^{-30} \mathrm{~cm}$., corresponding to energies greater than 
$10^{16} \mathrm{GeV}$ - a feature that cannot be achieved by any other means. It would provide the missing link of Grand Unification. Last, but not least, it would help ascertain our ideas about the origin of an excess of matter over anti-matter that is crucial to the origin of life itself. In this sense, and given that the predictions of a well-motivated class of Grand Unified theories for proton lifetime are not far above the current limit, the need for an improved search for proton decay through a next-generation detector seems compelling. The theoretical guidance provided by some promising models points towards the need for improved searches for proton decaying into $\bar{v} K^{+}$and $e^{+} \pi^{0}$ modes with lifetimes less than about $2 \times 10^{34}$ and $10^{35}$ years, respectively. Should proton decay be discovered in these modes, valuable insight would be gained by searches for other related modes including $\mu^{+} \pi^{0}$ and $\mu^{+} K^{0}$.

Current status of experimentation: The "classical" proton decay mode, $p \rightarrow e^{+} \pi^{0}$, can be efficiently detected with low background. At present, the best limit on this mode $\left(>5.4 \times 10^{33}\right.$ yr, 90\% CL) comes from a 92 kTon-yr exposure of Super-Kamiokande. The detection efficiency of $44 \%$ dominated by final-state $\pi^{0}$ absorption or charge-exchange in the nucleus, and the expected background is 2.2 events/Mton-yr. The mode $p \rightarrow \bar{v} K^{+}$, is experimentally more difficult in water Cherenkov detectors due to the unobservable neutrino and the fact that the kaon is below Cherenkov threshold. The present limit from Super-Kamiokande is the result of combining several channels, the most sensitive of which is $K^{+} \rightarrow \mu^{+} v$ accompanied by a de-excitation signature from the remnant ${ }^{15} N$ nucleus. Monte Carlo studies suggest that this mode should remain background free for the foreseeable future. The present limit on this mode is $>2.2 \times 10^{33} \mathrm{yr}(90 \% \mathrm{CL})$.

Requirements for the next stage of experimentation: Since the lifetime of the nucleon is unknown, and could range from just above present limits to many orders of magnitude greater, increases in sensitivity by factors of a few are insufficient to motivate new experiments. Thus, continued progress in the search for nucleon decay inevitably requires much larger detectors than Super-Kamiokande. The efficiency for detection of the $e^{+} \pi^{0}$ mode is dominated by pion absorption effects in the nucleus, and cannot be improved significantly. An order of magnitude improvement in this mode can only be achieved by running Super-Kamiokande for an additional 30-40 more years, or by constructing an order of magnitude larger experiment. The decay modes of the nucleon are also unknown, and produce quite different experimental signatures, so future detectors must be sensitive to most or all of the kinematically allowed channels. Moreover, the enormous mass and exposure required to improve significantly on existing limits (and the unknowable prospects for positive detection) underline the importance of any future experiment's ability to address other important physics questions while waiting for the proton to decay.

New facilities under consideration: A variety of technologies for discovery of nucleon decay have been discussed. Of these, underground water Cherenkov appears to be the only one capable of reaching lifetimes of $10^{35}$ years or greater. Cooperative, parallel studies of a future underground 
water Cherenkov proton decay experiment are underway in the U.S. and Japan. The proposed designs range from $300 \mathrm{kTon}$ (14 times Super-Kamiokande) to 1 Mton. Liquid Argon or scintillation techniques have also been discussed in the proton decay community and may have significant efficiency advantages for certain modes that are dominant in a certain broad class of SUSY theories. Liquid Argon time projection chambers potentially offer very detailed measurements of particle physics events with superb resolution and particle identification. Liquid Argon feasibility will be demonstrated in the near future with the operation of a 600-ton ICARUS detector. If expectations are correct, it should have a sensitivity that is equivalent to a 6000-ton water Cherenkov detector in the $p \rightarrow \bar{v} K^{+}$mode. The liquid scintillator approach is presently being explored with the 1 kTon KamLAND experiment. It should also have enhanced sensitivity to this mode by directly observing the $K^{+}$by $d E / d x$ and observing the subsequent $K^{+} \rightarrow \mu^{+} v$ decay.

Performance and feasibility: Detailed Monte Carlo studies, including full reconstruction of simulated data, indicate that the water detectors could reach the goal of an order of magnitude improvement on anticipated nucleon decay limits from Super-Kamiokande. With sufficient exposure, clear discovery of nucleon decay into $e^{+} \pi^{0}$ would be possible even at lifetimes of (few) $\times 10^{35}$ years where present analyses would be background-limited, by tightening the selection criteria. For instance, with a detection efficiency of $18 \%$, the expected background is only 0.15 events/Mton-yr, ensuring a signal:noise of $4: 1$ even for a proton lifetime of $10^{35}$ years. A water Cherenkov detector would also provide a decisive test of super-symmetric $\mathrm{SO}(10)$ grand unified theory by reaching a sensitivity of a (few) $\times 10^{34}$ years for the $\bar{v} K^{+}$mode.

As we have discussed, a much smaller liquid argon could do particularly well on the mode $\bar{v} K^{+}$ as the efficiency could be as much as 10 times larger than that in the water Cherenkov detectors due to the extraordinary bubble chamber-like pattern recognition capabilities. Due to this, a single observed event could be powerful evidence for a discovery. The $e^{+} \pi^{0}$ mode however would be limited by the smaller size of these detectors.

The search for n-nbar oscillation is another test of baryon non-conservation. While this is not one of the favorite predictions of conventional SUSY grand unification, this process, taking place

in the nuclear potential, can reach an equivalent sensitivity to baryon non-conservation of $10^{35}$ years.

\subsection{Observation of Natural Sources of Neutrinos}

All of the detector technologies we consider will lead to enhanced detection and study of neutrinos from natural sources such as the Sun, Earth's atmosphere and lithosphere, and past and current supernova explosions. There may also be previously unsuspected, natural neutrino sources that appear when the detector mass reaches the hundreds of kilotons scale. The liquid scintillator tech- 
nique is of particular note here because it could allow the detection of low energy antineutrinos from Earth's lithosphere. This physics, however, requires low energy thresholds which are difficult to obtain without eliminating cosmogenic background by locating the detector at great depth and with careful selection of materials with low intrinsic radioactivity for the detector construction. The low activity concerns become important if we attempt to push the threshold to below $\sim 5 \mathrm{MeV}$. The low activity requirement is not essential for accelerator physics.

Solar neutrinos have already been observed in the Super-Kamiokande and SNO detectors. If the large detector concepts discussed here result in construction of the underground experiment, it may become possible to increase the observable event rate enough to clearly observe spectral distortion in the $<5$ to $14 \mathrm{MeV}$ region. One could also measure the as yet undetected hep solar neutrinos (with an endpoint of $18.8 \mathrm{MeV}$ ) well beyond the ${ }^{8} \mathrm{~B}$ endpoint ( $14 \mathrm{MeV}$ ). These measurements would require a very comprehensive understanding of the detector systematics and energy resolution, but a better determination of the solar spectrum as well as detection of the day-night effect with high statistics would represent a significant advance in the evolution of solar nuclear physics measurements.

The observation of supernova neutrino events in a large neutrino detector of the type being discussed in this report is straightforward and has historical precedent. The SN 1987A supernova, in fact, was seen by two large water Cherenkov detectors (11 events in Kamiokande-II (total mass $3 \mathrm{kT}$ ) and 8 events in IMB (total mass $7 \mathrm{kT}$ )) that were active in proton decay searches at that time. The predicted occurrence rate for neutrino- observable supernovas (from our own galaxy and of order $10 \mathrm{kpc}$ distant) is about 1 per 20 years, so events will be very rare. However, the information from a single event, incorporating measured energies and time sequence for tens of thousands of neutrino interactions, obtained by a very large neutrino detector, could provide significantly more information than has ever been obtained before about the time evolution of a supernova. In addition to obtaining information about supernova processes, the small numbers of SN1987a neutrino events have been extensively used to limit fundamental neutrino properties. Supernova processes continue to have very high interest because of the recent detection of the acceleration of the rate of expansion of the universe using type Ia supernova. Recent work has shown that diffuse neutrino events from past core collapse supernova (which produce neutrino bursts) could be used to gain independent knowledge on the cosmological evolution parameters[? ]. Therefore detection of supernova neutrinos, either as a burst from a single supernova or as a diffuse source from past supernovas, should be a key mission of the multipurpose detector facility.

Recently, there has been substantial progress in the detection of relic supernova neutrinos using the inverse beta decay reaction $\bar{v}_{e}+p \rightarrow e^{+}+n$. This ability could be obtained with some futher investment as described below. Neutrons are presently invisible in water Cherenkov detectors. After thermalizing, they are captured by free protons in the water, emitting a $2.2 \mathrm{MeV}$ gamma which 
is well below a typical threshold and which is also overwhelmed by the large radon backgrounds at such energies. However, if we were to dissolve gadolinium in the form of gadolinium (tri)chloride, $\mathrm{GdCl}_{3}$, in the water (the price of gadolinium has dropped three orders of magnitude in recent years, making such a detector affordable) then the experiment would become sensitive to the neutron capture gamma cascade (total energy $=8.0 \mathrm{MeV}$ ) produced by $\mathrm{Gd}$ following positron emission from the inverse beta reaction [64]. With a concentration of $0.1 \% \mathrm{Gd}\left(0.2 \% \mathrm{GdCl}_{3}\right)$ by mass, over $90 \%$ of the neutrons will be visibly captured on Gd rather than on protons.

By requiring coincident signals, i.e., a positron's Cherenkov light followed shortly thereafter $(<100 \mu s)$ and very close to the same spot by the gamma cascade of a captured neutron, backgrounds to the diffuse supernova neutrino signal could be greatly reduced. Diffuse supernova neutrino background (DSNB) models vary, but with the Gd in the water the 50kton Super-Kamiokande should see about five DSNB events each year above $10 \mathrm{MeV}$ with essentially no background. One can easily imagine a next-generation water Cherenkov detector seeing $>100$ supernova relic neutrinos every year. Adding gadolinium would greatly improve the response to a supernova within our own galaxy as well, allowing the deconvolution of the various neutrino signals (charged current, neutral current, elastic scattering) and, among other things, doubling the pointing accuracy back to the progenitor star. Such a detector would also be sensitive to late black hole formation to much longer times than at present, since the distinctive coincident inverse beta signals can be distinguished from the usual singles backgrounds. An abrupt cutoff of these coincident signals would be the unmistakable signature of a singularity being born.

The continued study of atmospheric in the large underground detector will provide useful additions to the program carried out so successfully by the Super-Kamiokande Experiment. A detector with mass ( $\sim 1$ Mton) would be a powerful tool for studying neutrino physics from atmospheric neutrinos. Thanks to the larger dimensions of the detector, higher energy neutrino-induced muons can be fully contained and their energy can be measured. Using the atmospheric neutrino flux, the distinctive oscillatory pattern as a function of L/E could be directly observed. The factor of 20 increase in detector fiducial mass will allow statistical improvements in all the topics studied and, perhaps, the emergence of new scientific topics. Other natural sources of neutrinos, such as lithospheric neutrinos, have not yet been studied extensively and could, in principle, be observed by the new detector concepts. An initial result in this area has recently been announced by KamLAND. Typically, the neutrino energies for these processes are below $10 \mathrm{MeV}$ and are sensitively dependent upon the low-energy threshold capability of the new detectors. The liquid scintillator detector concepts are likely to have the best opportunities for advancing these topics, but liquid argon detectors could also contribute.

Finally, we note that there may be galactic sources of neutrinos that are of lower energy and greater abundance than the ultra high-energy neutrino sources to be explored by detectors such 
as the Ice Cube Cherenkov detector now being constructed deep under the Antarctic ice sheet by an NSF sponsored collaboration. Galactic neutrinos have a natural source in inelastic nuclear collisions through the leptonic decays of charged secondary pions. This source is expected to be of comparable intensity and energy distribution to the high-energy photons that are born from neutral pion decays in the same collisions. Such neutrino sources, currently not detectable with SuperKamiokande, could be seen by a megaton-class neutrino detector that runs for several decades.

\subsection{Depth requirements for non-accelerator physics}

It is difficult to consider all possible non-accelerator physics channels and precisely predict the most optimum depth for either water Cherenkov detector or a liquid argon detector. The answer could easily depend on various technical assumptions, but it is certainly clear that depth comparable to or larger than present detectors (Super-Kamiokande is at $1000 \mathrm{~m}$ of rock or 2700 meter-water-equivalent depth) is needed for the best physics reach. A quantitative summary of depth considerations can be seen in [65].

Nucleon decay modes can be divided in two classes: ones where all of the nucleon energy is visible and ones where some of the nucleon energy escapes detection. In the first case, the total momentum and energy balance is a powerful tool for background reduction, and it has been often argued that these modes should require only modest shielding from cosmic rays. Indeed, most of the decay modes that were searched for in the first generation detectors required only modest depth. IMB operated successfully at a depth of 2000 feet. However, in a very large water Cherenkov detector, cosmics not only produce background, but also reduce the live-time of the experiment by keeping the detector occupied by frequent large energy deposits. If we require live-time to be more than $90 \%$, a shallow depth of few tens of meters appears sufficient. This conclusion does not include consideration of the data rate, which is continuous for non-accelerator physics, and could be unmanageably high near the surface. The requirement of a reasonable data rate $(<10 \mathrm{~Hz}$ of muons) increases the depth required to approximately the Super-Kamiokande depth.

For the second class of nucleon decays in a water Cherenkov detector, a low energy tag from dexcitation photons may need to be used (For example $p \rightarrow \bar{v} K^{+}$with a $\sim 6.3 \mathrm{MeV}$ gamma from ${ }^{15} N$ de-excitation followed by $K^{+} \rightarrow \mu^{+} v$ with lifetime of $12 \mathrm{~ns}$ ). These require low energy thresholds for photons. This is difficult with a background of fast-neutron (spallation products from muons in the detector or in the surrounding rock) induced low energy background events at shallow depths. Nevertheless, since the tagging photon is in-time to the main event (with time window of $<50 n s$ ), one could conclude that these events also may not require much more than Super-Kamiokande depths. A subclass of events are, however, subject to fast neutron backgrounds. As an example of this, the mode $n \rightarrow \bar{v} \bar{v} v$ can be searched for by observing the de-excitation of 
the residual nucleus. The proposed ultimate DUSEL depth (about 6500 mwe) would reduce the muon background by about a factor of 100 with respect to Super-Kamiokande and certainly help in the observation of these modes with a low energy component.

For a liquid argon calorimeter, much higher resolution may permit relaxation of these issues. In particular, the $\bar{v} K^{+}$mode could be much easier to detect because the kaon could be identified by its energy deposit ( $\mathrm{dE} / \mathrm{dx})$. Nevertheless, some minimum depth will very likely be necessary to reduce backgrounds from fast neutrons and to reduce the data rate to manageable levels.

For solar neutrinos in a water Cherenkov detector, the important issue is dead-time introduced by spallation induced fast neutron backgrounds. At Super-Kamiokande this dead-time is $\sim 20 \%$. To maintain the same level of dead-time for a much larger detector, depth similar to or greater than Super-Kamiokande (2700 mwe) will be needed. For a liquid argon detector, this requirement could be relaxed because the dead volume around a cosmic muon could be better defined.

For a supernova in our galaxy $(10 \mathrm{kpc})$, the signal level is so large $(\sim 10000 / \mathrm{sec}$ over a 10 sec burst), that the spallation background at depths as shallow as 500 mwe are manageable. For detection of supernova in neighboring Andromeda ( $\sim 750 \mathrm{kpc})$, however, greater depth $(>1300$ mwe) is needed. Optimizing depth for diffuse relic supernova neutrino search needs to take into account the deadtime loss as well as background from spallation products such as ${ }^{9} \mathrm{Li}$ which beta decays and then ejects a neutron. The analysis in [65] suggests that this search may require depths similar to Super-Kamiokande even if one could get the enhancement in signal to background from gadolinium loading.

In summary, the driving issues for depth consideration for future large water Cherenkov or liquid argon detectors will be backgrounds to low energy events from spallation products and data rates. If one wants to maintain sensitivity to specific important physics channels such as $p \rightarrow \bar{v} K^{+}$ in a water detector, and solar and supernova neutrinos in either technology, depth in the same range as the current Super-Kamiokande detector is needed. Greater depth will enhance the physics reach of the detector.

\section{RESULTS AND CONCLUSIONS}

The following summary results and conclusions were discussed and agreed to at the Sep. 17 2006 meeting of the study group.

- Very massive detectors with efficient fiducial mass of $>100$ kTon (in the case of water Cherenkov several hundred kTon and in the case of a liquid argon detector $\sim 100 \mathrm{kTon}$; for accelerator based neutrino physics these two would be roughly equivalent in sensitivity) could be key shared research facilities for the future particle, nuclear and astrophysics re- 
search programs. Such a detectors can be used with a long baseline neutrino beam from an accelerator laboratory to determine (or bound) leptonic $\mathrm{CP}$ violation and measure all parameters of 3 generation neutrino oscillations. At the same time, if located in a low background underground environment, they would have additional physics capabilities for proton decay and continuous observation of natural sources of neutrinos such as supernova or other astrophysical sources of neutrinos.

- The Phase-II program will need considerable upgrades to the current accelerator intensity from FNAL. Main Injector accelerator intensity upgrade to $\sim 700 \mathrm{~kW}$ (from the current $\sim 200 \mathrm{~kW}$ ) is already planned for Phase-I of the program (NOvA). A further upgrade to 1.2 MW is under design and discussion as described briefly in this report. The phase-II program could be carried out with the these planned upgrades. Any further improvements, perhaps with a new intense source of protons, will obviously increase the statistical sensitivity and measurement precision. Such an upgrade could significantly reduce the running times (especially in antineutrino mode) and increase statistical precision.

- A water Cherenkov detector of multi-100kTon size is needed to obtain sufficient statistical power to reach good sensitivity to $\mathrm{CP}$ violation. This requirement is independent of whether one uses the off-axis technique or the broadband technique in which the detector is housed in one of the DUSEL sites.

- High signal efficiency at high energies and excellent background reduction in a liquid argon TPC allows the size of such a detector to be smaller by a factor of 3 to 4 compared to a water Cherenkov detector for equal sensitivity. Such a detector is still quite large.

- The water Cherenkov technology is well-known. The issues of signal extraction and background reduction were discussed and documented at length in this study. The needed background reduction and energy resolution is achievable and well understood for the broadband beam approach, but not yet fully optimized. Key issues for scaling up the current generation of water Cherenkov detectors (Super-Kamiokande, SNO, etc.) and locating such detectors in underground locations in DUSEL have been investigated. The cost and schedule for such a detector could be created with high degree of confidence. A first approximation for this was reported to this study.

- For a very large liquid argon time projection detector key technical issues have been identified for the building of the detector. A possible development path includes understanding argon purity in large industrial tanks, mechanical and electronics issues associated with long wires, and construction of at least one prototype in the mass range of $1 \mathrm{kTon}$. 
- In the course of this study we have examined the surface operation of the proposed massive detectors for accelerator neutrino physics. Water Cherenkov detector are suitable for deep underground locations only. Surface or near surface operation of liquid argon TPCs is possible, but requires that adequate rejection of cosmic rays be demonstrated. Surface or near surface operation capability is essential for the off-axis program based on the existing NuMI beam-line because of the geographic area through which the beam travels.

- For an off-axis program based on the NuMI beam-line, baselines of about $800 \mathrm{~km}$ and offaxis distances of 10 to $40 \mathrm{~km}$ were considered for CP violation physics. Since the detector location is on the surface the best choice appears to be a fine grained detector such as a large liquid argon TPC. The scenarios considered for this program were: a) 100 kTon LArTPC at the 2nd oscillation maximum (40-60 mrad) in conjunction with the Phase I NOvA detector. b) 100 kTon LArTPC at the Phase I NOvA site. For scenario a) we find that the simple addition of a 2nd detector does not have significant sensitivity for CP. Scenario b) does have sensitivity as shown in Figure 24. A third scenario, using two detectors of $50 \mathrm{kTon}$ each at the first and second maximum has also been analysed (see Figure 26). Additional scenarios are presented in Table VIII.

- For a wideband program to DUSEL (either at Henderson (1495 km) or Homestake(1290 $\mathrm{km})$ ), two choices for detector technology were considered: a deep sited large water Cherenkov detector with fiducial mass of $\sim 300 \mathrm{kT}$ or a $100 \mathrm{kT}$ liquid argon TPC (which may be located either on the surface or underground). These were found to have good sensitivity for $\mathrm{CP}$ violation after exposure to the same amount of beam. The sensitivity for 1300 $\mathrm{km}$ location and its variations exposure are shown in Figures 11 to 22. The sensitivity was found to be about the same for $1495 \mathrm{~km}$.

- Baselines shorter than $500 \mathrm{~km}$ on the NuMI beamline from FNAL have severe technical limitations for performing the CP violation science because of the low energy of the oscillated events, difficulty of separating the ambiguities due to mass hierarchy, and the surface location of the massive detectors.

\subsection{Brief comparison of experimental approaches}

In the course of this year long study we have been able to draw several very clear conclusions. Regardless of which options evolve into a future program, the following will be required.

1. A proton source capable of delivering $1-2 \mathrm{MW}$ to the neutrino production target. 
2. Neutrino beam devices (targets and focusing horns) capable of efficient operation at high intensity.

3. Neutrino beam enclosures which provide the required level of environmental and personnel radiological protection.

4. Massive ( $>>100$ kton) detectors which have have high efficiency, resolution and background rejection.

5. For each of the above items, significant investment in $\mathrm{R}$ and/or $\mathrm{D}$ is required and needs to be an important aspect of the current program.

We have found that the main areas of this study can be discussed relatively simply if we divide them into two broad categories : 1) The neutrino beam configuration and 2) The detector technology. Further, we are able to summarize our conclusions in two tables which show the pros and cons of the various options.

In Table $\mathrm{X}$ we compare the pros and cons of using the existing NuMI beam and locating detectors at various locations, versus a new wide band neutrino beam, from Fermilab but directed to a new laboratory located at one of the potential DUSEL sites, i.e. at a baseline of 1300 to $2600 \mathrm{~km}$.

In Table XI we compare the pros and cons of constructing massive detectors ( 100 - $300 \mathrm{kT}$ total fiducial mass) using either water Cherenkov or liquid argon technology.

\subsection{Project timescales}

In the following we briefly comment on the possible timelines for the different components of the program we have described in the report. At this stage it is difficult to understand the funding, manpower, and other constraints to the program, therefore the study group has decided to comment only on technically driven schedules.

- The FNAL proton upgrade timeline: The SNuMI project which aims to upgrade the Fermilab accelerator complex to deliver higher intensity from the Main Injector, submitted a conceptual design report (CDR) in the fall of 2006. The timescale for the project will become clearer after the review process is completed. A preliminary timeline has been provided to this study. The complete upgrade will be carried out in two steps. In the first step, the recycler based upgrade (proton plan phase-II) will bring the total beam power to $700 \mathrm{~kW}$ by early 2011. In the second step, the accumulator upgrade (the complete SNuMI project) will bring the total intensity to 1.2 MW. An aggressive plan calls for performing the complete upgrade up to 1.2 MW by 2012. But this will depend on the outcome of reviews and discussions that will take place in the next year. 


\begin{tabular}{|c|c|c|}
\hline & Pro & Con \\
\hline NuMI On-axis & $\begin{array}{l}\text { Beam exists; } \\
\text { Tunable spectrum; }\end{array}$ & $\begin{array}{l}\mathrm{L} \sim 735 \mathrm{~km} \\
\text { Sensitivity to mass hierarchy is limited } \\
\text { Difficult to get flux }<3 \mathrm{GeV}\end{array}$ \\
\hline $\begin{array}{l}\text { NuMI Off-axis } \\
\text { (1st maximum) }\end{array}$ & $\begin{array}{l}\text { Beam exists ; } \\
\text { Optimized energy; } \\
\text { Optimized location for } \\
1 \text { st detector; } \\
\text { Site will exist from NOvA project; }\end{array}$ & $\begin{array}{l}\mathrm{L} \sim 800 \mathrm{~km} \\
\text { Limited sensitivity to mass hierarchy }\end{array}$ \\
\hline $\begin{array}{l}\text { NuMI Off-axis } \\
\text { (2nd maximum) }\end{array}$ & $\begin{array}{l}\text { Beam exists; } \\
\text { Optimized energy; } \\
\text { Improves mass hierarchy } \\
\text { sensitivity if } \theta_{13} \text { is large; }\end{array}$ & $\begin{array}{l}\mathrm{L} \sim 700-800 \mathrm{~km} ; \\
\text { Extremely low event rate; } \\
\text { A new site is needed; } \\
\text { Energy of events is } \sim 500 \mathrm{MeV} ; \\
\text { Spectrum is very narrow }\end{array}$ \\
\hline WBB to DUSEL & $\begin{array}{l}\text { More optimum (longer) baseline; } \\
\text { Can fit oscillation parameters } \\
\text { using energy spectrum; } \\
\text { Underground DUSEL site for detector; } \\
\text { Detector can be multi-purpose; }\end{array}$ & $\begin{array}{l}\text { New beam construction project }>\$ 100 \mathrm{M} \text {; } \\
\text { Multi-year beam construction; }\end{array}$ \\
\hline
\end{tabular}

TABLE X: Comparison of the existing NuMI beam to a possible new wide band low energy (WBLE) beam to DUSEL

- Construction of a new beam towards DUSEL: Construction of a possible new beam towards is not part of the SNuMI project. Only preliminary discussions, cost, and schedule estimates exists. The scope of the project is similar to the NuMI project which was described in Section 7.1. Based on the NuMI experience, a rough outline for the project could be: 1 to 2 years for preparation and geological site investigations, 2.5 to 3 years for civil construction, and 1 year for installation of technical systems: a total of 4.5 to 6 years for construction of the beam-line. There are a number of issues that are different between NuMI and a new beam-line to DUSEL. These are related to the greater downwards angle of the DUSEL beam-line and the proximity of the DUSEL beam-line to the FNAL site boundaries. These issues and their mitigation will be addressed in a separate note [52].

- Construction of a deep large water Cherenkov detector: There are two well recognized considerations that define the time scale over which a large water Cherenkov detector could 


\begin{tabular}{|c|c|c|}
\hline & Pro & Con \\
\hline $\begin{array}{l}\text { Water } \\
\text { Cherenkov }\end{array}$ & $\begin{array}{l}\text { New background rejection techniques } \\
\text { available; } \\
\text { Signal energy resolution } \sim 10 \% ; \\
\text { Underground location } \\
\text { makes it a multi-purpose detector; } \\
\text { Cosmic ray rate at } 5000 \mathrm{ft} \text { is } \sim 0.1 \mathrm{~Hz} \text {. } \\
\text { Excellent sensitivity to } p \rightarrow \pi^{0} e^{+}\end{array}$ & $\begin{array}{l}\text { Must operate underground; } \\
\text { Scale up factor is }<10 ; \\
\text { Cavern stability must be assured } \\
\text { and could add cost uncertainty; } \\
\text { NC background depends on spectrum } \\
\text { and comparable to instrinsic background; } \\
\text { Low } v_{e} \text { signal efficiency }(15-20 \%) ;\end{array}$ \\
\hline $\begin{array}{l}\text { Liquid } \\
\text { Argon } \\
\text { TPC }\end{array}$ & $\begin{array}{l}\text { Promises high efficiency and } \\
\text { background rejection; } \\
\text { Has potential to operate } \\
\text { on (or near) surface; } \\
\text { Could be placed on surface } \\
\text { either at NuMI Offaxis or DUSEL; }\end{array}$ & $\begin{array}{l}\text { Needs considerable R\&D for costing; } \\
\text { Not yet demonstrated by } \\
\text { simulation of a large detector; } \\
\text { Needs detailed safety design for } \\
\text { deep location in a cavern; } \\
\text { Needs detailed demonstration } \\
\text { of cosmic ray rejection; } \\
\text { Surface cosmic rate } ~ 500 \mathrm{kHz} ; \\
\text { Surface operation limits } \\
\text { physics program; }\end{array}$ \\
\hline
\end{tabular}

TABLE XI: Comparison of Water Cherenkov to Liquid Argon detector technologies

be built: the underground cavern construction and manufacturing of large numbers of photomultiplier tubes. For both the single cavern and the multiple cavern concepts of the detector a significant period of exploratory excavations and bore holes will be needed. After this period ( $\sim 1-2 \mathrm{yrs}$ ) approximately 5 to $6 \mathrm{yrs}$ of excavation is needed to reach the needed total volume. The PMT manufacturing period depends on the choice of the PMT, which is different for the two different concepts for the detector. For 20 inch PMTs, the UNO plan calls for manufacturing 56000 tubes in about 8-10 yrs. For the Homestake multiple module proposal, the plan calls for manufacturing 10-12 inch PMTs with a rate of about 150000 
tubes in 6-7 yrs. The collaborations are communicating with two large manufacturers of hemispherical PMTs. Preliminary conclusions are that each of the two manufactures have sufficient capacity currently to produce 10-12 inch diameter PMTs at about 1/2 the rate that is needed for these projects. For either choice, smaller or larger diameter, the production capacity needs to be enhanced to meet the need, but the investment needed in not considered extraordinary. There could be bottlenecks in production of materials (for example, glass) that need to be fully understood.

- Construction of a very large LARTPC: The cost and schedule estimate for a very large liquid argon TPC of size (50 to $100 \mathrm{kTon}$ ) must be preceded by a series of development steps. Although the viability of the technique has been established by the ICARUS group, a factor of 10 cost reduction is required to make a very large detector economically possible. The development program is outlined in [56] and contains three projects. One project involves techniques for the purification of liquid argon to achieve long electron drift times, low noise electronics design, and materials qualification. A second project is the construction of a $\sim 3$ ton module to test design concepts for the very large detector; and the third project is the design and construction of $\mathrm{a} \sim 1 \mathrm{kT}$ detector to be constructed using the techniques proposed for the very large detector.

The first project is in progress at FNAL and Yale. Long (many millisecond) electron drift lifetimes have been achieved and the project is expected to be complete by mid 2007. Dependent on funding the second project could produce results by the end of 2007 . The siting and mass of the detector to be proposed for the third project are under discussion. Once a choice is made, the group would like to start the design immediately. Completion of the design for project 3 is expected to take 1 year and requires successful completion of the other projects. A preliminary cost for project 3 at this time is $\sim \$ 10 \mathrm{M}$. The above program is essential for a LARTPC detector on the surface or underground. The cost implications for siting a very large detector at underground locations are being discussed, but they need further work.

\section{ACKNOWLEDGMENTS}

We are grateful for the support that the directorates of both Fermi National Accelerator Laboratory and Brookhaven National Laboratory have given to this work. The interaction with the NuSAG committee was also extremely important for setting the time table to finish the large number of calculations performed in this report. 


\section{APPENDIX A: ANSWERS TO QUESTIONS RAISED BY NUSAG}

1. Noting the existence of discrepant sensitivity calculations even for the same detector, it would be most useful to have any such calculations performed with consistent assumptions and methodologies.

a) Fixed, common, stated values of the mixing parameters not explicitly under study.

b) Common, stated and plotted, cross sections vs. En. Common, stated nuclear models.

c) Stated assumptions about energy resolution, background rejection.

d) If appropriate, common total p.o.t. If sensible, use a common proton energy and anti-nu running fraction. If not, state the optima chosen.

e) What methods are used to extract the oscillation parameters from the final event sample (counting? fitting the spectrum?)

f) Standardized, stated method for defining sensitivity.

2. Give sufficient detail in tables and/or plots to allow a reader to understand how the numbers for rates or sensitivities are obtained. We would expect that many of the results would be easily accessible to a physicist with a calculator. Here are some useful inputs that come to mind (meant as a guide only):

a) Specify the signal channel(s). (We will assume here that it is quasi-elastic.) b) What simple cuts (energy, etc.), if any, do you apply?

c) The number of INTRINSIC $v_{e}$ events reconstructed as signal, and their reconstructed energy spectrum (in reconstructed Enu(QE) or Evis, or Ee, or whatever you'll use.)

d) What is the purity of the QE selection, that is, for true $v_{e}$ events, what fraction of those selected as QE are actually QE (as a function of $\mathrm{E}$ )?

e) The total number of NC $\pi^{0}$ events, and spectra vs. true Enu and $\pi^{0}$ momentum.

f) The number of $\mathrm{NC} \pi^{0}$ events reconstructed as signal, and their reconstructed energy spectrum. What is the true Enu spectrum for the NC pi0 events reconstructed as signal?

g) The NC $\pi^{0}$ rejection assumed, as a function of... ( $\pi^{0}$ momentum?)

h) The assumed systematic errors on each of the backgrounds, with any relevant dependence on energy. How are these estimates arrived at?

i) The assumed signal efficiency as a function of energy. How are these estimates arrived at?

j) Provide tables and spectra (vs. true and reconstructed $E_{v}$ ) giving the initial population of events, before cuts, by process (QE, CCpi+, DIS,...), how these numbers diminish as the cuts are applied, and in the final sample at the various oscillation parameter test points. An entry at the 3-s sensitivity limit would be informative. Scatter-plots of reconstructed vs. true En for individual signal and background channels may be informative. 
3. Specify the level of simulation that goes into your currently-generated sensitivity estimates. For example:

a) How is energy resolution treated? Give a plot of the assumed energy resolution (electron energy and neutrino energy) vs. energy.

b) How is the selection of QE events treated?

c) How is the rejection of pi0's modeled?

We are grateful to the NUSAG committee to provide questions that could be used to guide the study. The report was written with the desire to answer these questions. Some of the details that these questions ask for are in the supporting documents which can be obtained from the study website: http://nwg.phy.bnl.gov/fnal-bnl/. To keep the length of the report minimum we have decided not to repeat the material that can be found in the body of the report.

4. What near detector location/size/technology/performance/cost is assumed/needed to achieve the assumed systematic errors?

In section 10.1 we have summarized the thoughts on the near detector issues for the Phase-II(B) DUSEL based broadband approach. The requirement on systematic error on the background are relatively modest $(10 \%)$. The harshest requirement might be on the energy scale systematic of $1 \%$ which is needed to achieve the best precision on the atmospheric parameters of $\Delta m_{32}^{2}$ and $\sin ^{2} 2 \theta_{23}$. The main technical issues for the near detector are the location for its deployment, the deviation from $1 / r^{2}$ behavior of the flux due to the close location of the detector, and the high event rate at the near site. The study did not look at these problems in detail. Fortunately, there is now rich experience on these issues from the running NuMI-MINOS experiment. Most of this experience can be applied directly to the future project.

For Phase-II(A) approach using the NuMI offaxis beam, the near detector requirements have not been studied. The location of such a detector could be in the existing tunnel that connects the NuMI near detector site to the beam tunnel. The study has not looked at the event rates or potential difficulties due to the deviation from both $1 / r^{2}$ behavior and from having a source with a wider angular acceptance at the near detector than the far detector.

5. If possible, for comparison purposes, use the same methodologies to make parallel sensitivity estimates for NoVA (single detector) and T2K. What sensitivity for NoVA do you calculate for the same number of p.o.t. assumed in question 1? Please see Section 13.2.

6. All sensitivity calculations for off-axis configurations must include events from neutrinos in the high-energy peak from kaon decay.

The detector performance criteria are in Section 13.2.

7. What detector technologies are still worth pursuing for a 2nd off-axis detector - Liquid scintillator? Water Cerenkov? Liquid Argon? Other?

Over the past several years, three potential detector technologies have been considered for a 
next generation experiment: liquid scintillator (similar to NOvA), water Cherenkov and a liquid argon TPC. Here, we summarize the conclusions which have been made to date in regard to the detector technology that would be best suited to the off-axis beam.

Studies of a massive liquid scintillator detector using the simulations developed for NOvA have shown that the backgrounds (mostly neutral current) would be approximately $1: 1$ with the signal at the second maximum and this option was not considered further.

A water Cherenkov detector of the size proposed for DUSEL could give sufficient rate in the $\mathrm{NuMI}$ beam, though there might again be a question of background rejection. However it has been concluded that this size of detector must be sited deep underground to avoid being swamped by cosmic ray muons and there is no existing deep site available along the NuMI beam, and so we do not consider this a viable option.

A liquid argon TPC has the advantages of high efficiency and high background rejection for neutral current events, using the high spatial resolution. Thus for the same sensitivity in the same beam it can be factors of around 3 smaller than a water Cherenkov detector. For the sensitivity studies we have assumed liquid argon detector(s) with a total fiducial mass of 100 kTon.

8. There were several references to the possibility of a detector at $\sim 250 \mathrm{~km}$ in the $\mathrm{NuMI}$ beam. Is this being pursued by the Working Group? What are the general properties of this approach?

Shorter baseline lengths for NuMI off-axis detectors have been considered in the literature [26]. For example, for a baseline of $250 \mathrm{~km}$, the first and second oscillation maxima are at 0.50 $\mathrm{GeV}$ and $0.17 \mathrm{GeV}$, respectively. There are two reasons for considering shorter baselines: small matter effects and larger numbers of events because of the closer distance. This solution, however has several difficulties. The main ones are: i) The low energies needed forces us to consider large off-axis angles ( $>40 \mathrm{mrad}$ ) where the flux of neutrinos is rather poor and the contamination from high energy neutrinos from kaon decay large. This largely negates the advantages of the larger flux because of the closer distance. The event rate can be easily obtained from [5] by scaling. ii) Natural choice for a detector at these energies is a water Cherenkov counter. Since most of the events at these energies are quasi-elastics for which a water Cherenkov detector has good efficiency, little is gained by utilizing a liquid argon TPC. The water Cherenkov detectors needed are too large for operation on the surface as explained in Section 12. iii) For the first oscillation maximum, an experiment with almost identical parameters is already being carried out in Japan (T2K). Combining the results of T2K and Phase-I of the US program is a subject of various reviews[66].

9. Provide cost and schedule estimates for the same fiducial mass and PMT coverage/channel count used for sensitivity estimates. (We realize that fiducial/total mass ratios may be hard to estimate, but the assumptions should be stated.) 
We hope that the committee understands that the work reported in this study was carried out in parallel in a very short period of time. In addition, members of the study group are considering several options for detector sites and design. Therefore it is difficult to obtain complete consistency in the assumptions that went into simulations versus detector design and cost estimates, etc. Obviously we will do the best we can to point out the various points of departure and will depend on good judgment.

The design and cost for a detector in the Henderson laboratory were provided in the presentation of Prof. Chang kee Jung at [49]. The fiducial volume for UNO was quoted to be $440 \mathrm{kT}$ at a preliminary cost of $\$ 437 \mathrm{M}$.

A conceptual detector design for $300 \mathrm{kT}$ for Homestake was presented to the committee in [50]. The authors of that report provided the following answer for their choices:

A single 100 kiloton module will have a cylindrical fiducial volume with a diameter of 50 meters and a height of 50 meters. The PMTs on the vertical face of the cylinder will have their photo-cathodes on the surface of a 52 meter diameter cylinder. The top and bottom PMTs will be separated by 52 meters. This layout defines a fiducial volume that begins 1 meter inside the PMT photo-cathode surface. In addition, there will be 0.5 meter veto region surrounding the entire detector so that the chamber walls will be on a 53 meter diameter cylinder.

Our budget estimate for the excavation of the detector chamber was based on a 50 meter diameter by 50 meter high cylinder. The change from 50 meter to 53 meters involves a volume increase of $18 \%$ and a surface area increase of $12 \%$. Although our budget breakdown details permit us to apply the above scale factors to each of the volume and surface area budget items, we decided, for this answer to merely use an average cost increase of $15 \%$. When applied to a single module, the construction cost increases from $\$ 29.1$ million to $\$ 33.5$ million. Note, that these numbers include a contingency of $30 \%$. The total single 100 kiloton detector cost increases from $\$ 116.6$ million to $\$ 121$ million, an increase of $3.6 \%$.

Similarly, when this cost increase factor is applied to three detectors, the three chamber cost increases from $\$ 66.1$ million to $\$ 76$ million and the total three detector cost increases from $\$ 308.9$ million to $\$ 318.8$ million.

In the above we not included the effect of moving the PMTs from the original 50 meter diameter cylinder to a 52 meter diameter cylinder, a surface area increase of $8 \%$. If apply this factor to the previously assumed PMT and associated electronics cost of \$62.1 million this creates another $\$ 5$ million increase per 100 kiloton detector. The final cost including all contingencies is then $\$ 126$ million for a single 100 kiloton detector and $\$ 323.8$ million for three such detectors. The above increase is less than $10 \%$ for budget that has a contingency of about $34 \%$.

Finally, the simulations for the background estimates were reported in [30]. They were performed with the exact geometry of the Super-Kamiokande detector (with 40\% PMT coverage 
using 20 inch diameter tubes). The Homestake detector cost is for 11 inch tubes and $25 \%$ coverage. We are confident that this coverage is sufficient because of several factors. First, the PMT information both Hamamatsu and Photonis shows that smaller diameter semi-hemispheric tubes have higher quantum efficiency $(\mathrm{QE})$ than the 20 inch tube (for example, the Hamamatsu 10.5 inch tube has QE of $25 \%$ and the 20 inch tubes has QE of $20 \%$ at $390 \mathrm{~nm}$ ) This difference is apparently well-known and documented. Secondly, the collection efficiency (efficiency of collecting the photo-electron into the dynode structure) is also known to be larger for the smaller diameter tubes. The collection efficiency factor (an increase of about $13 \%$ for the 10.5 inch tubes versus the 20 inch) is not well documented. Therefore, if corrected by these two effects, the $25 \%$ coverage with smaller 10.5 inch tubes corresponds to $35 \%$ coverage $\left(\sim 25 \% \times \frac{0.25}{0.20} \times 1.13\right)$ with the 20 inch photomultiplier tubes. We also expect that with a larger detector and far larger granularity, the background rejection will get better requiring less total coverage. Nevertheless, we understand that all of the above has to be demonstrated with benchtop measurements and detailed simulations for which we would like to ask for substantial $R \& D$ funds. If we must increase the coverage to $40 \%$ to achieve the physics goals then the cost increase will be approximately $\$ 112 \mathrm{M}$ which is certainly beyond the contingency we have allowed at this point.

10. For the modular water Cherenkov approach, are you defining 3 modules as your baseline detector?

The authors of report [50] reply:

"Yes, there are three main reasons we believe 3 modules is an optimum choice to start with. First, because of the long running times possible at FNAL it appears that a 300 kTon fiducial mass is sufficient to reach the desired sensitivity for neutrino oscillations. Second, it is clear that for proton decay searches a larger detector is needed, but for current background projections a few background events are expected in favored decay modes after exposure of 1 MT-yr. We believe that proton decay searches will benefit from further detector and analysis improvements after reaching this level of sensitivity. Any modules built after the first 3 modules will benefit from this knowledge. Third, there is considerable cost saving by starting the simultaneous construction of 3 cavities in the region of relatively well-known Homestake rock near the Ray Davis Chlorine chamber as explained in [50]."

11. For the water Cherenkov counters, we will be eager to hear of progress in algorithms for rejecting $\pi^{0} \mathrm{~s}$ (and the testing of them). What is the increase in $\pi^{0}$ rejection over that achieved by Super-K (as a function of $\pi^{0}$ energy) assumed in your current calculations? What have you reached with your own simulations/algorithms? Describe briefly the algorithmic improvements. Does this rejection depend more on total photo-cathode coverage, or on granularity?

The detailed account of the $\pi^{0}$ rejection is described in the accompanying report by C. Yanag- 
isawa et al. [31] as well as in the presentations by Yanagisawa and Dufour [30, 32]. It is also summarized in section 10.1.

The improvement to the signal to background depends on the neutrino spectrum and neutrino oscillation parameters. In the following we use the same neutrino energy spectrum and the same neutrino oscillation parameters as used in the above report with the $\mathrm{CP}$ violating phase of 45 degrees.

For the baseline of $1480 \mathrm{~km}$ (Fermilab to Henderson), using the new algorithm the signal to the background ratio can be improved from 0.30 to 1.9 , while retaining $40 \%$ of the signal events accepted by the current Super-Kamiokande algorithm. For the baseline of $2540 \mathrm{~km}$ (BNL to Homestake), using the new algorithm the signal to the background ratio can be improved from 0.35 to 2.1, while retaining $40 \%$ of the signal events accepted by the current Super-Kamiokande algorithm.

Dependence of these results above on the granuarity and photocathode coverage has not been studied in a systematic fashion, as we extensively used Super-Kamiokande-I (photocathode coverage of $40 \%$ ) Monte Carlo sample. It is also found that, given the $40 \%$ photocathode coverage, the signal to background ratio can be significantly improved for neutrino events with reconstructed neutrino energy of $<1.2 \mathrm{GeV}$ for a detector with better granularity. Other observations concerning this issue are touched upon in section 10.1.

\section{Though the worldwide community of proponents of large water Cerenkov detectors} seems to cooperate in simulations, algorithms, etc., we do not see evidence that there is any global planning (site-independent design studies or physics programs, etc.) underway for such a detector. Please comment.

One of the most useful results of the NUSAG process has been the cooperation in simulations and algorithms for large water Cherenkov detectors. This cooperation was most evident in the participation from the T2KK group in our discussions. We have also had fruitful interactions with the proponents of the Frejus based water Cherenkov detectors.

The description and calculations for the water Cherenkov approach in this report was a result of cooperation between two US based groups: the UNO group that wants to develop a single very large cavern for the detector and the Homestake based group which wants to develop the detector in multiple modules. Both groups have worked together to understand and suppress the backgrounds in the detector and also have settled on a similar physics strategy for addressing $\mathrm{CP}$ violation in neutrino oscillations.

There are currently 5 well considered proposals for a very large water Cherenkov detector worldwide: Hyper-kamiokande detector in Japan, a possible detector in Korea on the same neutrino beamline as JPARC to Super-Kamiokande, a very large detector in the Frejus laboratory in France, and the two possible sites for DUSEL (Homestake or Henderson mines) in the U.S with either a large single volume detector(UNO) or a detector in multiple modules such as the Homestake 
proposal. A long baseline neutrino oscillation program with emphasis on reaching sensitivity to $\mathrm{CP}$ violation in neutrino mixing is central to all these proposals. Therefore we believe there is good cooperation and agreement on the issue of the physics program for such a detector.

The other two factors for such a detector are a) site development, and b) photo-sensor and electronics R\&D and acquisition. The site development is a very large part of this detector design, and therefore must be handled locally. There is cooperation and communication between these groups to compare costs and schedule for the site development. The costs and schedules appear understandable after considering the differences between the engineering and accounting practices in these geographic region, but we do not see how global site independent planning can be performed here. The photo-sensor and electronics R\&D is the dominant item in these projects. For the photo-sensor R\&D we agree that good cooperation could be helpful and lower the costs for everyone.

It should be remarked that each of the above geographical regions has a unique virtue for locating this massive detector. For Frejus, it is the availability of CERN as a neutrino source and the deep location next to the Frejus highway tunnel. For both HyperK and the Korea based detectors the uniqueness lies in the location on an existing neutrino beamline from JPARC. For the US sites the uniqueness is in first the distance available from Fermilab or BNL $(>1000 \mathrm{~km})$ which is now recognized as essential for performing the next generation experiment with large $\mathrm{CP}$ and matter effects, and second the depth available at the potential DUSEL sites to suppress cosmogenic backgrounds. Finally, the size of the detector projects are large but at a scale that could be contemplated on a national level. Therefore, global planning for a single such detector and site independent studies (in the manner of a very large accelerator project), is perhaps not warranted.

For Liquid Argon:

These questions were answered by the liquid argon subgroup. The answers were coordinated by Prof. Bonnie Fleming.

NuSAG recommends that the Liquid Argon group reweight its emphasis from sensitivity/reconstruction/pattern recognition to hardware issues and cost estimates. We realize that a full switch cannot occur if the LAr group is a big part of the more generic off-axis calculations in the Working Group, but, for example, LAr-specific reconstruction and particle ID algorithms seem less pressing than technical feasibility.

13. What has actually been measured on purity of the Ar in a tank made with industrial technology? If not yet tried, when will the first tests be?

Response: No tests have yet been performed on purity of Argon in a tank made with the industrial technology necessary for construction of a massive detector. This test will require a large tank, $\sim 1$ kTon, constructed using the same techniques as a large detector. This project has been envisaged by the LArTPC group as outlined in their report to NuSAG in 2005. A specific plan 
for this component of the R\&D path is presently under study and expected to converge within a year. In the meantime, small scale tests using the Materials Test Stand at Fermilab (see writeup for details) will have first results addressing purity issues within this year.

14. When do you expect to have tried 3-m drifts and long wires in the US? What effect will the capacitance of very long wires have on electronic noise?

Response: A program to study $5 \mathrm{~m}$ drifts using a prototype vessel at Fermilab is in the design stages. Depending on funding, results from this project are expected within the next two years. As well, long drift tests are underway in Europe on the same timescale.

A 30m long wire with 4 meters of interconnecting cable to electronics will have a capacitance of $620 \mathrm{pF}$ [43]. Using commercial amplifiers, a signal to noise of $\sim 9$ can be achieved, adequate for LArTPCs. Another configuration that has been considered is to use cold electronics, eliminating the interconnecting cable. This option is under study at Michigan State University in Carl Bromberg's group.

15. What are the R\&D milestones, with an estimated schedule, that would lead to a first realistic cost estimate for a detector of the 2 nd-off-axis or wide-band class?

Response: Before developing a realistic cost estimate for a massive detector, 50-100kTons in size, a reasonably sized, scaled down version of the massive detector should be constructed and operated. This detector will test purity in a vessel constructed using the same industrial techniques envisaged for the large detector, electronics, ability to handle cosmic ray rate, and cellular design. As well, smaller scale tests such as the $5 \mathrm{~m}$ drift test, long wires test etc, as described in the summary document, are necessary. However, it is the $1 \mathrm{kTon}$ scale test that drives the schedule. The schedule for this project is not yet fully fleshed out. 


\section{APPENDIX B: NUSAG CHARGE}

The charge letter is reproduced on the next two pages 


\title{
U.S. Department of Energy and the National Science Foundation
}

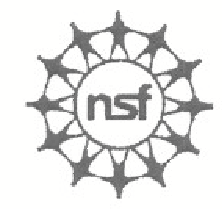

\author{
March 3, 2006
}

Professor Eugene Beier

Co-Chair, NuSAG

University of Pennsylvania

209 South 33rd Street

Philadelphia, PA 19104
Professor Peter Meyers

Co-Chair, NuSAG

Princeton University

306 Jadwin Hall

Princeton, NJ 08544

Dear Professors Beier and Meyers:

We would like to thank you and the Neutrino Scientific Assessment Group (NuSAG) for your timely and thoughtful responses to the initial questions that were posed to you, concerning neutrinoless double beta decay, reactor experiments and accelerator-based experiments to determine fundamental neutrino properties. They have already been very useful and will help us put together a strong US program in neutrino physics.

We would now like your group to address the APS Study's recommendation for a next-generation neutrino beam and detector configurations. Assuming a megawatt class proton accelerator as a neutrino source, please answer the following questions for accelerator-detector configurations including those needed for a multi-phase off-axis program and a very-long-baseline broad-band program. This assessment will be used as one of the key elements to guide the direction and timeline of such a possible next generation neutrino beam facility.

In your assessment, NuSAG should look at the scientific potential of the facility, the timeliness of its scientific output, and its place in the broad international context. Specifically:

- Scientific potential: What are the important physics questions that can be addressed at the envisioned neutrino beam facility?

- Associated detector options: What are the associated detector options which might be needed to fully realize the envisioned physics potentials? What are the rough cost ranges for these detector options?

- Optimal timeline: What would be the optimal construction and operation timeline for each accelerator-detector configuration, taking the international context into account?

- Other scientific considerations: What other scientific considerations, such as results from other neutrino experiments, will be important in order to optimally determine the design parameters? What would be additional important physics questions that can be addressed in the same detector(s)?

The DOE and the NSF would like a preliminary draft of your report by December 2006, with a final version by February 2007. 
Thank you in advance for your dedication to addressing these important and challenging questions.

Sincerely,

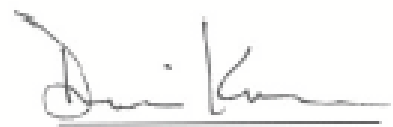

Dennis Kovar Associate Director Ottice of Nuclear Physics Department of Energy

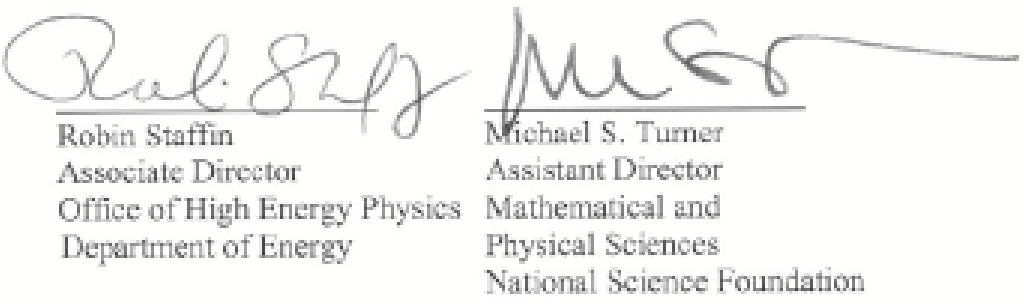




\section{APPENDIX C: CHARGE TO THIS WORKING GROUP}

April 5, 2006

Dear Colleague,

This letter is being sent to you as a follow-up to the Long Baseline Workshop held at Fermilab on March 6-7. This mailing list is composed of those who attended the study and signed up to receive further information or have subsequently expressed interest in the study. Since the kick off meeting we have redrafted the goals of the study. We have inserted a time scale which we judge to be achievable. The is goal is described in the attached document. You can anticipate that within days you will get a further document in which Milind Diwan and Regina Rameika have attempted to parse the study goals into a set of work packages. We would like to hear from people who are prepared to do some work on these issues. Especially we would be very happy to hear from people new to these studies.

However, as you might expect we do have some likely suspects in mind and Gina and Milind will be contacting people to help. Finally, we will also be recruiting an Organising/Advisory Committee to help us guide this study. We look forward to seeing progress on this study and would welcome your suggestions for additions, adjustments and approach .

With Best Regards,

Sally \& Mont

Sally Dawson, Chair, Physics Department, Brookhaven National Laboratory. Hugh Montgomery, Associate Director, Fermi Natinal Accelerator Laboratory 


\section{U.S. Long Baseline Neutrino Experiment Study}

April 3, 2006

\section{Preamble:}

The Fermilab Proton Driver (FPD) Study conducted in 2004/05 concluded that Neutrino Oscillation physics provides the main motivation for the FPD, and that the physics case is strong. In addition, the Fermilab $8 \mathrm{GeV}$ linac beam could support other physics experiments in parallel with an upgraded NuMI-based neutrino program. The study results were presented to the Fermilab PAC in the 2005 Aspen meeting, and were well received. However, the PAC expressed interest in the possibility of a further generation of neutrino oscillation experiments at a FPD (beyond NOvA) but anchored by the NuMI facility.

Brookhaven National Laboratory has considered in some detail a very long baseline neutrino oscillations (VLBNO) concept using an on-axis, wide band beam but with a very large detector at a longer distance. This beam could originate from either Fermilab or BNL. This approach requires a large underground detector presumably located at the NSF's planned DUSEL facility, which would also have potential for other frontier physics in addition to neutrino oscillation physics.

While these two approaches have a common goal of understanding neutrino masses and mixings, they are clearly different. We would like to have a thorough study and exploration of the differences and potential of the two approaches. To that end we have drafted a charge for a joint Fermilab/BNL study, the results of which could form the basis for a national program in neutrino physics. This charge follows an initial short workshop which laid out some of the issues:

http://www.fnal.gov/directorate/DirReviews/Neutrino_Wrkshp.html

\section{Timescale:}

The United States neutrino community is heavily engaged in operation and analysis of its existing program. On the other hand there are active discussions within advisory bodies and the agencies with a view to setting directions for future facilities inside the next year.

It would be desirable to see results of this U.S. Long Baseline Neutrino Experiment Study before October 2006, with a preliminary report by July 15, 2006.

\section{U.S. Long Baseline Neutrino Experiment Study}

Compare the neutrino oscillation physics potential of:

1. A broad-band proposal using a either an upgraded beam of around $1 \mathrm{MW}$ from the current Fermilab accelerator complex or a future Fermilab Proton Driver neutrino beam aimed at a DUSEL-based detector. Compare these results with those previously obtained for a high intensity beam from BNL to DUSEL.

2. Off-Axis next generation options using a 1-2 MW neutrino beam from Fermilab and a liquid argon detector at either DUSEL or as a second detector for the Nova experiment.

Considerations of each should include:

i) As a function of $\theta_{13}$, the ability to establish a finite $\theta_{13}$, determine the mass hierarchy, and search for CP violation and, for each measurement, the limiting systematic uncertainties.

ii) The precision with which each of the oscillation parameters can be measured and the ability to therefore discriminate between neutrino mass models.

iii) Experiment Design Concepts including:

Optimum proton beam energy

Optimum geometries

Detector Technology

Cost Guesstimate 


\section{APPENDIX D: STUDY GROUP MEMBERSHIP}

Chairs

\begin{tabular}{l|l|l}
\hline Sarah Dawson (co-chair) & Brookhaven National Lab. & dawson@bnl.gov \\
Hugh Montgomery (co-chair) & Fermi National Accelerator Lab. & mont@ fnal.gov \\
\hline
\end{tabular}

International Advisory Group

\begin{tabular}{l|l|l}
\hline Milind Diwan (co-leader) & Brookhaven National Lab. & diwan@ bnl.gov \\
Regina Rameika (co-leader) & Fermi National Accelerator Lab. & rameika@ fnal.gov \\
Joshua Klein & University of Texas & jrk@ mail.hep.utexas.edu \\
Franco Cervelli & INFN, Pisa & franco.cervelli@ pi.infn.it \\
Maury Goodman & Argon National Lab. & maury.goodman@ANL.GOV \\
Bonnie Fleming & Yale University & Konnie.fleming@yale.edu \\
Karsten Heeger & Lawrence Berkeley Lab. & Feeger@LBL.GOV \\
Steven Parke & Fermi National Acc. Lab. & parke@FNAL.GOV \\
Takaaki Kajita & kniversity of Tokyo & kajita@suketto.icrr.u-tokyo.ac.jp \\
\hline
\end{tabular}

A full list of participants is available at http://nwg.phy.bnl.gov/diwan/nwg/fnal-bnl/folks.txt 


\section{APPENDIX E: RELEVANT RESOURCES AND URLS FOR THE STUDY GROUP}

Main websites for this study group are:

http://home.fnal.gov/ rameika/LBL_Study/LBL_mainframe.htm

http://nwg.phy.bnl.gov/ diwan/nwg/fnal-bnl/

Additional materials can be found at:

http://www.fnal.gov/directorate/DirReviews/Neutrino_Wrkshp.html

http://www.hep.net/nusag_pub/May2006talks.html

http://www-numi.fnal.gov/

http://nwg.phy.bnl.gov/

http://www-lartpc.fnal.gov/LBStudy_LAr/2006LB.html

http://www.dusel.org/

http://www.lbl.gov/nsd/homestake/

http://nngroup.physics.sunysb.edu/husep/ 


\section{APPENDIX F: SCHEDULE OF MEETINGS AND REPORT PREPARATION}

Most of the work of the working group was carried out by small subgroups that worked on the

individual documents. The work was mostly carried out by email and telephone. The following meetings were very helpful for wider interactions.

November 14, 2005 FNAL/BNL meeting to explore collaboration, BNL

March 3, 2006

Charge letter for NuSAG to examine APS study recommendation for a next generation neutrino beam and detector configuration

March 6-7, 2006

First kick-off workshop for organization of the study at FNAL

April 5, 2006

Charge letter to the study from Dawson and Montgomery

April 11, 2006

Preparation of the task list and assignments

May 20, 2006

Presentations to NuSAG committee about the study in Chicago

June 27-28, 2006

Second workshop on detector technologies at FNAL

July 6, 2006

Status report to HEPAP from NuSAG, presentation by P. Meyers, HEPAP meeting in Washington D.C.

July 15, 2006 Deadline for preparation of individual reports from the task list

September 16-17, 2006 Third workshop on preparation of the joint summary report

October 16, 2006 Deadline for presentation of the joint report

December, 2006 Deadline of report from NuSAG to HEPAP

Other meetings of note where interactions took place are

June 13-19, 2006 Neutrino 2006, Conference in Santa Fe

July 11-21, 2006 Neutrino Physics with Liquid Argon TPCs, Yale Univ.

August 24-30, 2006 NuFact 06 Workshop, UC/Irvine

Sep 21-23, 2006 NNN06, University of Washington

March 29-30, 2007 Fermilab Physics Advisory Meeting 
[1] Neutrinos and Beyond: New Windows on Nature, Neutrino Facilities Assesment Committee, National Research Council, (2003), ISBN-0-309-087 16-3, http://www.nap.edu/catalog/10583.html.

[2] The Physics of the Universe, a Strategic Plan for Federal Research at the Intersection of Physics and Astronomy, National Science and Technology Council Committee on Science, February 2004, http://www.ostp.gov/html/physicsoftheuniverse2.pdf .

[3] FY2007 Administration Research and Development Budget Priorities, J.H. Marburger, III and J.B. Bolten, Executive Office of the President Memorandum, July 8, 2005, http://www.ostp.gov/html/budget/2007/ostp_omb_guidancememo_FY07.pdf .

[4] [MINOS Collaboration], "Observation of muon neutrino disappearance with the MINOS detectors and the NuMI neutrino beam," arXiv:hep-ex/0607088.

[5] 'Event Rates for Off Axis NuMI Experiments," B. Viren, June 8, 2006. BNL-76869-2006-IR. hepex/0608059

[6] For a review of the low to medium energy neutrino cross sections see S. Zeller, talk at the NO-VE workshop, Feb. 7, 2006, Venice, Italy, http://nwg.phy.bnl.gov/diwan/nwg/fnal-bnl/docs/zeller-nove-feb06.pdf

[7] 'Simulation of a Wide-band Low-Energy Neutrino Beam for Very Long Baseline Neutrino Oscillation Experiments,” M. Bishai, J. Heim, C. Lewis, A. D. Marino, B. Viren, F. Yumiceva, July 20, 2006, BNL-76997-2006-IR.

[8] http://nwg.phy.bnl.gov/fnal-bnl

[9] W. Marciano, hep-ph/0108181, 22 Aug. 2001.

[10] M. Diwan et al., Phys. Rev. D 68, 012002 (2003) [arXiv:hep-ph/0303081].

[11] Stephen Parke, Talk in the HQL04 conference, Puerto Rico, June 1-June5, 2004.

[12] M. V. Diwan, Talk in the HQL04 conference, Puerto Rico, June 1-June5, 2004. arXiv:hep-ex/0407047.

[13] For a review see, V. Barger, D. Marfatia and K. Whisnant, Int. J. Mod. Phys. E 12, 569 (2003) [arXiv:hep-ph/0308123].

[14] M. Ishitsuka, T. Kajita, H. Minakata and H. Nunokawa, 'Resolving neutrino mass hierarchy and CP degeneracy by two identical detectors with different baselines," Phys. Rev. D 72, 033003 (2005) [arXiv:hep-ph/0504026].

[15] V. Barger, D. Marfatia and K. Whisnant, Phys. Rev. D 65, 073023 (2002) [arXiv:hep-ph/0112119].

[16] SNuMI conceptual design report, D. Bogert et al., 9 Nov. 2006, ProtonPlan2-doc-101. http://beamdocs.fnal.gov/SNuMI-public/DocDB/ShowDocument?docid=101

[17] 'Fermilab Proton Projections for Long-Baseline Neutrino Beams," Robert Zwaska for the SN- 
uMI planning group, July 17, 2006. FNAL-Beams-DOC-2393. http://beamdocs.fnal.gov/ADpublic/DocDB/ShowDocument?docid=2393

[18] D. Mcginnis, Beams Document 1782-v7, FNAL, 2005.

[19] R. Alber, et al., Proton Driver Study Group FNAL-TM-2136, FNAL-TM-2169. http://wwwbd.fnal.gov/pdriver/

[20] “Accelerator and Beam Requirements," A. Marchionni, Talk to the NuSAG committee, May 20, 2006. http://www.hep.net/nusag_pub/May2006/marchionni_NuSAG_May_06.ppt

[21] This document is still in revision. It describes the technical requirements and feasibility for a solid target with a 1MW capability. 'Target System for a Long Baseline Neutrino Beam," N. Simos, H. Kirk, J. Gallardo, S. Kahn, N. Mokhov. June 26, 2006. http://nwg.phy.bnl.gov/ diwan/nwg/fnal-bnl/docs/BNL-FNAL_Target_DRAFT.doc

[22] 'The AGS-Based Super Neutrino Beam Facility Conceptual Design Report," J. Alessi, et al., October 1, 2004, BNL-73210-2004-IR http://nwg.phy.bnl.gov/papers/agsnbcdr1.pdf

[23] NuMI Beam Characteristics, J. Hylen, Talk at the Workshop on Long Baseline Neutrino Experiments, March 6-7, 2006, Fermilab, USA. http://www.fnal.gov/directorate/DirReviews/Neutrino_Wrkshp_files/Hylen.pdf

[24] Dr. Dixon bogert, Communication in the course of this study.

[25] M. Bishai, Talk from the Sep 16-17 meeting of the U.S. long baseline experiment study, Sep 16, 2006 http://nwg.phy.bnl.gov/ diwan/nwg/fnal-bnl/sep16-17-2006/fnal_bnl_091606-bishai.pdf

[26] Determining the neutrino mass hierarchy and CP violation in NoVA with a second off-axis detector. Olga Mena (Fermilab), Sergio Palomares-Ruiz (Vanderbilt U.), Silvia Pascoli (CERN and Durham U., IPPP) . CERN-PH-TH-2005-195, IPPP-05-63, DCPT-05-126, FERMILAB-PUB-05-461-T, Oct 2005. 20pp. Published in Phys.Rev.D73:073007,2006 e-Print Archive: hep-ph/0510182

[27] Y. Fukuda et al., Nucl. Instrum. Meth. A 501, 418 (2003).

[28] Syunsuke Kasuga, Ph.D. Thesis, January 1998, University of Tokyo. http://nwg.phy.bnl.gov/ diwan/nwg/fnal-bnl/docs/kasuga.ps.gz

[29] S. L. Adler, Annals Phys. 50, 189 (1968); D. Rein and L. M. Sehgal, Annals Phys. 133, 79 (1981).

[30] C. Yanagisawa, Talk at Workshop on Long Baseline Neutrino Experiments, Fermilab, March 6-7, 2006. http://www.fnal.gov/directorate/DirReviews/Neutrino_Wrkshp.html

[31] C. Yanagisawa, C.. Jung, P.T. Lee, and B. Viren, Preprint submitted to The U.S. Long Baseline Neutrino Experiment Study. http://nwg.phy.bnl.gov/ diwan/nwg/fnal-bnl/docs/numunuePREBWv3.pdf

[32] Fanny Dufour and Edward Kearns, Talk at the Sep 16-17 meeting of the study group. http://nwg.phy.bnl.gov/ diwan/nwg/fnal-bnl/sep16-17-2006/060914-fnal-workshop.pdf

[33] The results for efficiency given in references [30] or [31] are with respect to visible events. Most of the 
NC elastic events are invisible in a water Cherenkov detector. We have adjusted the efficiency in table VI for this.

[34] C. Yanagisawa, Talk to this study group on Sep 16-17, 2006. http://nwg.phy.bnl.gov/ diwan/nwg/fnalbnl/sep16-17-2006/yanagisawa-fnal-sep16-06.pdf

[35] P. Huber, M. Lindner and W. Winter, Comput. Phys. Commun. 167, 195 (2005) [arXiv:hepph/0407333].

[36] T. Ypsilantis, A. Gougas, D. Hatzifotiadou and J. Seguinot, "A Long baseline RICH with a 27-kton water target and radiator for detection

[37] P. Glassel, "The limits of the ring image Cherenkov technique," Nucl. Instrum. Meth. A 433, 17 (1999).

[38] P. Antonioli et al., 'The AQUA-RICH atmospheric neutrino experiment," Nucl. Instrum. Meth. A 433, 104 (1999).

[39] E. V. Bugaev, Z. A. M. Zhilkibaev and M. D. Galperin, 'Propagation Of Cerenkov Radiation Theough Water,"Nucl. Instrum. Meth. A 248, 219 (1986).

[40] M. Shiozawa [Super-Kamiokande Collaboration], 'Reconstruction algorithms in the SuperKamiokande large water Cherenkov detector,”Nucl. Instrum. Meth. A 433, 240 (1999).

[41] Fanny Dufour, Talk at the T2KK www-rccn.icrr.u-tokyo.ac.jp/workshop/T2KK06/files/19_060714fdufour2.pdf

[42] B. Viren, NUMI internal note 719. July 6, 2001. http://nwg.phy.bnl.gov/diwan/nwg/fnalbnl/docs/numi0719.ps.gz

[43] C. Bromberg et al., "A Large Liquid Argon Time Projection Chamber for Long-Baseline, Off-Axis Neutrino Oscillation Physics with the NuMI Beam". Submitted to the DOE NuSAG committee. Fermilab-FN-0776-e, 2005. http://www-lartpc.fnal.gov

[44] Conceptual design of a scalable multi-kton superconducting magnetized liquid Argon TPC. A. Ereditato (INFN, Naples), A. Rubbia (Zurich, ETH) . Oct 2005. 4pp.Invited talk at 7th International Workshop on Neutrino Factories and Superbeams (NuFact 05), Frascati, Italy, 21-26 Jun 2005. Published in Nucl.Phys.Proc.Suppl.155:233-236,2006. e-Print Archive: hep-ph/0510131

[45] A. Curioni, 'Monte Carlo Study of an LArTPC for Neutrino Experiments". Writeup for the FNAL/BNL Long Baseline Study. http://nwg.phy.bnl.gov/ diwan/nwg/fnalbnl/docs/curioni_JulyTN.pdf

[46] S. Linden, 'Simulations", Workshop on Neutrino Physics with Liquid Argon TPC Detectors, http://www-lartpc.fnal.gov/NewWebsite/atwork/workandconf/2006workshop/program.htm

[47] F. arneodo et al., (The ICARUS-Milano collaboration), eprint:physics/0609205, submitted to Physical Review D.

[48] C. Anderson, 'Reconstruction Algorithms", Workshop on Neutrino Physics with Liquid Argon TPC 
Detectors, http://www-lartpc.fnal.gov/NewWebsite/atwork/workandconf/2006workshop/program.htm

[49] R. J. Wilkes, arXiv:hep-ex/0507097; C. K. Jung, AIP Conf. Proc. 533, 29 (2000) [arXiv:hepex/0005046]. Also see talk by Chang Kee Jung to the NuSAG meeting on May 20, 2006. http://www.hep.net/nusag_pub/May2006/UNO_Jung_NuSAG06.pdf

[50] 'Proposal for an Experimental Program in Neutrino Physics and Proton Decay in the Homestake Laboratory," Collaboration: BNL, Brown University, UC/Berkeley, LBNL, University of Pennsylvania, Princeton University, UCLA, University of Wisconsin, University of Kansas, University of Colorado. July 12, 2006. BNL-76798-2006-IR. a modified copy without some figures is at hep-ex/0608023

[51] Homestake PAC report http://nwg.phy.bnl.gov/ diwan/nwg/fnal-bnl/docs/Homestake_PAC_Report_Final_5_12_2006.pdf

[52] Talk by Chris Laughton at the June 27, 28 meeting. Technical note in preparation. http://nwg.phy.bnl.gov/ diwan/nwg/fnal-bnl/june27-28/talks/Geo-Construct1.ppt

[53] INITIAL STABILITY STUDY OF LARGE OPENINGS FOR THE NATIONAL UNDERGROUND SCIENCE LABORATORY AT THE HOMESTAKE MINE, LEAD, SD Doug Tesarik, Jeff Johnson, Karl Zipf, Jr., Kenneth Lande. NARMS - TAC 2002: Mining and Tunnelling Innovation and Opportunity, Vol. 1, pp 157-163 R. Hammah et al Eds.,Toronto, University of Toronto Press

[54] S. Amerio et al. [ICARUS Collaboration], 'Design, construction and tests of the ICARUS T600 detector," Nucl. Instrum. Meth. A 527, 329 (2004).

[55] D. Cline, 'LOI for a Study of a LANNDD of 100kTon at Homestake DUSEL", submitted to the Homestake PAC, 2006.

[56] D. Finley, B. Fleming, S. Pordes, LARTPC-DOC-218, Oct 26, 2006

[57] D. Finley et al., "Work at FNAL to acheive long electron drift time in Argon". Writeup for the FNAL/BNL Long Baseline Study.

[58] S. Pordes, Argon Purging writeup for the FNAL/BNL Long Baseline Study.

[59] D. Jensen, Long Wires technical note for the FNAL/BNL Long Baseline Study

[60] H. Jostlein, Cellular design technical note for the FNAL/BNL Long Baseline study

[61] D. Gerstle and S. Pordes, “Cosmic Ray Rates on a Surface Liquid Argon TPC". http://lartpcdocdb.fnal.gov/cgi-bin/ShowDocument?docid=160

[62] C. Bromberg, 'Deep Underground Cryostat for a LArTPC'. Writeup for the FNAL/BNL Long Baseline Study

[63] L. J. Hall, H. Murayama, M. Papucci and G. Perez, arXiv:hep-ph/0607109.

[64] John F. Beacom, Mark R. Vagins, Phys. Rev. Lett. 932004

[65] Hank Sobel, Presentation at the International Workshop on a Far Detector in Korea, Nov. 18, 2005. http://nwg.phy.bnl.gov/ diwan/nwg/fnal-bnl/docs/Detector_Depth_Issues.pdf 
[66] Physics potential of the Fermilab NuMI beamline. Olga Mena, Stephen J. Parke (Fermilab) . FERMILAB-PUB-05-196-T, May 2005. 22pp. Published in Phys.Rev.D72:053003,2005 e-Print Archive: hep-ph/0505202

[67] 'Precision physics with a wide band super neutrino beam," V. Barger, M. Dierckxsens, M. Diwan, P. Huber, C. Lewis, D. Marfatia, B. Viren, Phys. Rev. D74 (2006) 073004 hep-ph/0607177, BNL-767972006-JA pdf.

[68] 'Which long-baseline neutrino experiments," V. Barger, P. Huber, D. Marfatia, W. Winter, hep$\mathrm{ph} / 0703029$.

[69] M. Ishitsuka [Super-Kamiokande Collaboration], arXiv:hep-ex/0406076.

[70] K. Eguchi et al. [KamLAND Collaboration], Phys. Rev. Lett. 90, 021802 (2003) [arXiv:hepex/0212021]; T. Araki et al., arXiv:hep-ex/0406035.

[71] M. Apollonio et al. [CHOOZ Collaboration] Eur. Phys. J. C 27, 331 (2003) [arXiv:hep-ex/0301017].

[72] Y. Itow et al., arXiv:hep-ex/0106019.

[73] NOvA Proposal to Build a 30 Kiloton Off-Axis Detector to Study Neutrino Oscillations in the Fermilab NuMI Beamline D. S. Ayres et al. [NOvA Collaboration], arXiv:hep-ex/0503053.

[74] D. Beavis et al., arXiv:hep-ex/0205040;

[75] M. V. Diwan et al., arXiv:hep-ex/0306053.

[76] S. A. Kahn and M. Diwan, PAC-2005-RPPT059 Prepared for Particle Accelerator Conference (PAC 05), Knoxville, Tennessee, 16-20 May 2005.

[77] M. H. Ahn [K2K Collaboration], arXiv:hep-ex/0606032.

[78] M. Diwan et al., arXiv:hep-ex/0211001.

[79] S. Eidelman et al. [Particle Data Group], Phys. Lett. B 592, 1 (2004).

[80] P. Huber, M. Lindner and W. Winter, Nucl. Phys. B 645, 3 (2002) [arXiv:hep-ph/0204352].

[81] P. Huber, M. Lindner, M. Rolinec and W. Winter, Phys. Rev. D 73, 053002 (2006) [arXiv:hep$\mathrm{ph} / 0506237]$.

[82] P. Huber, M. Lindner and W. Winter, 'Simulation of long-baseline neutrino oscillation experiments with Comput. Phys. Commun. 167, 195 (2005) [arXiv:hep-ph/0407333].

[83] A.A. Anguilar-Arevalo et al., LA-UR-07-2246, hep-ex/0704.1500v2

[84] L. Borodovsky et al., Phys. Rev. Lett. 68:274-277, 1992.

[85] These are the sensitivity limits expected from the NOvA experiment only, before combination with T2K. Analysis of combining with T2K has been performed elsewhere [66] 